\title{
What is it about Organized Sports? Understanding the Associations between Experiences in Youth Sport and Positive Youth Development
}

\author{
by \\ Katy Hill MacEachern
}

A thesis submitted to the Faculty of Graduate and Postdoctoral Affairs in partial fulfillment of the requirements for the degree of

Doctor of Philosophy

in

Psychology

Carleton University

Ottawa, Canada

(C)2021 Kate Hill MacEachern 


\begin{abstract}
Research indicates that participation in sport is associated with positive youth development (PYD), yet little is known about the mechanisms within sport that foster these positive outcomes. Existing measures of PYD in sport that are focused on outcomes, represent a mix of causal and outcome factors, and/or are missing potentially valuable experiences. Using the Sport-Based Model of Positive Youth Development (Holt et al., 2017) as a framework, this thesis sought to develop and test a measure of youth experience in sport. Three studies were conducted to achieve this objective. In Study One, $N=9$ individuals (aged 13 to 38) participated in interviews about their experiences playing organized youth sports. Using findings from these interviews, literature, and expert review, a three-factor measure of youth experience in sport was developed to assess experiences of goal setting, adversity, and skill development. The factor structure of the Experience in Sport Scale for Youth (ESSY) was then evaluated using exploratory factor analysis in Study Two. With a sample of $N=334$ adolescents $\left(M_{\text {age }}=17.36\right)$, a 19 -item, threefactor model was retained. In Study Three, experience in sport, as assessed by the ESSY, was incorporated into the Sport-Based Model of PYD and tested as a mediating and a moderating factor in the association between PYD climate and PYD outcomes. A total of $N=453$ undergraduate students $\left(M_{\text {age }}=18.46\right)$ completed measures of coach relationships, peer relationships, experiences in sport, and the 5 Cs of PYD. The overall model failed to converge. However, results indicated a significant association between goal setting and PYD outcomes of competence, confidence, character, caring, and connection. Furthermore, skill development was significantly associated with character and caring. Contrary to expectations, adversity was not significantly associated with any of the PYD outcomes. Implications for PYD research and future directions are discussed.
\end{abstract}




\section{Acknowledgements}

There were many days where it felt like this document would never be finished.

Fortunately, I have had some pretty amazing people helping me along the way who pushed me when I felt stuck, carried me when I felt worn out, and cleared the track when I gained steam. I am grateful to anyone who took the time to offer me advice and guidance during my graduate work and there are far more thanks to give than I can acknowledge here.

First and foremost, I would like to thank my committee members, Dr. Katie Gunnell and Dr. Andrea Howard, for their guidance, expertise, and support in developing this thesis. A tremendous thank you to my supervisor, Dr. Anne Bowker, for giving me the opportunity to achieve this goal of mine. You supported me through my master's thesis, through two maternity absences, and through this journey and I have always felt that you had my back. I am particularly grateful for your support in this past year. With all the uncertainty and changing circumstances, I cannot thank you enough for being so understanding, so encouraging, and so positive during the past 13 months.

Thank you to my lab mates through the years. You were my sounding boards and my cheerleaders, and I am so grateful to have been surrounded by so many supportive people. Special shout-outs go to Marisa, Lisa, Belinda, Jen, and, of course, Cecilia, for their willingness to answer my (many) questions and to laugh lots. To my grad-school-bestie, Megan Lamb, you have been my rock for this $\mathrm{PhD}$ journey, and I am eternally grateful to the force that brought us together. I miss our carpooling, days together in the lab, our lunches at Joey's, and so many other things but I cannot wait to spend summer days in Nova Scotia with our families.

I would not be here without the love, support, and encouragement from my friends and family. Thanks for always asking how things were going, even if you were only feigning interest 
in my grad school problems. That said, I am happy that I will not have to answer the question, "when do you think you'll be done" again.

A tremendous, gigantic, all-consuming thank you to my mom and my brothers. Mom, you always believed in me and encouraged me to do anything. You are the most amazing rolemodel, and I am forever grateful for your unfaltering belief in me. To my husband, Ty Ty, I cannot thank you enough for supporting me in this endeavor. It was my goal and you were willing to make it our goal despite the sacrifices. Thank you for the encouragement when I was feeling low, thank you for the patience when I was feeling stressed, thank you for the understanding when I needed the time to work, and thank you for understanding that you will have to call me "doctor" from now. To my little babies, Harper and Leo, I love you more than words. I am grateful that grad school gave me the flexibility to be with you and watch you grow into the little humans you are now. It was not always easy, but I would not change a minute of it.

A final acknowledgement to the people who were not here to see me in this journey but who have taught me so much and who would have been so proud of my accomplishments. My grandmothers, Mary Prescott Curry and Joyce Hill, were incredible, strong women. My grandfather, Robert Hill, was the kindest man who loved his family with his whole heart. And, my dad, Stephen Hill, who had so much fun in life. 


\section{Table of Contents}

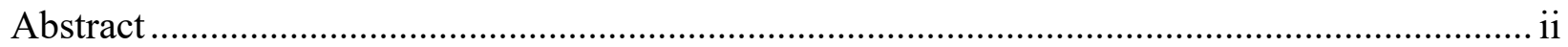

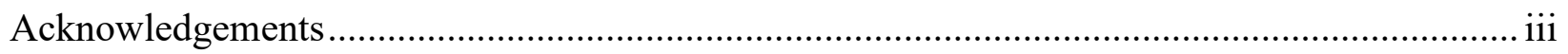

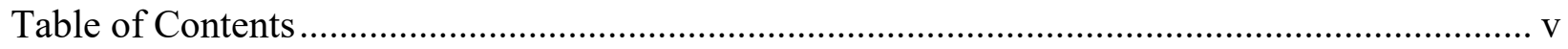

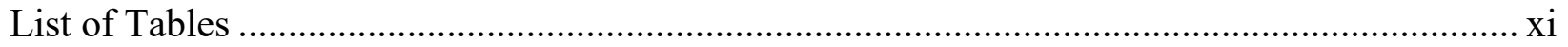

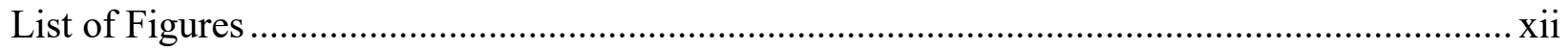

List of Appendices ..........................................................................................................

What is it about Organized Sports? Understanding the Associations between Experiences in

Youth Sport and Positive Youth Development................................................................ 1

Physiological and Psychological Benefits of Physical Activity and Sport ...................................... 3

Physiological Benefits of Physical Activity........................................................................... 4

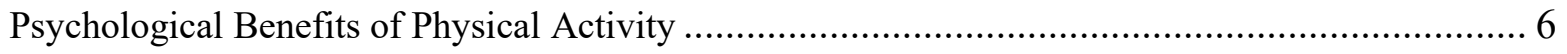

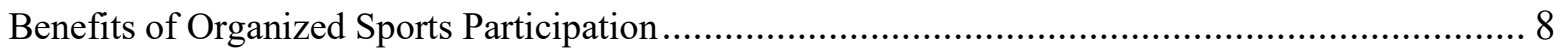

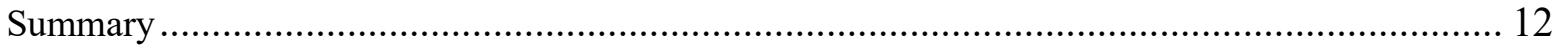

Bronfenbrenner's Theories of Development .................................................................. 13

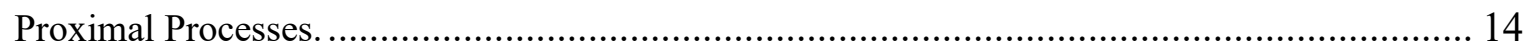

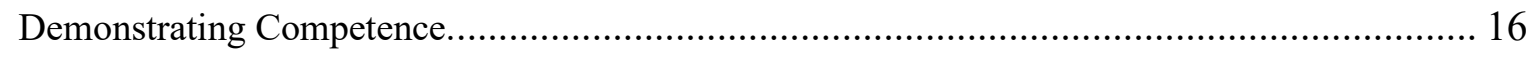

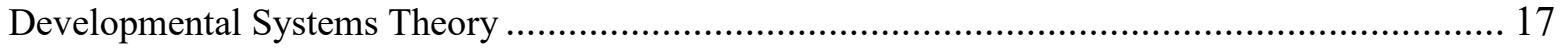

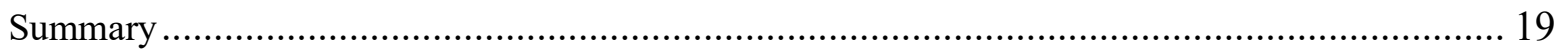

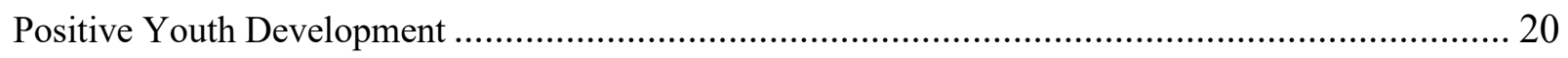


Conceptualizations of Positive Youth Development

The 5 Cs Model.

Initiative, Motivation, and the Youth Experience Scale. 26

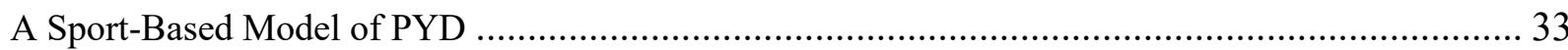

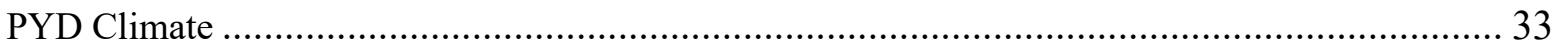

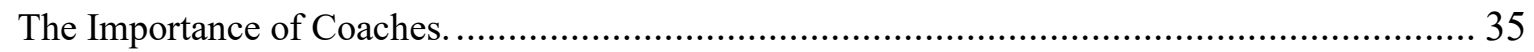

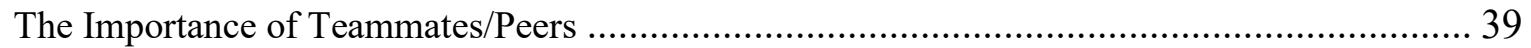

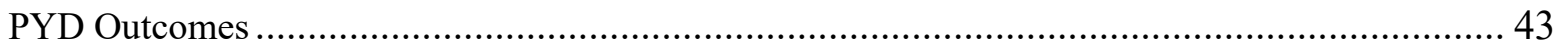

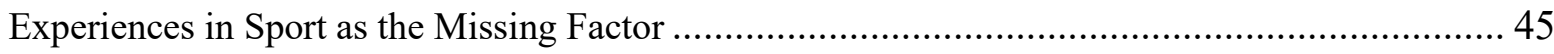

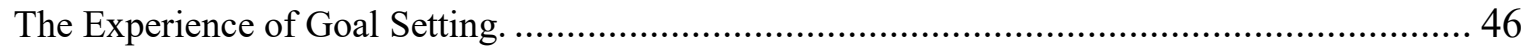

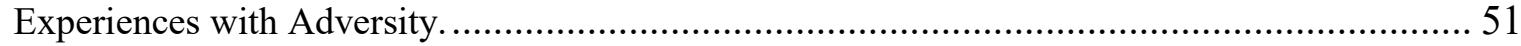

Experiences with Skill Development......................................................................... 53

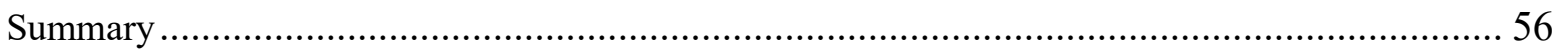

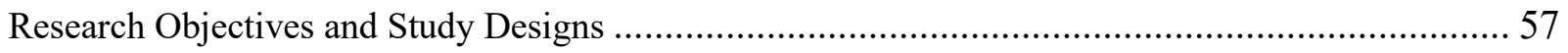

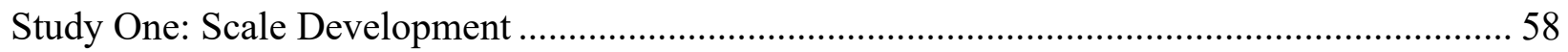

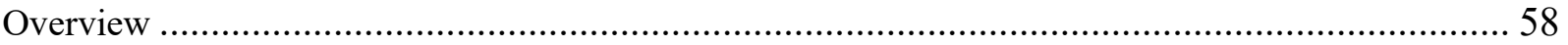

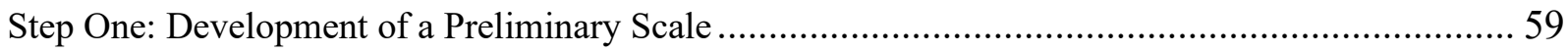

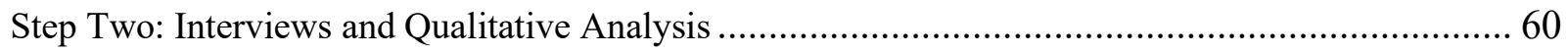

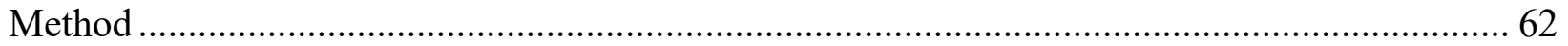

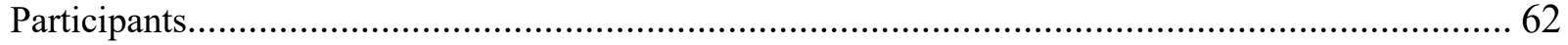




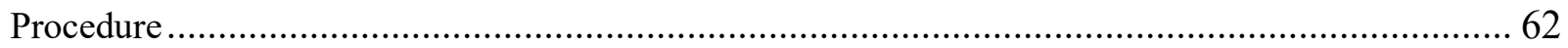

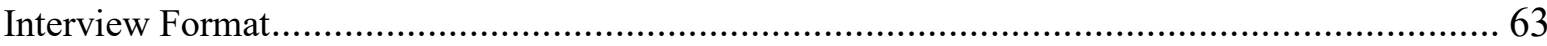

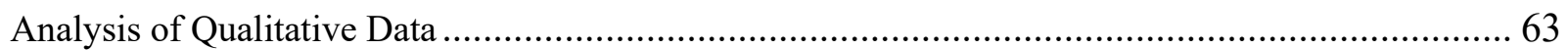

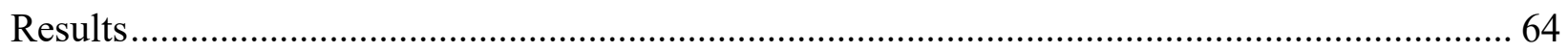

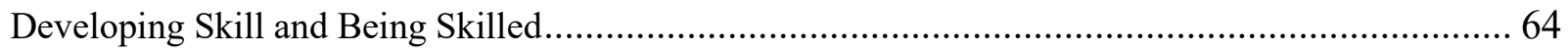

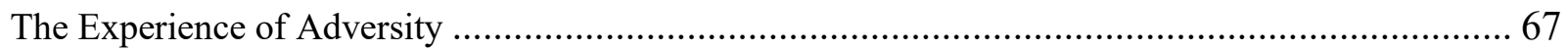

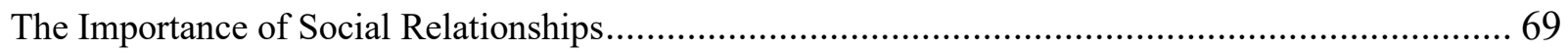

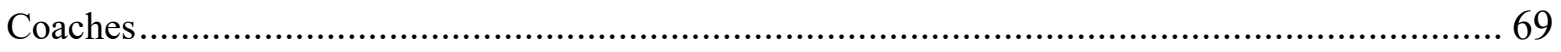

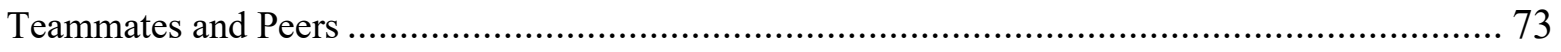

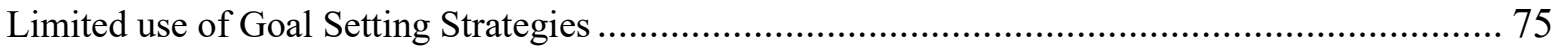

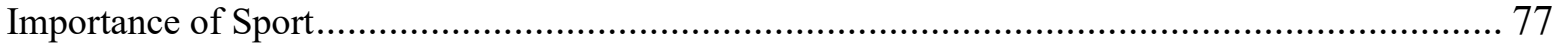

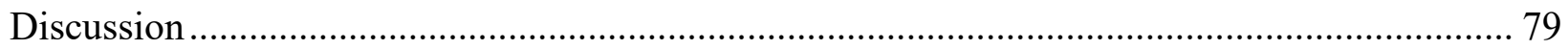

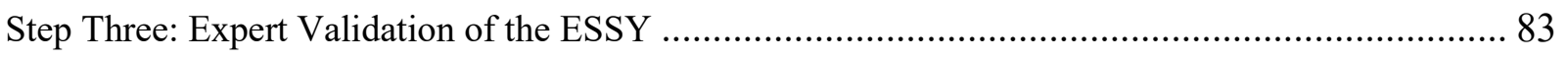

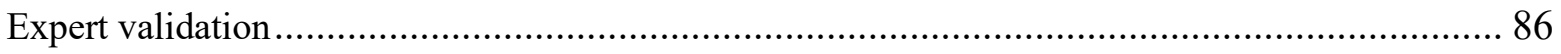

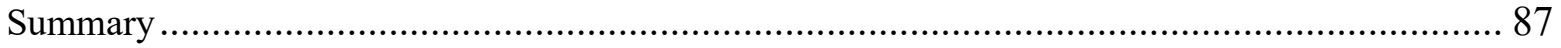

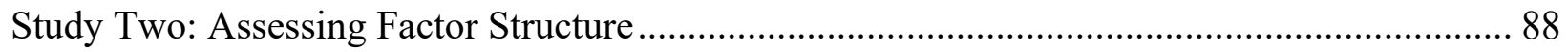

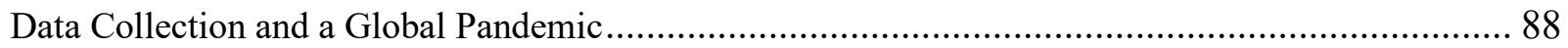

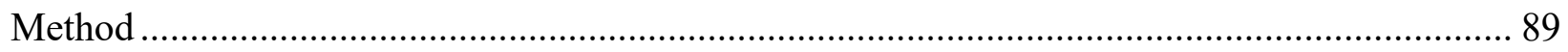

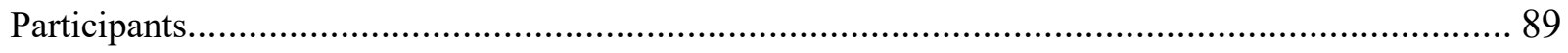

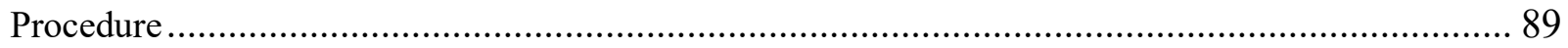


Measures

Results.

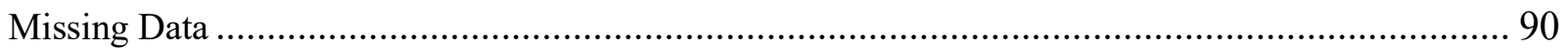

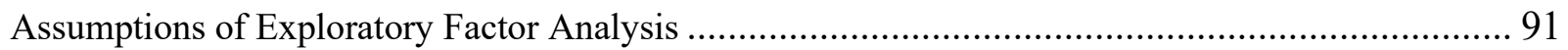

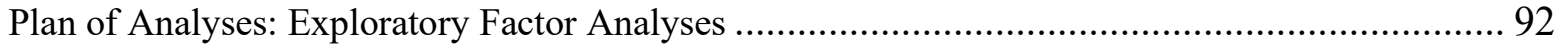

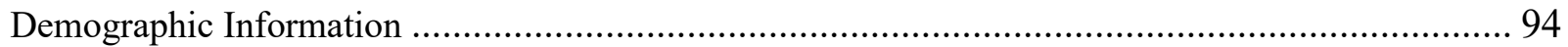

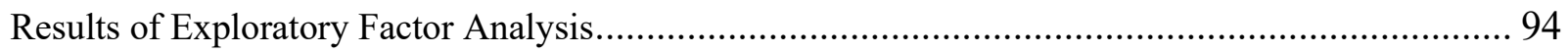

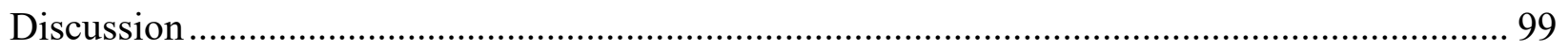

Study Three: Testing the Sport-Based Model of PYD .................................................. 100

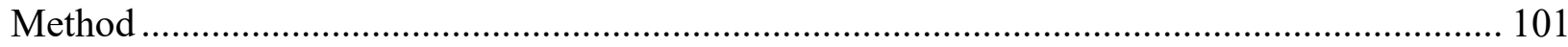

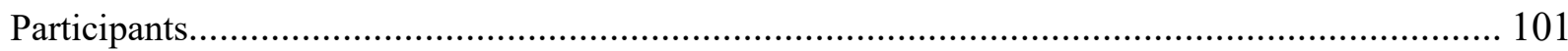

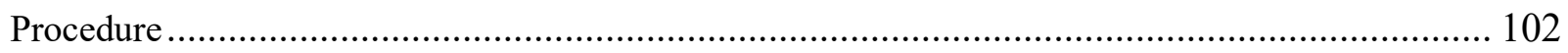

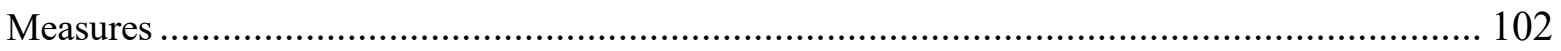

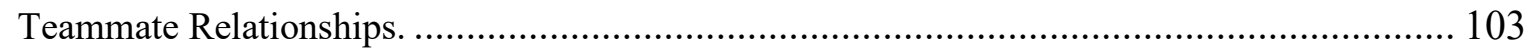

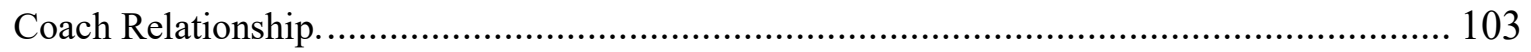

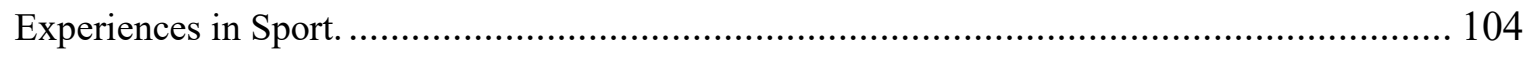

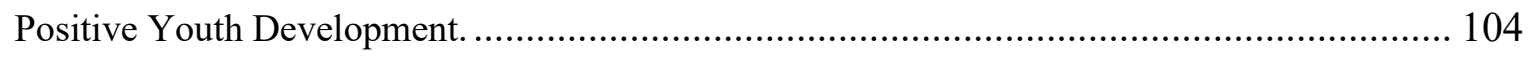

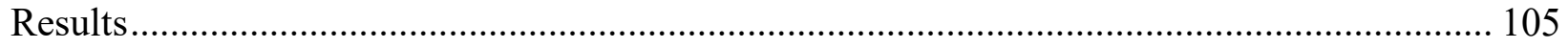

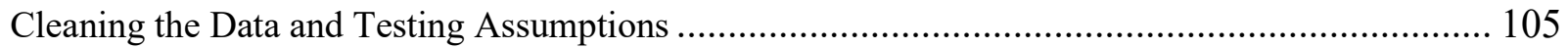




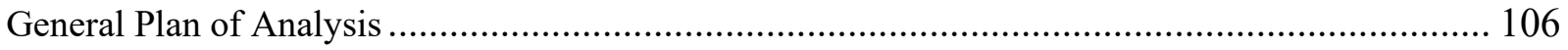

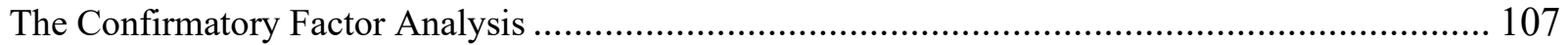

Results of Confirmatory Factor Analysis................................................................... 109

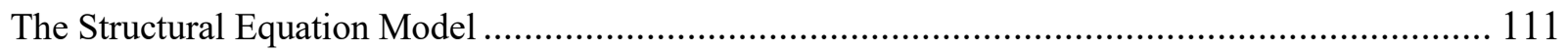

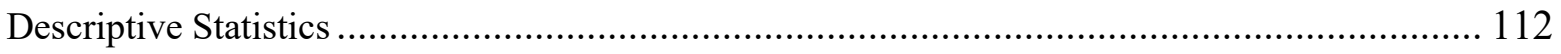

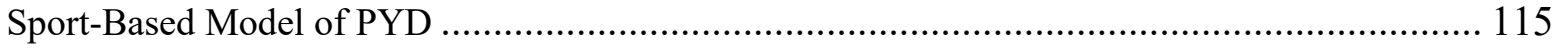

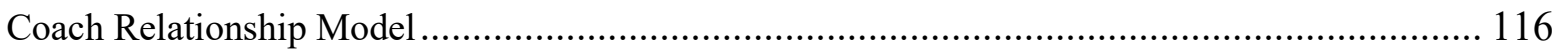

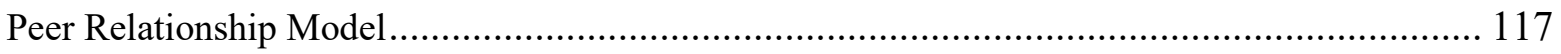

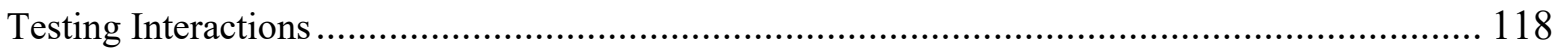

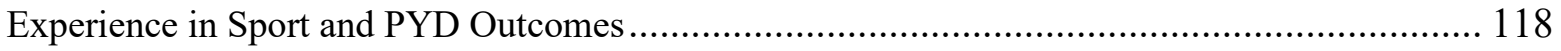

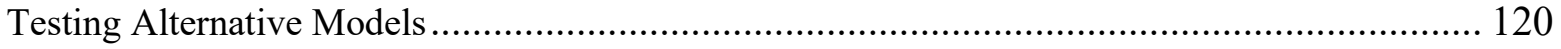

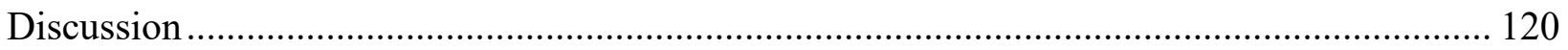

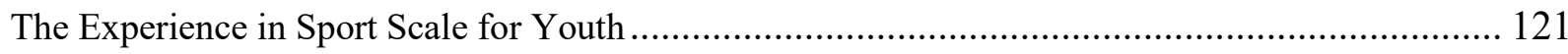

Sport-Based Model of PYD: Goal Setting, Skill Development and PYD .................................. 123

Development Over Time and Taking a Person-Centered Approach ........................................ 125

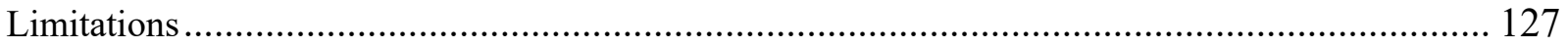

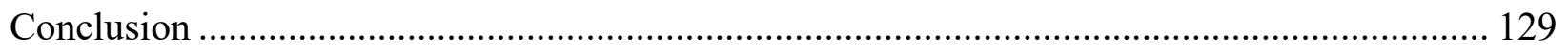

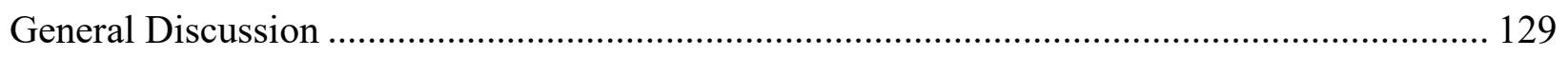

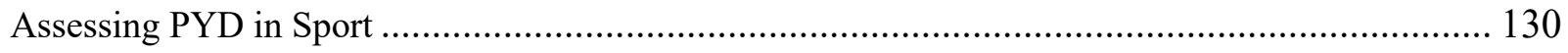

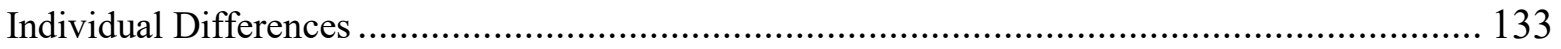


Systems at Play in the Sport Environment ........................................................................... 135

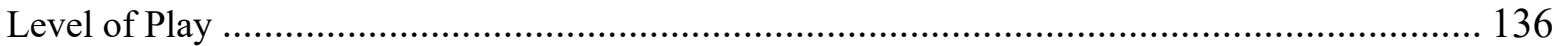

Coaching, Coach Quality, and Individual Preferences .......................................................... 137

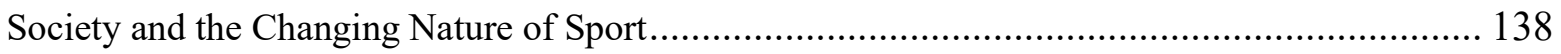

An Interactive Model of PYD in Sport .............................................................................. 140

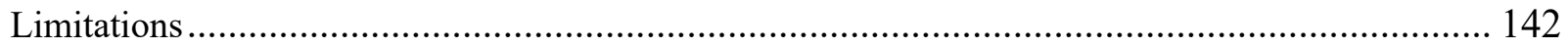

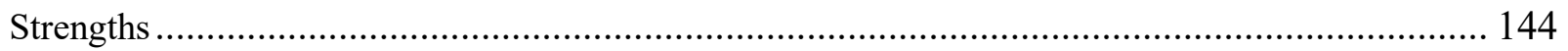

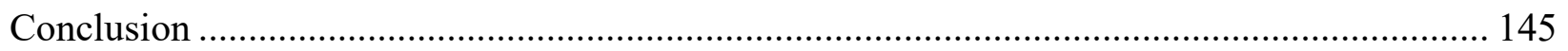

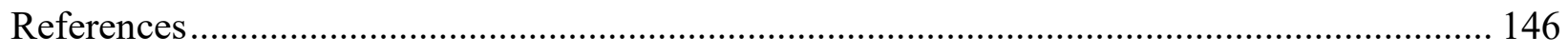




\section{List of Tables}

$\begin{array}{lll}\text { Table Title } & \text { Page }\end{array}$

$1 \quad$ Demographics for Interview Participants 63

2 Preliminary Items for the Experience in Sport Scale for Youth 84

3 Items Representing Sport Climate $\quad 85$

4 Definitions of Constructs for the ESSY 85

$5 \quad$ Factor Loadings from the First Analysis of the ESSY Factor Structure 96

$6 \quad$ Factor Loadings Representing a Three-Factor Solution 97

$7 \quad$ Communalities for ESSY Items 98

$8 \quad$ Standardized Factor Loadings of the ESSY Items 110

$9 \quad$ Fit Indices for Competing Models 111

$10 \quad$ Means and Standard Deviations of Study Variables 113

11 Correlations of Key Study Variables $\quad 114$ 


\section{List of Figures}

Figure Title Page

1 A Sport-Based Model of Positive Youth Development 34

2 Proposed Sport-Based model of Positive Youth Development 58

$3 \quad$ Sport-Based Model of Positive Youth Development to be Tested 101

$4 \quad$ Factor Model of the ESSY 108

$5 \quad$ Structural Equation Model 112

$6 \quad$ Standardized Factor Loadings of the Simplified Model 119

$7 \quad$ Interactional Model of Positive Youth Development Through Sport 141 


\section{List of Appendices}

Appendix Title Page

$\begin{array}{lll}\text { A Initial ESSY Items } & 180\end{array}$

$\begin{array}{lll}\text { B Interview Protocol } & 182\end{array}$

$\begin{array}{lll}\text { C Interview Participant Consent Form } & 184\end{array}$

$\begin{array}{lll}\text { D Parent Consent Form } & 186\end{array}$

E Final Version of Interview Protocol $\quad 188$

$\begin{array}{ll}\text { F } & 190\end{array}$

G SONA Recruitment Poster $\quad 191$

H SONA Participant Consent Form $\quad 192$

I Higher School Student Parent Information Letter 194

J High School Student Consent Form 195

$\begin{array}{lll}\text { K } & \text { Debriefing Form } & 197\end{array}$

L Background Information Questionnaire 199

M Experience in Sport Scale for Youth 205

N Peer Motivational Climate Scale 207

F Coach Motivational Climate Scale $\quad 209$

G Short Form of the 5 Cs of Positive Youth Development 211 

What is it about Organized Sports? Understanding the Associations between Experiences in Youth Sport and Positive Youth Development

Organized sports are an integral part of Canadian society, particularly for youth. Approximately $77 \%$ of Canadian youth aged 5 to 19 and just over $60 \%$ of Canadian youth aged 15 to 19 participate in an organized sport (Canadian Fitness and Lifestyle Research Institute, 2019). Sport represents a unique and valuable environment for youth and there are a multitude of physiological and psychological benefits to participation. From a more general perspective, physical activity is associated with physical health, including cardiovascular health (Warburton et al., 2006), bone density and strength (Vicente-Rodriguez et al., 2005), and the maintenance of a healthy weight (Goldberg \& King, 2007). People who engage specifically in sports are also happier (Rasciute \& Downward, 2010), report higher self-esteem (e.g., Adachi \& Willoughby, 2014), lower depressive symptoms (e.g., Jewett et al., 2014)), and have a more positive body image (Ferron et al., 1999). These represent a number of key benefits to youth.

With these benefits comes the opportunity to enhance youth developmental outcomes through participation in sport. Positive youth development (PYD) is a strengthbased approach to adolescent development that views youth involvement in extracurricular activities, such as sport, as an opportunity to enhance developmental outcomes (Lerner et al., 2014). PYD is rooted in developmental systems theory and the ideas laid out by Bronfenbrenner whereby interactions between a youth and his/her environment influence development. The sport context represents a valuable environment where interactions take place between coaches, teammates, and opponents, and where youth learn new skills, face challenges, set goals, and are exposed to several different 
experiences.

A few different conceptualizations have been proposed to help explain and test PYD in a sport setting. The most well-known conceptualization is the $5 \mathrm{C}$ approach that lists PYD as the development of Caring, Character, Competence, Confidence, and Connection (J. Eccles \& Gootman, 2002). This is an outcome-based approach to PYD that does not assess experiential factors (i.e., how does sport influence PYD). In contrast, Larson offers an approach centered on initiative and motivation. The theory behind this approach is much more process based and attempts to explain how experiences in sport might influence outcomes. However, the scale developed to assess PYD in this manner has a mix of process and outcome constructs. Finally, Holt et al., (2017) has proposed a sport-based model of PYD. This conceptualization does not suggest a manner to measure PYD in sport but offers a structure to be used to study PYD in sport. This includes looking at important social relationships, such as coaches and teammates. Ultimately, at present, there is no way to assess developmental experiences in sport and determine how sport promotes PYD outcomes for youth. This is a large gap in the knowledge base.

There were two overarching purposes for this thesis. One was to develop a measure of adolescents' experiences in sport that was rooted in developmental and PYD theory. With over half of the adolescent population participating in organized sport, there was a specific need to understand how the sport context relates to PYD outcomes and what sport-specific developmental experiences were important to fostering these outcomes. Another aim of this study was to test the implicit pathway in the sport-based model proposed by Holt et al., (2017), incorporating an experiential factor to the association between PYD climate and PYD outcomes. This model has not yet been 
empirically tested.

To achieve the objectives, three studies were conducted. Each of the three studies represents an important part of scale development and helps ensure a valid, reliable, and meaningful measure. Study One incorporated information from the literature review for the development of an initial set of items and constructs. Interviews were then conducted to assess the initial items and to investigate if additional constructs or items were needed. Subject-matter experts were consulted to assess the content validity of the items. This resulted in a preliminary measure, the Experience in Sport Scale for Youth (ESSY) that was subjected to exploratory factor analyses (EFA) in Study Two. The EFA supported a 19-item, three-factor measure assessing goal setting, skill development, and adversity.

Study Three confirmed the factor structure of the ESSY and tested an adapted sport-based model of PYD using structural equation modeling. Experiences in sport, as measured by the ESSY, were tested as mediating and moderating factors in the association between PYD climate (coaches and peers) and PYD outcomes (5 Cs) variables. Hypotheses were only partially supported but did show the experience of goal setting to be associated with each of the five Cs of PYD. The experience of skill development was also strongly associated with character and caring. Results are discussed in terms of future directions for PYD research and how considerations of methodology and study design may provide findings that further elucidate the value of experiences in sport to positive developmental outcomes.

\section{Physiological and Psychological Benefits of Physical Activity and Sport}

There are many positive associations between physical activity and health. These include both physiological and psychological outcomes. Physical activity includes 
activities in which the body is moving, and the individual is exerting energy (Canadian Society for Exercise Physiology, 2012). Unlike sport, these activities can be unstructured or structured and the research focus tends to be more on the time and intensity of this energy exertion. Physical activity is a necessary component of sport participation, but it is not necessary to be engaged in a sport to be physically active. As such, an overview of the benefits associated with physical activity will be discussed first followed by a discussion of the benefits associated, more specifically, with sport participation.

\section{Physiological Benefits of Physical Activity.}

Participation in physical activity has, for the past few decades, been espoused as an important part of a healthy lifestyle for both children and adults. Multiple researchers and government agencies recommend the incorporation of at least 60 minutes of physical activity per day for children and adolescents (e.g., Canadian Society for Exercise Physiology, 2012; Janssen \& LeBlanc, 2010; Strong et al., 2005). Research shows significant physiological benefits ranging from improved skeletal health (e.g., Linden et al., 2006; Vicente-Rodriguez et al., 2005) to cardiovascular health (Blair et al., 2001) to the maintenance of a healthy weight (Franzini et al., 2009; Hills et al., 2007; Warburton et al., 2006). In addition, participating in regular physical activity may reduce the likelihood of developing some types of cancers, such as colon and breast cancer (Blair et al., 2001; Warburton et al., 2006) and may even prevent premature death (Warburton et al., 2006).

Physical activity is also an effective method to enhance bone health among children and adolescents (see Vicente-Rodriguez, 2006 for a review). In fact, because bones go through growth spurts and see an increase in mineral deposits during childhood 
and adolescence, exercise and sport involvement may be especially important during this period (Hills et al., 2007). In line with this research, guidelines also recommend the incorporation of bone strengthening exercises for youth along with 60 minutes of moderate physical activity each day (Canadian Guidelines for Physical Activity, 2012; Janssen \& Le Blanc, 2010).

Perhaps the most widely cited physiological benefit of physical activity is the reduction of obesity and the maintenance of a healthy weight. Physical activity has been touted as an essential way to enhance physical fitness for youth and reduce the likelihood of obesity with research consistently showing a significant relation between increased physical activity and a decrease in obesity and being overweight (Franzini et al, 2009; Hills et al., 2007; Warburton et al., 2006). In a study of 7081 children and adolescents (6 to 18 years old), both boys and girls who were the least active reported more body mass index (BMI) levels in the 95th percentile and above (Katzmarzyk et al., 2007). This supports findings of an intervention study incorporating physical activity into an obesity treatment plan. After 16 weeks, results showed that an increase in physical activity was associated with a significant decrease in BMI for a group of male adolescents with obesity (Kim \& Lee, 2009).

Taken together, there is a great deal of evidence demonstrating the physiological benefits of physical activity. There is some evidence to suggest that these benefits may be especially important for children and adolescents as it can lay the groundwork for future health outcomes as an adult. It is worth noting that, along with the physical activity recommendation, at least one paper has stated that involvement in physical activities should be both enjoyable and developmentally appropriate for youth (Strong et al., 2005). 
As the research would suggest, it is essential that children develop an interest in physical activity so that they can continue with it and reap the many physiological rewards.

\section{Psychological Benefits of Physical Activity}

There is an abundance of research showing a positive association between physical activity and positive psychological outcomes. Perhaps the most widely examined and consistently discussed associations are between physical activity and depressive symptoms and physical activity and anxiety. Both clinical and non-clinical populations have shown decreases in depressive symptoms with the increase in physical activity (Emerson \& Williams, 2015). Some have even argued that physical activity can be used as a method to help treat major depressive disorder (Dunn et al., 2005). In a particularly large study of high school students in the United States $(N=13633)$, Sibold et al., (2015), found that physically active students were less likely to report feeling sad or to experience suicidal ideation.

Similar to depression, those who are physically active report fewer symptoms related to anxiety as compared to their inactive counterparts (De Mello et al., 2013; Jewett et al., 2014; Lindwall et al., 2014; Strohle et al., 2007). Furthermore, changes in physical activity levels have shown simultaneous decreases in anxiety symptoms (Lindwall et al., 2014). Multiple reviews and meta-analyses have found a small but significant relation between regular physical activity and the reduction in anxiety and symptoms of anxiety (e.g., Biddle \& Asare, 2011; Emerson \& Williams, 2015; Moksnes et al., 2010; Rebar et al., 2015; Strohle et al., 2007).

Physical activity appears to play an important role in the development of selfesteem and global self-worth for adolescents. Studies have shown a positive association 
between physical activity and self-esteem. When looking at these two variables on their own, it would appear that physical activity enhances self-esteem. However, when other variables are added into the mix, the relation becomes more nuanced. Bowker (2006), looked at the role of physical competence or physical self-esteem and found this to mediate the association between physical activity and self-esteem. This would seemingly make a lot of sense as successfully using one's body in a functional way enhances perception of one's body and, as the body is a key component of who an individual is, this would lead to a stronger self-concept. To put it more succinctly, physical activity leads to higher physical self-concept and that leads to higher self-esteem.

One more recent and interesting finding is the apparent association between physical activity and cognitive functioning in children and adolescents. Researchers have found that an increase in physical activity corresponds to enhanced cognitive functioning, such as problem solving (Chaddock-Heyman et al., 2013; Hillman et al., 2008; Tomporowski et al., 2008). Using fMRI technology, several studies have even shown differential and enhanced brain activation in physically active youth as compared to those who are physically inactive (Chaddock et al., 2012; Chaddock-Heyman et al., 2013; Davis et al., 2011). Esteban-Cornejo et al., (2014) conducted a study with 1662 adolescents and found that, overall, vigorous physical activity was positively related to verbal, numeric, and reasoning variables. Furthermore, individuals who were involved in an organized activity scored better than those who did not participate in an organized physical activity. Finally, individuals who were involved in more than one activity also scored better than those only involved in one activity. This is an interesting result as it not only shows the value of physical activity but also points to a potential additive effect 
where an increase in the type of physical activity (i.e., playing more than one sport) may ameliorate the effect of being physically active on brain development.

\section{Benefits of Organized Sports Participation}

As the purpose of this research is to examine the factors associated with positive developmental outcomes in a sport environment, it is important to provide an overview of some of the benefits associated, specifically, with participation in organized sport. Although physical activity is a necessary component of sport (i.e., neuromuscular skills are identified as a salient feature of sport; Canada and Canadian Heritage, 2014), there are specific aspects to a sport setting that make it a unique context and may contribute to positive developmental outcomes for youth above and beyond those associated with physical activity.

Sports are, generally, more structured and organized with a specific environment in which the sport is carried out (a hockey rink, golf course, swimming pool, etc.) and organized times and specific places in which the sport takes place. There is a competition aspect between two or more individuals and there are specific rules and formalities associated with participation (Canada \& Canadian Heritage, 2014). Sports also allow youth the opportunity to develop relationships with adults and other peers and experience things like goal setting and teamwork. Overall, sports offer youth greater opportunity and additional benefits to physical activity. The Cambridge Dictionary has defined sport as, "a game, competition, or activity needing physical effort and skill that is played or done according to rules, for enjoyment and/or as a job" (Cambridge Dictionary, 2021). For the purpose of this thesis and consistent with Canadian Heritage and Cambridge Dictionary 
definitions, organized sport was considered any team or individual physical activity with organized times for practices/games and competitions against others.

Similar to the physical activity research, athletes have reported feeling healthier, having a stronger body image (Ferron et al., 1999), lower depressive symptoms and stress (Jewett et al., 2014). From an academic standpoint, both male and female adolescents involved in sports report higher GPAs as compared to those not involved in sports (Fox et al., 2010; Stephens \& Schaben, 2002). Youth involved in sports are also less likely to engage in risky behaviour such as drug and alcohol use (Ferron et al., 1999) and are even less likely to attempt suicide (Taliaferro et al., 2011). These are important benefits for adolescents, particularly at a time when adolescent mental health issues are prevalent and receiving added attention.

From a more general perspective, sport participation appears to positively influence satisfaction and mental health indices. Snyder et al., (2010), compared a group of athletes to non-athletes and found that those who participated in sports reported higher scores on measures assessing physical and mental health. In addition, Eime et al., (2013), reported higher self-esteem and lower depressive symptoms and improved psycho-social health with those who participated in sports. The authors also suggested that team sport involvement may be even more beneficial as studies showed higher scores on psychosocial health indices (e.g., loneliness, life satisfaction, self-esteem) for participants in team sports. Eime et al., concluded by stating that participation in sport, and not just physical activity, should be encouraged for children and adolescents. Backing up this contention, Badura et al., (2015), found that adolescents who participated only in sports, compared to adolescents who participated in various extra-curricular activities (e.g., art, 
drama), reported the most positive development outcomes, as indicated by higher life satisfaction scores, better sleep, decreased nervousness and less depression.

Studies have examined the role of sports on body image concerns, particularly with female adolescents. According to Abbott and Barber, (2011), participating in organized sport is related to greater body satisfaction compared to those who are active but not in an organized sport. Participants of organized sports (as compared to physical activity) appear to value their body more as a tool and less as an object (Abbott $\&$ Barber, 2011). This would suggest that sport participants are less concerned about what their body looks like and more about how it can work for them. Adding another layer of interest to the association, participating in a sport with an aesthetic component (e.g., dance, figure skating) correlates with more negative body-related outcomes (Carter \& Rudd, 2005; Slater \& Tiggemann, 2006) and is true for both males and females. Such negative outcomes include disordered eating, self-objectification, and a drive for thinness and/or muscularity (Carter \& Rudd, 2005; de Bruin et al., 2007; Krentz \& Warschburger, 2013). Sport, in general, may lead to a more positive body image, but the type of sport and reasons for participating, also appears to play a role.

The sport environment offers many opportunities for youth. These include developing skills, learning to compete, socializing with teammates and competitors, and developing relationships with adults. Chard et al., (2015) conducted a qualitative study asking parents to identify the attributes and benefits they perceived to be associated with youth sport participation. These were parents of youth involved in rep (i.e., competitive) hockey. Some of the attributes included experiences such as the challenge of competition and working within a team environment (Chard et al., 2015). The consequences, or 
positive outcomes, of participating in competitive hockey included the opportunity to learn life lessons, develop skills, show discipline and be accountable for actions, develop a work ethic, and create friendships (Chard et al., 2015). Many of these attributes represent characteristics and skills that would be beneficial to an adolescent in their longterm development.

Just as parents have identified positive attributes associated with the sports environment, adolescents have also articulated various positive experiences that sport has provided them. In a qualitative study, Fraser-Thomas and Côté (2009) asked a group of adolescent swimmers to describe their experiences in the sport of swimming. Athletes described swimming as contributing to things such as work ethic, time management skills, developing resiliency, enhancing communication skills, and contributing to the development of stronger relationships with peers and coaches (Fraser-Thomas \& Côté, 2009). Such outcomes represent a variety of important skills that could have implications for development. Being provided with the opportunity to develop resiliency, for example, may help an athlete in future stressful situations successfully manage their emotional and physical response. This could be sport-related stress or translate into non-sport domains, such as stress related to exams or conflict with parents.

Consistent with such qualitative findings, Larson et al., (2006) reported several positive outcomes for adolescents participating in sports compared to other extracurricular activities (e.g., performance art, faith-based groups). Specifically, participating in sport was associated with higher initiative, emotional regulation, and teamwork experience scores (Larson et al., 2006). Larson et al., suggest that sport may provide a context for adolescents to develop these skills. In other words, through 
experiences consistent with the sport environment, adolescents can develop skills that may enhance their development.

It is important to note that not all experiences in sport are positive. During discussions with youth swimmers, Fraser-Thomas and Côté (2009), reported themes related to negative experiences with coaches, peers, parents, and a challenging psychological environment (i.e., stress and negative self-perceptions). However, there is little research assessing how these negative experiences might influence developmental outcomes.

\section{Summary}

Taken together, the research suggests that there is something within the sport context that helps to promote positive developmental outcomes for youth. Although it is evident that sport does contribute to positive developmental outcomes, it is unclear how this happens. This is an important piece of the puzzle that is currently missing from the knowledge base. Understanding how and which mechanisms lead to positive developmental outcomes can help make experiences in sport better and make for more effective developmental contexts.

\section{The Theoretical Foundation}

As demonstrated by the literature, there are multiple benefits for adolescents who participate in physical activity and sport, in particular. Researchers have begun to examine this context using the framework of Positive Youth Development (PYD) theory. This framework provides researchers with important theoretical underpinnings and a way to assess sport as a context for development. Positive youth development is a more recent area of research looking at ways to encourage the positive aspects of adolescent 
development and has provided a framework for much of the research looking at the benefits of sport participation in youth. Although PYD as a specific concept is relatively new, the theories and ideas espoused by it are rooted in development theory (Lerner et al., 2001). Bronfenbrenner's theories on development and developmental systems theory (DST) helped shape the current notion of PYD and provide insight into why and how sport appears to be an important context for positive developmental outcomes. To assess experiences in sport and positive developmental outcomes, it is necessary to first understand how organized sports can act as a context for development.

\section{Bronfenbrenner's Theories of Development}

Bronfenbrenner and his theories on the developing person represent an important stepping-stone in the development of PYD theory. Much of what Bronfenbrenner theorized appears to lay the foundation for the current structure of PYD theory. In addition, much of what Bronfenbrenner talks about can be related to the exploration of PYD in a sport setting. The developmental mechanisms at play can be paralleled in the sport context and may be an important indication of why sport has been linked to important psycho-social outcomes in youth.

Bronfenbrenner and Morris (1998) believed that development occurred through consistent interactions between an individual and his/her external environment. To provide a framework, Bronfenbrenner conceptualized a hierarchical model that outlines a series of systems within which individuals develop commonly known as the ecological theory of human development. It begins with the individual and then extends to the Microsystem, which includes the immediate environment of the individual. These would include such things as school, sports teams, and peer groups. This system is set within the 
Mesosystem, which consists of the associations, and processes that occur between the individual and their environments (Bronfenbrenner, 2005). For example, a child's parents going through a divorce impact a child's academic performance or a mother encourages a child to practice their basketball skills by playing games with her at home resulting in improved performance on the basketball team. The two outer rings of the model are systems that play an indirect, yet, still important, role in development. The Exosystem contains such environments that the individual would not come into contact with directly, such as a parent's place of work, but through their influence on the external factor would influence development (Bronfenbrenner, 1986). The final system, the Macrosystem, represents the broadest category in terms of scope. Macrosystems include cultural norms, governmental policy, and economic factors. Although such things do not have a direct role in development, the societal environment within which a child is raised will undoubtedly play a role.

What is most relevant to the present study and to PYD theory is the idea that context can influence development and the reciprocal associations within the Mesosystem play an important role in development. More specifically, the reciprocal nature of the interactions between the individual and his/her environment and how these interactions may influence development is essential to understanding how youth programming can be structured to enhance or promote positive developmental outcomes within a PYD framework. This is essentially the core of PYD models; the notion that contexts can be important and influential in youth development and can be modeled to produce positive outcomes.

Proximal Processes. Bronfenbrenner emphasizes the importance of interactions 
between an individual and environments, people, objects, and symbols. He further outlined an important feature that should be present for these interactions to be effective in promoting human development. That is, these interactions need to be consistent and maintained over time (Bronfenbrenner \& Morris, 1998). Bronfenbrenner termed these types of interactions proximal processes and considered them to be the driving forces behind development (Bronfenbrenner, 2005). Examples of proximal processes include sport involvement, caretaker and child relationships, and child-teacher interactions.

As stated, these proximal processes need to occur with regularity in order to be effective. Further to that, Bronfenbrenner and Morris (1998) identified three characteristics of effective proximal processes. The first is that activities should become more challenging as time goes on. The second is that there must be a bidirectional influence. And, finally, interactions can be between the individual and objects or symbols. Regarding the final point, it is important to note that any interaction that takes place between the individual and an object or symbol (e.g., child and a stick and puck) must be one where the individual is actively engaged with the object or symbol (Bronfenbrenner \& Morris, 1998).

The sport environment appears to be ready-made for such interactions as the very nature of sport and athletics fit the requirements laid out by Bronfenbrenner. As children progress through their sport(s), skills become more advanced through the acquisition of new skills and the perfecting of basic skills. In addition, competition becomes more challenging both as improvements in skill are made and as children age. Furthermore, interactions in a sport setting are bidirectional among parents, coaches, peers, officials, and even the ball/puck. 
One basic example would be the teaching of a new skill as the interactions between the coach and child would change as the skill is taught, learned, and perfected and then either built upon or the child and coach would move on to another skill. The coach is influencing the development of the skill and the child influences how the skill is being taught (by his/her ability and understanding). Regarding the final characteristic, sport involves the interaction of an individual with an object such as a ball or puck or piece of equipment. By its very nature, this interaction requires that the individual be attentive, engaged, creative, and motivated.

There has been some empirical work taking a bioecological approach to outcomes in sport. Dorsch et al., (2015) looked at a small sample of parents, children, and coaches involved in youth soccer. Through interviews, the authors found that sport provided a new context for family relationships. Parents recognized that there was an expectation of how they were to act in a sport setting and began to act that way (e.g., how they spoke to their child in the sport setting). In addition, as parents became engaged in the sport setting, they recognized the opportunity to use sport to communicate and teach certain life skills (Dorsch et al., 2015). The sport context essentially changed, or enhanced, the relationships between parent and child by offering a different context in which to communicate. This led to the purposeful teaching of specific life skills and, as such, affected the development of the child.

Demonstrating Competence. An expected outcome of youth is the development of competence. This may mean athletic competence, academic competence, social competence, etc., depending on the activity, program, or skill being addressed. Bronfenbrenner considers this in an either/or scenario; you are either competent or not. 
Basically, an adolescent is competent when they are demonstrating the acquisition and continued development of skills in a given area (i.e., intellectual, physical, socioemotional; Bronfenbrenner \& Morris, 1998). This is a concept that can easily be paralleled in the sport context where multiple areas of skill development are possible. Physical skill is the most obvious and salient, but it is also likely that young athletes are learning social skills, emotion regulation skills, resilience, etc. This may vary depending on the level and intensity of play, as well as the type of sport that an individual is involved in.

\section{Developmental Systems Theory}

Bronfenbrenner's conceptualization of development falls within the arena of developmental systems and shares some conceptual ideology with developmental systems theory. Developmental systems theory (DST) is the one most commonly referenced as the basis for the PYD movement and is considered to be the basis for contemporary research in developmental psychology (Lerner et al., 2001). It is a more streamlined and concise theory of human development that considers development to be lifelong (Lerner et al., 2001, 2014). The foundation of DST rests on the idea that there is not a singular answer for what impacts development. Biology is not the sole driving force and neither is environment (as an example) but rather the interaction between multiple systems. Furthermore, rather than focus on the manipulations of an experimental setting to delineate causal effects, a developmental systems approach uses contextual information over time to assess the bidirectional nature of developmental influences (Lerner \& Schmid Callina, 2014).

The crux of DST is reciprocity of interactions among individuals and the multiple 
contexts within which they interact (Ford \& Lerner, 1992; Molenaar et al., 2013). Youth are a product of their interactions within their environment and should be studied as such (Lerner \& Overton, 2008; Mueller et al., 2011). This is stating that, within a given context, variable A is both influencing and being influenced by variable B. Furthermore, this association is being influenced by the various other variables (e.g., biological, temperament, age) that may be present within the context (Chaddock-Heyman et al., 2013; Ford \& Lerner, 1992; Lerner et al., 2001; Lerner \& Schmid Callina, 2014). These interactions are what drive development.

Another key element of DST is the belief that development occurs across the lifespan (Baltes, 1987; Birkel et al., 1989; Ford \& Lerner, 1992). The type and outcome of development may differ based on life stage (e.g., an infant experiencing the loss of a family member will not have the same developmental outcome as an adolescent in a similar situation). However, the idea is that individuals at every stage in life can continue their development. At its core, there is the belief that providing youth with a context to develop will promote change and foster positive developmental outcomes.

As with Bronfenbrenner, developmental systems theory posits that competence is an important developmental outcome. Ford and Lerner (1992) describe a competent person as someone who has developed self-regulation skills. From the developmental systems perspective, this is a vital task for adolescents. Through the development of selfregulation (an action that occurs across the lifespan), it is possible for individuals to become actively involved in decisions and better mold the contexts within which they engage and, ultimately, play an important role in development (Ford \& Lerner, 1992). Self-regulation refers to adaptive, goal-directed behaviour that allows an adolescent to 
observe and reflect on a situation and change behaviours if required (Lerner et al., 2001). Thus, the acquisition of self-regulation is an important asset for adolescents as it is both a beneficial skill and it helps them to influence their own developmental pathways.

An essential aspect of developmental systems research is that the development of the individual be considered from a more holistic approach. Individual characteristics and contextual factors must all be considered. This is an interesting and important perspective as it suggests that context can be manipulated (or optimized) to promote specific outcomes. This is critical to the notion of PYD as it has often been used to inform the creation of programs specifically designed to promote the acquisition of characteristics associated with positive youth development. An example of this is The First Tee program which is designed to teach core values while teaching children the game of golf (First Tee, 2021). Some of these values include honesty, integrity and perseverance.

\section{Summary}

Bronfenbrenner and DST provide a strong theoretical basis for how to assess developmental contexts and positive developmental outcomes. The importance of significant others, how contextual factors can influence experiences and development, the development of competence, interactions among various systems and symbols/objects, and the plasticity of human development are essential components. With each of these key components present in the sport environment, sport appears to be an appropriate context in which to study youth development. Moving from this foundation, the next section will provide an overview of PYD theory and how PYD has been conceptualized in the literature. 


\section{Positive Youth Development}

The PYD movement came about in response to what researchers believed was an overemphasis on the negative outcomes associated with adolescent development. The old adage that adolescence was a period of "storm and stress" was seemingly only representing one side of the developmental story. As the name would suggest, PYD takes a positive approach to adolescent development with the belief that, given the right circumstances and opportunities, adolescents can experience positive developmental outcomes (Lerner et al., 2009). The specific outcomes expected from PYD can vary. Youngblade and Theokas (2006) suggest that an adolescent who has developed positively is someone with a sense of purpose, who is happy, is engaged in meaningful relationships and contributes positively to their community (e.g., family, school, community). Note that PYD is concerned with adolescent development, specifically, and offers a way in which to understand development during this period (Rich, 2003). Adolescence typically refers to the periods between ten and 19 years of age, but recent arguments have been made to extend this developmental period into the early 20s (Sawyer et al., 2018).

As laid out by developmental systems theory, three core concepts related to PYD are the notion that development occurs over time and across the lifespan, that youth possess the ability to change (plasticity), and that there is an exchange between the person and environment that promotes development (Benson, 2006). The expectation is that, given the appropriate context, youth will thrive and develop in positive ways. More specifically, Lerner et al., (2014) state that aligning the strengths of the individual with key contextual factors will lead to positive developmental outcomes. Termed adaptive developmental regulation, it is believed that the more an individual can be matched with 
their environment, the better (Benson et al., 2006).

PYD theory suggests that youth will seek out environments that are best suited to their strengths and that parents, teachers, coaches, etc., can play an important role in encouraging or suppressing such behaviour (Benson et al., 2006). As such, it is important for adolescents to have access to places that are considered safe, activities that present some sort of challenge and individuals within these environments that offer emotional support (Roth \& Brooks-Gunn, 2003). These are the hallmark factors associated with positive developmental settings. Lerner et al., (2009) identify several contexts within a youth's environment that could provide such opportunities. These include neighbourhoods, schools, families, and out-of-school activities. Although the present research is concerned with the role of sport in PYD, developmental contexts that could promote PYD outcomes are numerous. Music lessons, drama clubs, Girl Guides, volunteer activities, and church groups are all considered to be excellent resources for adolescents (Bundick, 2011; Eccles et al., 2003; Forneris et al., 2015).

Depending on the specific PYD framework employed, characteristics and expected outcomes differ. That said, after reviewing the literature, common ground can be found (e.g., Benson et al., 1998; Benson, 2006; Larson et al., 2006; Roth \& BrooksGunn, 2003). Thus, programs or activities hoping to achieve positive developmental outcomes need to do the following: (a) Be structured (e.g.,, have assigned times with specific start and end times), (b) provide the opportunity for supportive relationships between the participant and an adult, and between the participant and his/her peers, (c) have a skill-building component, (d) be a psychologically safe environment (e.g., a welcoming environment where the child feels accepted and valued) that promotes socio- 
emotional growth, and (e) offer the participants opportunities to direct their own behaviour (e.g., provide leadership training). It is easy to see why sport would provide an ideal context given these criteria. Organized sport is quite structured, provides the opportunity for athletes to develop relationships with coaches and teammates, skill development is very important for sport, and the environment is intended to be psychologically safe.

It is worth noting that encouraging positive development does not mean that negative behaviours will be extinguished within an individual (Silbereisen \& Lerner, 2007). The two cannot be considered opposites. Rather, adolescent negative behaviours such as delinquent behaviour or drug use, may diminish with involvement in positive developmental activities (Mannes et al., 2005). When discussing sport involvement, this is an important point to consider, as sport has been associated with some negative behaviour, such as increased consumption of alcohol (Kwan et al., 2014; Partington et al., 2013).

\section{Conceptualizations of Positive Youth Development}

A few researchers have attempted to model PYD and provide a framework for investigating PYD in different contexts. Lerner's 5 Cs are primarily concerned with identifying specific developmental outcomes, while Larson's model is more concerned with PYD experiences within an activity. The following sections will focus on a brief overview of each conceptualization, discuss how PYD is operationalized and measured, and review any empirical studies using the measure.

The 5 Cs Model. The $5 \mathrm{C}$ model of positive youth development is, arguably, the most well-known and cited model in the research literature. PYD is conceptualized as an 
'outcome' with five components. The 5 Cs are Competence, Confidence, Connections, Character, and Compassion. Eccles and Gootman (2002) originally defined these outcomes after the authors conducted a review of the literature on developmental outcomes in adolescence. Roth and Brooks-Gunn (2003) echoed these definitions after reviewing the goals associated with 48 youth programs professing to promote PYD outcomes. The programs included in the study ranged from anti-bullying programs and suicide prevention to the Big Brothers/Big Sisters program (Roth \& Brooks-Gunn, 2003). These definitions provided a much-needed way to operationalize PYD and provide tangible outcome goals for PYD programs. The idea being that programs can be designed in such a way to promote the development of the $5 \mathrm{Cs}$.

The definitions given by Roth and Brooks-Gunn (2003) are as follows: Competence is success in the social, cognitive, and vocational arenas; Confidence consists of self-esteem, identity, and belief in the future; Connections refers to relationships with others and with schools and other institutions; Character includes selfcontrol, positive behaviours, respect for rules and standards, morality, spirituality; Compassion is empathy and identification with others. A sixth $\mathrm{C}$ is occasionally referenced as an additional outcome in that once the 5 Cs have been developed, an adolescent will then be equipped to Contribute in a positive manner to society (Lerner et al., 2005). The 5 Cs model is an outcome-based model of PYD and does not take into consideration the process by which youth develop these 5 Cs.

To measure the $5 \mathrm{Cs}$, Lerner and colleagues used subscales of existing questionnaires within a longitudinal study of adolescent development for the 4-H Club of America. The purpose of the 4-H study was to assess the development of a large group of 
children across adolescence (approximately 10 years; Lerner et al., 2005). These

questionnaires were selected based on an extensive literature review conducted to find measures that would be appropriate indicators of PYD (Lerner et al., 2005). An initial pilot study was done to assess the selected measure, and this resulted in an initial measurement model that was then tested in the first wave of $4 \mathrm{H}$ data (see Lerner et al., 2005). The authors then categorized each of the measures into one of the PYD constructs. The factor structure has been validated and has shown to hold across the adolescent time period (Bowers et al., 2010; Jelicic et al., 2007). A short and very short measure of the 5 Cs has also been developed for use with older adolescents. However, the authors caution against the use beyond factor analysis or structural equation modeling and recommend using a bi-factor model of PYD in such analyses (Geldhof et al., 2014).

Heck \& Subramaniam (2009), conducted a review of PYD programs and recommended the use of the $5 \mathrm{C}$ model due to its empirical support and the fact that the factor structure has been validated. Indeed, the $5 \mathrm{C}$ approach has received the most attention from a research perspective and most of this attention has come from Lerner and colleagues and the longitudinal study funded by the $4 \mathrm{H}$ club of America (Lerner et al., 2005). The fact that a model has received the most empirical support is a valid argument to recommend its use. However, the nature of the research conducted with the model should be examined, as mere use does not necessarily equate to suitability. Again, the $5 \mathrm{C}$ model of PYD is outcome focused. It is argued that programs should be designed and structured in such a manner to develop each of the 5 Cs (Roth \& Brooks-Gunn, 2003) but there is no consideration of how the development of these factors occurs. Thus, there is no discussion of the process, or what occurs within a specific activity context. 
Research using this model does suggest that more activity involvement is related to greater PYD. Agans et al (2014) report that adolescents who engaged in multiple activities (ranging from youth development programs to music to religious groups), reported higher PYD scores (Agans et al., 2014). And likewise, dropping out of some of these activities was associated with more negative behaviors, such as higher depressive symptoms, lower PYD scores, and lower contribution scores (Agans et al., 2014). This suggests that providing youth with a variety of developmental opportunities (i.e., extracurricular activities) is important for the development of positive outcomes, such as those associated with the 5 Cs. Specific to sport involvement, a study by Agans and Geldhof (2012), showed that both male and female adolescents who participated in team and individual sports reported higher PYD (i.e., $5 \mathrm{Cs}$ ) and contribution scores as compared to those who did not participate in sports. For males, there was also a significant relation between team and individual sport participation and decreased depressive symptoms, demonstrating once again that varying activities and contexts promotes the development of positive outcomes.

Several aspects of youth experiences/characteristics have been shown to predict the 5 Cs. These include hope (Schmid et al., 2011), identity styles (Crocetti et al., 2014), flow (Tavares \& Freire, 2016), weight-related behaviours (Fay \& Lerner, 2013) and selfregulation (Zimmerman et al., 2008). In each of these cases, the more positive the experience/characteristic, the higher reported PYD scores.

It is important to point out that the $5 \mathrm{C}$ conceptualization of PYD is an outcomebased approach. There is no consideration given to the process by which these outcomes are achieved or promoted. This leaves out a large piece of the puzzle and one that the 
present study aimed to address. What does PYD in a particular setting, such as sport, look like? According to Lerner, the outcomes can be identified as the 5 Cs but what about the other factors at play? Understanding what factors are associated with PYD outcomes or what experiences in sport are important for PYD outcomes will provide a more holistic understanding of PYD in a sport context.

Initiative, Motivation, and the Youth Experience Scale. Larson's conceptualization does take on more of a process-based approach to understanding PYD. This conceptualization stems from the notion that adolescents are important players in their own development. Essentially, humans are driven to find opportunities to enhance their skills and abilities in various domains and, as such, are driving forces in their own development (Larson, 2006). For positive development to take place, there must be an element of motivation and that motivation must be intrinsically based (Larson, 2006). Through this intrinsic motivation, adolescents gain a sense of agency (i.e., initiating action) and can seek out environments that provide positive developmental opportunities (Larson, 2006). In contrast to the 5C approach, Larson's approach is looking more at the contextual mechanisms that promote positive developmental outcomes.

According to Larson, one of the obstacles associated with development, is getting the adolescent to become intrinsically motivated to produce agency. There are various factors that come into play in that regard. Adolescents need to be able to determine what they want to do and how they will do it. This requires an understanding of the goal they want to achieve, the mechanisms at their disposal to work toward that goal, and an understanding of how they will navigate the pathway to their goal (Larson, 2011). If a goal is deemed too challenging, this can cause the adolescent to experience negative 
emotions, such as anxiety (Larson, 2011). Conversely, if a goal is deemed too easy, this can lead to boredom (Larson, 2011). Thus, the challenge needs to be sufficient to maintain interest but not so challenging as to overwhelm the adolescent. This speaks to the importance of "fit" between an individual and context. From a sport context, when teaching a skill, you want to match the skill to the ability of the athlete. You would not teach a beginner an advanced skill, nor would you teach an advanced player a beginner skill. It might also be difficult for a highly skilled player to stay motivated in a house league setting. There needs to be an offering of the right amount of challenge to keep the player motivated and not bored. It has been noted that motivation is something that can be developed if not present at the beginning of a project (Dawes \& Larson, 2011). This may require the adolescent to find purpose to what they are doing and bring their own sense of connection to what they are trying to accomplish (Dawes \& Larson, 2011).

Youth programs can offer a context for a specific type of PYD, such as emotional development (e.g., experiencing anxiety in a stressful situation and/or happiness associated with doing well; Larson, 2011). It is important to note that Larson refers mostly to non-sport activities when referencing youth programming in this regard (e.g., church groups, theatre, academic clubs). The benefit of such programs, in contrast to sport, is the opportunity for adolescents to have more control over the projects they undertake (Larson, 2006, 2011). For example, with a church group there would be an initial desire to join the specific group knowing who the other members are and what the objective of the group is. Furthermore, as a member of a church group there would be more input on what approaches to take and what roles each member would be assigned. In contrast, with sport, there are try-outs or sort outs for most sports and individuals are 
assigned to a team. This team has a coach who determines the roles that team members will play and dictates how the team will play. However, there are overlapping characteristics of these settings that make it relatable to a sport setting. For example, in both structured sport and non-sport contexts there are adults who can model behaviour and provide safe environments in which to learn.

Larsen (2011) suggests that understanding emotions is an important part of adolescent development - being able to identify one's own emotions and knowing how to react in emotional situations. Sport offers a context where adolescents can experience both emotional highs and lows and must learn to appropriately deal with these emotions. It is also an ideal context for adults to model the appropriate emotional response and behaviour in a given situation. For example, if a child is cut from a team, the parent can express disappointment and help the child decide how they will improve for next season. This shows the child that it is okay to be disappointed after being cut from a team and that they can act to improve their chances of making the team the next year. That said, there are many who would argue that sport is the last place adolescents should look for appropriate emotional responses from adults, as coaches and parents can often be seen yelling at referees in response to a call on the field or ice. Perhaps that is one area where PYD research can assist with informing parents on the importance of modeling appropriate behaviour.

Initiative is a vital part of youth development (Larson, 2000). Basically, adolescents need to develop initiative if they want to be able to develop and execute a life plan (Larson, 2000). Larson defines initiative as "the devotion of cumulative effort over time to achieve a goal" (Larson, 2000, p. 172). Learning to do this will help an adolescent 
navigate real world problems and be successful in adulthood (Larson, 2000). An adolescent needs to be able to commit to a goal, determine the necessary steps to achieve this goal, and put a plan into action that will allow them to reach their goal. This would, naturally include being motivated to achieve said goal and being engaged in the process of achieving it. As there are challenges in life, working towards goals will likely include adjustments and setbacks that require the adolescent to overcome to continue towards their goal.

This idea of the development of initiative fits well with the design of sport contexts. There are multiple goals that can be set within sport, such as skill development or performance goals. To take a performance goal as an example, an adolescent golfer may decide she wants to shoot a score of 75 . She would have to evaluate her current skill level and determine what elements need work and then devise a plan to improve those skills. This could include extra practice times or taking a lesson. Feedback would occur after playing a round and give her an idea of the progress she has made. If it becomes clear that improvement is not being made and the goal is in jeopardy, she may adjust her practice schedule or perhaps alter the goal to be more reasonable. In a team setting, the development of initiative may be more dependent on whether the individual athlete has developed goal-setting strategies, as coaches tend to be the ones who set team goals. The notion of goal setting will be discussed in more detail later in this document.

As a final note, regarding Larson's approach, Larson makes reference to the "real world" and importance of preparing youth for real world experience. He suggests that the value of youth experiences in extracurricular activities lies within the abilities of the program to properly prepare youth for adulthood and the "real-world". This would 
include giving youth the opportunity to problem solve, overcome obstacles, and work toward goals. This is an important argument regarding the present study and the belief that experiences in sport can provide such opportunities. Thus, Larson's perspective may not perfectly encapsulate PYD in sport, but it does provide important characteristics to consider when examining PYD in the sport environment (i.e., initiative, emotional regulation, motivation) and speaks to the value of experiences in youth sport.

To assess developmental experiences in different activities, Hansen and Larson (2003; 2005) developed the Youth Experience Survey (YES). The YES, and current incarnation YES 2.0, measures high-school-aged students' developmental experiences in organized activities (Hansen \& Larson, 2005). As such, the YES would, seemingly, represent process-related constructs as opposed to outcomes.

The original YES consists of 89 items that corresponds to domains of personal development and interpersonal development. Within these domains, there are 17 subscales that load onto six higher order factors of identity work, initiative, basic skills, teamwork and social skills, positive relationships, and adult networks and social capital. There is also a negative experiences factor that includes subscales of social exclusion, negative influences, stress, negative group dynamics and inappropriate adult behaviour. The YES asks participants to think about their current or recent experience in the specific activity of interest and then answer the questions as they relate to the activity. For example, "I became better at giving feedback" or "Learned I had a lot in common with people from different backgrounds". Participants respond on a 4-point likert scale ranging from yes, definitely to not at all.

The YES 2.0 was then created to shorten the original scale and make it more 
psychometrically sound (Hansen \& Larson, 2005). The authors did subject the YES 2.0 to statistical analyses including assessing means, standard deviations, and intercorrelations before conducting confirmatory factor analyses and arriving at the present factor structure. The YES 2.0 has 70 items loading onto the same higher order factors of identity work, initiative, basic skills, teamwork and social skills, positive relationships, and adult networks and social capital. However, after analysis of the factor structure, two overarching domains of positive and negative experiences have been retained instead of the domains representing personal development and interpersonal development.

In what is, arguably, the most valuable research to have been conducted with the YES, Larson et al., (2006) compared the developmental experiences of 2280 youth involved in different extracurricular activities. The authors found that participants involved in sports reported higher initiative, emotional regulation and teamwork experiences when compared to other organized activities (e.g., dramatic arts, religious groups, community groups). This study was only looking at differences between various extracurricular activities on PYD outcomes as defined by the YES 2.0 (i.e., the more positive developmental experienced by youth, the better). This is in line with the expectations of the sport environment discussed previously.

Blomfield \& Barber (2011) have also reported significant developmental experiences in extracurricular activities using the identity exploration and goal setting factors of the YES 2.0. Essentially, an adolescent who has positive experiences in extracurricular activities, as measured by the YES 2.0, will show more positive outcomes, such as, self-worth, academic self-concept, and social outcomes (Blomfield \& Barber, 2011). These studies demonstrate two important things. The first being that 
extracurricular participation is valuable for youth and the second being that sport represents a context with added benefits for positive developmental outcomes (e.g., promotes initiative).

There are relatively few studies that have used the YES 2.0 in its entirety. Most studies reviewed have used subscales as they relate to the purpose of their study. For example, studies looking at academic outcomes have used just the academic initiative subscale items (Danielsen et al., 2010, 2011). Blomfield et al (2012), conducted a study looking at peer relationships, identity exploration, and structured leisure activities using the appropriate subscales from the YES 2.0 to measure peer relationships and identity exploration. Although limited, much of the work using the YES 2.0 has been done outside of a sport setting or from a comparative perspective (i.e., how do activities differ). The way in which the scale has been used may reflect the utility of it, basically suggesting that the scale is not an effective way to assess developmental experiences but rather as a tool to measure specific developmental outcomes.

Looking at the factors of the YES, it could be argued, and has been by Holt et al., (2017), that many of the factors of the YES are, in fact, dependent/outcome variables as opposed to independent or predictor variables. The YES is supposed to offer a way in which to assess experiences in youth activities. However, the mix of questions related to process and outcome expectations calls into question the utility of the YES in this regard. It could be argued that the constructs represent two separate types of variables. Goal setting (a component of initiative in the YES 2.0), as discussed by Larson, is an important part of development and represents a key experience in sport. On the other hand, teamwork and social skills and basic skills represent the intended outcome of 
participation in sport as outlined by PYD theory. Essentially experiences in youth activities are intended to lead to the development or enhancement of these skills. As it is presented, the YES 2.0 measures more outcome variables than process or predictor variables related to participation in an activity. Therefore, although it does assess developmental experiences, the YES does not offer a way in which to understand how specific experiences in sport are associated with positive developmental outcomes.

\section{A Sport-Based Model of PYD}

As is evident by the PYD literature, there is a great deal of variability in how it is discussed, modeled, and assessed. Holt and colleagues (2017) point out that both previously mentioned conceptualizations of PYD were not developed or designed to be used in a sport context, even though they are often used in a sport setting. As such, Holt et al., (2017) recently proposed a sport-based model developed through a summation of 63 qualitative research articles (see Figure 1). The research articles used in the review had to at least mention PYD in some area of the article. Thus, some articles would be more relevant than others as research using theory of change or life skills transfer were included because of their conceptual similarities and references to PYD. This is a reflection on the state of qualitative PYD literature in sport and the varied approaches used by researchers. Nevertheless, the model presents a viable outline for the development of a testable, useful model for the study of PYD in sport. Although there are aspects to the model that may require modification, it does offer a sound starting point and valuable insight into how PYD in sport should be assessed.

\section{PYD Climate}

A key aspect of the model is the distinguishing of the PYD climate and PYD 
outcomes. This, in a way, links the basic ideas of Larson and the $5 \mathrm{C}$ approaches with both the contextual (i.e., process-related) factors and outcomes considered. This is a rather novel and a very valuable contribution to the PYD literature. The developmental experience in a sport environment, such as goal setting, leads to a positive developmental outcome, such as initiative.

\section{Figure 1}

A Sport-Based Model of Positive Youth Development

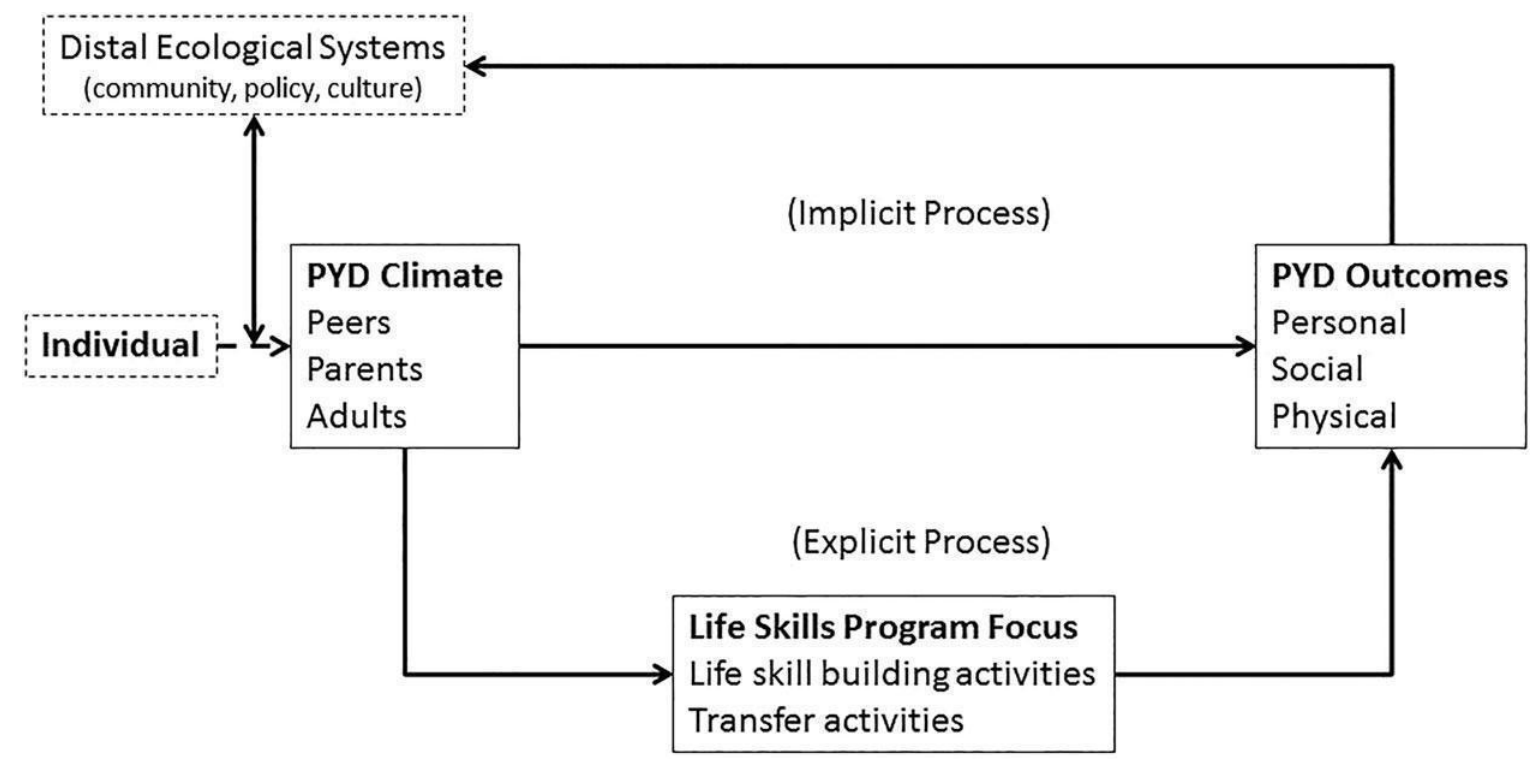

Note. Reprinted from Holt et al., (2017). A grounded theory of positive youth development through sport based on results from a qualitative meta-study. International Review of Sport and Exercise Psychology, 10(1), 1-49.

As the studies included in the review were of a qualitative nature, Holt et al., (2017) looked for consistent themes and found three important categories for both the PYD climate and PYD outcomes. The PYD climate referred to relationships within the sport context that would assist with the creation of a climate to promote the development of PYD and PYD outcomes. The first important relationship identified was that of 
coaches/leaders and having a strong relationship between the athlete and coach. The second relationship identified was between athletes and their peers/teammates. Holt et al., identify the enduring nature of these relationships as being a particularly salient feature in promoting PYD. And the final relationship was that between the athlete and his/her parents. These relationships and the value placed on them for creating a positive sport climate is consistent with developmental theory and the importance of developing relationships with significant others in a PYD context. Furthermore, a great deal of research has demonstrated the importance of coach and teammate relationships to a variety of outcomes in sports. The following sections provides an overview of the literature on coach and teammate relationships in a sport setting.

The Importance of Coaches. An important quality of a PYD program is the involvement of a significant adult relationship (e.g., Benson et al., 1998; Roth \& BrooksGunn, 2003). As mentioned, coaches represent this key feature in sport and, as such, the quality of the relationship between coach and athlete is valuable. In a study by Vella et al., (2013), the best predictors of PYD outcomes were a transformational leadership style and a good relationship between the coach and the athlete. Coaches represent a central figure in a sports environment. Coaches are responsible for many of the rules, regulations, and experiences that an athlete will be exposed to when participating in their specific sport. A coach runs practice, determines starting lineups, dictates the structure of play (e.g., methods of playing defense), and how the team will operate (e.g., dress codes). Coaches are continually communicating with players, such as, addressing errors in play, teaching a new skill or developing a skill, communicating how a drill should be carried out, or encouraging a team before a big game. These represent only a few of the many 
ways that a coach can interact with a player.

PYD programs have identified the importance of structured, adult-led activities. The coach-player relationship represents a key adult relationship, distinct from a parentchild relationship. Within the limited scope of PYD and sport, coaching has received some attention from researchers. Of value is the finding that both coaches and athletes believe that coaching can influence developmental outcomes (Camiré et al., 2012). This is an important finding as it confirms a few important assumptions. First, athletes view coaches as influential players in the sport context. Secondly, both recognize the ability to produce change through actions and interactions. Coaches feel they can foster growth and development. Athletes believe that coaches can assist in the development of important characteristics and also recognize that they, themselves, can change. This demonstrates an implicit buy-in for coaches and athletes and this is an essential point to be made. If change/development is to occur, it must be believed that it can occur and that actions being taken are purposeful and effective.

Specific behaviours have been shown to be more effective in fostering positive outcomes. From a more general perspective, authoritative behaviours appear to be the most effective. Coaching that emphasizes support, instructional behaviours, and communication is associated with more positive developmental experiences (Smoll et al., 1993; Vella et al., 2013; Watson et al., 2011). Smoll et al., (1993) conducted a study comparing a group of coaches who received training on effective coaching strategies (e.g., supportive, authoritative) to coaches who did not receive training and found that athletes who played for the trained coaches reported more favourable experiences (e.g., had more fun). This is interesting but not the most valuable piece of the study. The 
authors also reported that boys who reported low self-esteem at the beginning of the study showed an increase in self-esteem scores if they played for trained coaches (Smoll et al., 1993). Similar findings were reported by Smith et al., (2007) who found that sport anxiety significantly decreased across a season for players of trained coaches. These findings highlight not just the value of positive coaching behaviours but also the fact that coaches can enhance player experience and promote positive psychological outcomes for youth.

Behaviours also associated with positive outcomes are autonomy supporting behaviours (e.g., seeking input from the athlete, showing interest in emotional well-being of the athlete; Cronin \& Allen, 2015) found a positive association between autonomy behaviours and the 5 subscales of the YES-S. Coatsworth and Conroy (2009) tested a similar association using needs satisfaction and found a positive association between autonomy behaviour and basic psychological needs. More autonomous behaviours predicted greater satisfaction of basic needs and this, in turn, predicted positive developmental outcomes, such as competence and initiative (Coatsworth \& Conroy, 2009). Larson identified the development of initiative as a key developmental task for youth. Behaviours that support autonomy would, likely, similarly support the development of initiative. In fact, Cronin and Allen (2015) list allowing an athlete to take initiative as an example of autonomy supporting behaviours.

Research has consistently shown an association between the type of coach behaviour and psychological outcomes for youth athletes. One interesting association is that of positive feedback/praise and positive outcomes. Coatsworth and Conroy (2009), found that students in a summer swimming league who received praise, reported higher 
levels of competence and relatedness. More positive feedback has also been linked with higher intrinsic motivation (Koka \& Hein, 2003), perceived competence (Allen \& Howe, 1998), and continued participation (Mouratidis et al., 2010) in sport-based settings. This extends to global psychological outcomes, such as well-being or ill-being (Mouratidis et al., 2010). Interestingly, it would appear that the influence of the type of feedback can also be dependent on the difficulty of skills being learned. In a study by Tzetzis et al., (2008), positive feedback was more strongly associated with self-confidence when the skill being taught was considered easy. For more difficult skills, corrective feedback was desired from the athletes.

In a study of college-level athletes, Amorose \& Horn (2001) found coaching behaviors to predict intrinsic motivation. Specifically, coaches who demonstrated more instructional and training behaviour had athletes with higher reported intrinsic motivation. From an opposing perspective, negative behaviours have been similarly associated with negative outcomes. Baker et al., (2000) found that negative personal rapport was related to athletes' anxiety levels. The authors postulate that coaching behaviour can be a contributing source to athletes' experiences of sport anxiety. Taken together, this research demonstrates the significant influence that coaches have on athletes and athlete experiences in sport.

As mentioned previously, coaches themselves view their behaviours as influential. Gould et al., (2007), interviewed football coaches who had a lengthy career and were considered successful. The coaches highlighted the importance of relationships with the athletes but also spoke to the value of other behaviours. One particularly salient experience identified by the coaches was the setting of both short and long term goals and 
helping the athletes in pursuit of these goals. This harkens back to Larson and the value placed on setting goals and devising strategies to achieve these goals as instrumental to development. The coaches further commented on how, within the context of the football field, they were able to reinforce specific values such as discipline and work ethic (Gould et al., 2007). This just serves to punctuate the influential nature of coaching relationships and potential they have for enhancing youth experience in sport and promoting positive developmental outcomes for youth.

It is evident, based on the literature, that coaches represent important relationships for youth involved in sports. The PYD literature specifically identifies the value of relationships with significant adults in the promotion of positive outcomes. As such, consistent with the sport-based model of PYD, in the current thesis, it was anticipated that these relationships would play a role in how experiences in sport predict developmental outcomes. The more supportive or positive the coaching behaviour, the more likely a youth is to experience positive outcomes.

The Importance of Teammates/Peers. Perhaps surprisingly, peers have not received a great deal of attention as social agents in sport (Smith, 2003). This is an oversight in the literature as peers are, undoubtedly, an important part of the sport context and represent key relationships to athletes. Peers are included as an important part the PYD climate in the sport-based model of PYD and should be considered as influential factors in the experiences that youth have in the sport context.

A teammate relationship is a rather unique relationship. Regardless of level of play, teams spend a good deal of time together across a season. This is often in pursuit of a common goal (i.e., to win) and requires a coordination of effort. With a team sport, 
each member must do their part to contribute to this common goal. This requires, on some level, getting along with teammates and supporting them regardless of what is happening off of the playing surface and speaks to the value of developing strong interpersonal skills. Sport provides athletes with the opportunity to develop and maintain friendships as well as to learn how to get along with others. This can extend beyond teammates to opponents, as well. Learning to respectfully communicate and resolve differences in a prosocial manner is a valuable developmental tool.

Teammate relationships are associated with a number of outcomes in sport. Friendships and positive relationships with peers in sports predict continued involvement in sport, more enjoyment, a greater level of effort and more value associated with participation (Ullrich-French et al., 2011; Ullrich-French \& Smith, 2006). Friendships can be made and maintained within a sport setting (Weiss et al., 1996) and children in sport are often accepted or rejected based on athletic abilities/competence (Bigelow et al., 1989; Weiss \& Duncan, 1992). In fact, Smith et al., (2006) found that lower group acceptance in sport translated to higher anxiety, lower perceived competence and lower enjoyment. To further demonstrate the importance of peers in sport, peer acceptance was found to fully mediate the association between participation and global self-esteem for adolescent athletes (Daniels \& Leaper, 2006). This is in line with findings showing team acceptance to predict self-esteem and depressive symptoms for adolescent participants of team sports (Hill, 2013).

Teammate behaviour in sport can also have an influence on the experiences of youth. Teammates who are prosocial are positively associated with enjoyment and effort (Al-Yaaribi et al., 2016). Conversely, a teammate displaying antisocial behaviours is 
associated with decreased effort and commitment to the team (Al-Yaaribi et al., 2016). Supportive teammates have also been shown to be related to greater commitment for high level swimmers (Santi et al., 2014) and assisted with overcoming adversity in NCAA division 1 athletes (Morgan \& Giacobbi, 2006). Although, limited, research does suggest that teammates play a role in the experiences of athletes in sports.

One way that this has been investigated in the sport psychology literature is through motivational climate research. This approach stems from achievement orientation theory proposed by Nicholls (1989) whereby athletes can be classified as either ego or task oriented. A task-oriented person is primarily focused on skill development and views success as the improvement in his/her skill. Conversely, an ego-oriented person is more focused on achievement and views success as performing better than others. A taskoriented perspective is considered to be more adaptive and is associated with more positive outcomes for athletes, such as setting more challenging goals and increased effort (Le Bars et al., 2009; Ntoumanis et al., 1999).

In terms of assessing teammates and the role that these individuals can play in enhancing or hindering developmental outcomes, looking at peer motivational climate offers an intriguing way to investigate this relationship. Peer motivational climates refer to the team environment as a whole and an individual athlete's perception of how teammates/peers view their achievement goals. Researchers have found that teams who are perceived to be more task-oriented are more likely to be associated with positive outcomes for the individual athlete. These include greater enjoyment and effort (Vazou et al., 2006), higher intrinsic motivation (Hein \& Hagger, 2007), and commitment (Ntoumanis et al., 2012). Conversely, a team with more of an ego-oriented climate can 
produce antisocial attitudes (e.g., cheating; Ntoumanis et al., 2012) and lead to negative relationships with teammates (e.g., conflict with teammates; (Ommundsen et al., 2005). Again, there is a limited amount of research assessing the nature of teammate relationships in sports and how those may be associated with developmental outcomes. However, teammates represent an important social relationship for young athletes. This may be particularly true for adolescent athletes who are at a time in their development when peer relationships become increasingly important (LaFontana \& Cillessen, 2010; Larson \& Richards, 1991). And it does appear that these relationships can serve to help or hinder the athlete's experience in sport. As described above, more supportive behaviours and attitudes are associated with more positive outcomes.

It is important to address the fact that parents have been excluded from this discussion. Holt et al., (2017) identified parents as a key relationship within the PYD climate and it is acknowledged that parents represent an important and influential relationship. Primarily, parents are viewed as important sources of support for children in sport. This includes in terms of success (Gould et al., 2006), moral development (Stuart \& Ebbeck, 1995), enjoyment, self-determined motivation, and competency beliefs (Ullrich-French \& Smith, 2006). Parents have been excluded from the present study for several reasons. As discussed previously in this document, PYD theory emphasizes the value of a relationship between a youth and an adult community member, such as a coach. This is a separate relationship from that of parent-child, and, when looking from a PYD perspective, the nature of this youth-adult relationship is of primary interest. Another reason for exclusion relates to the specific focus on experiential factors within the sport context. When participating in a sport, the more immediate relationships 
are with coaches and teammates. As such, these relationships are more likely to influence the experiences an adolescent has in sports. Although there may be a number of relationships that could influence youth outcomes (e.g., friends, siblings), the present study focused on those factors that are directly involved in the participation of the sport (e.g., on the field of play).

\section{PYD Outcomes}

Holt et al., briefly summarized the PYD outcomes into three domains: personal, social, and physical. Each of these domains is consistent with the PYD literature and outcomes described by previously discussed conceptualizations of PYD. In addition, many of the attributes discussed within the domains are consistent with research regarding positive outcomes associated with physical activity and sport involvement. The outcomes are quite general and demonstrates the variability of the PYD literature and the many outcomes that can fall within PYD. That said, each domain can be broadly associated with the attributes outlined in the models of PYD (e.g., the 5 Cs).

Within the personal domain, positive self-perceptions were the most commonly reported outcome. This encompassed a wide variety of personal characteristics, including academic achievement, self-esteem, independence, respect, and learning to work hard. The outcomes associated with the personal domain are broadly consistent with those posited by PYD theory. Achievement, self-esteem, and learning to work hard can be compared to Lerner's concepts of competence, confidence, and character. Furthermore, independence is a key facet of how Larson conceptualizes PYD through autonomy.

The social domain included attributes associated with meeting new people and making friends as well as the development of communication skills. Social relationships 
have been well-documented as a benefit to participating in sport and one of the reasons that many engage in organized sports (Eime et al., 2013). Social domain outcomes are also consistent with the connection facet described by Lerner where developing connections to significant others is an important indicator of PYD. Key social connections described by Holt et al., include teammates but also extend to coaches and other important adults in the community. As previously mentioned, the sport environment provides a perfect context in which youth can develop social relationships and learn the skills to communicate in a pro-social manner (e.g., encouraging teammates).

Not unexpected, an important outcome of sport participation is physical competence and the development of physical skill. The ability to develop skill in a given sport and to feel competent in that skill is a key developmental outcome. Stemming from Bronfenbrenner, the development of competence has repeatedly been discussed as an important part of PYD. As previously mentioned, sport provides an ideal setting in which to learn new skills, develop competence, and to demonstrate that competence through games/competitions.

Not much detail is presented within the PYD outcomes portion of the model. However, it provides a testable guide as to what should be assessed when looking at PYD outcomes in sport. Furthermore, the key outcomes described by the Holt et al., model are consistent with PYD literature and the outcomes commonly assessed in a PYD framework. Thus, Holt et al., have provided a useful model to assess PYD in terms of the climate and outcomes. However, there appears to be an important aspect to be considered in the sport-based model of PYD and in the sport-based PYD literature - there are specific experiences provided in a sport context that may help to promote PYD outcomes. 


\section{Experiences in Sport as the Missing Factor}

As mentioned, the sport-based model of PYD offers a way to assess PYD in sport. The separation of climate and outcomes connects the conceptualizations of PYD that have focused mainly on outcomes associated with PYD. However, there is still an aspect of the model and the conceptualization of PYD in sport that is missing. Relationships and the sport climate are important to create the developmental context in which PYD outcomes can be promoted, but there are a number of other important experiences that need to be taken into consideration. How does goal setting in sport assist development? If an athlete is cut from a team or wins a championship, how do these experiences shape developmental outcomes? These are questions that have yet to be empirically examined. This is a key point to be made as the present research was interested in the experiential factors associated with sport and how these factors influence developmental outcomes. There are a myriad of important experiences that an athlete engages in regularly that could aid in PYD.

Along with the proposed model, Holt and colleagues (2017) offer the following definition of PYD in sport, "PYD through sport is intended to facilitate youth development via experiences and processes that enable participants in adult supervised programs to gain transferable personal and social life skills, along with physical competencies. These skill and competency outcomes will enable participants in youth sport programs to thrive and contribute to their communities both now and in the future" (Holt et al., p. 231). The definition refers to experiences and processes, but the model does not include the specific experiences an athlete will have in a sport setting. Informed by the developmental theory and sport literature, three specific experiential factors 
associated with the sport experience, goal setting, adversity, and skill development, are discussed below and were the primary focus of the youth experiences in sport scale development. The development of such a scale was intended to provide a way in which to assess the association of developmental experiences in sport with positive youth development outcomes.

The Experience of Goal Setting. As was previously discussed, Larson identifies goal setting as a valuable process for youth. Youth need to understand the types of goals they want to achieve, how they can go about achieving these goals, and what level of challenge should be associated with these goals (Larson, 2011). Larson speaks of the value of goal setting for enhancing motivation, but goal setting may also be valuable to the development of other positive outcomes for adolescents.

Danish et al., (2003) state that the major benefit of sport is the opportunity to attain one's personal goals. Goal attainment is a valuable experience in adolescent psychosocial development (Danish et al., 2003) and has also been linked with identity development (Marcia, 1966) and self-regulation (Bandura, 1997; Schunk, 2001). It has also been suggested that goal setting can help with adolescent brain development (Scarborough et al., 2010). With regard to identity development, goal setting is a part of the development of purpose and competence which are considered ego strengths under Erikson's model of identity development (Markstrom et al., 2005). Erikson has noted the importance of social institutions to identity development, essentially stating that the development of identity cannot take place without the interactions present in social institutions. Goal setting represents a valuable part of the institution of sport.

There has been research presented in recent years to demonstrate an association 
between goal setting and well-being. Hill et al., (2011), reported a positive association between increases in prosocial goals and well-being for emerging adults. According to Danish and colleagues (2003), sports allow adolescents to gain experience with setting goals and the success that comes along with achieving them. This is beneficial as it allows for youth to see the value of setting goals which may help them in setting and achieving goals in the future. As Hill et al., (2011) reported, this could be quite beneficial for long term development.

As has been mentioned, setting goals helps direct behaviour. Adolescents must determine the goals they want to set, devise a plan to achieve these goals, reevaluate when/if necessary and be prepared to set new goals when previous ones have been achieved. It is easy to see how such a process could lead to positive outcomes. The steps associated with goal setting are similar to those required for the development of selfregulation (e.g., forethought, control, reflection; Scarborough et al., 2010). In addition, achieving goals provides a confirmation that the youth is competent and able to meet expectations. Competence has been repeatedly identified as an important aspect of youth development and, this would likely also lead to enhanced self-confidence. Finally, setting goals helps to develop initiative as youth are required to work towards their goals and find ways to be successful. This could mean going to the driving range to hit a bucket of balls every night or spending an hour shooting free throws.

In order to realize the benefits associated with goal setting, there are certain features that should be present in the goals and goal setting strategy. Primarily, goals should be specific, difficult, and represent a varied focus (Burton \& Weiss, 2008; Kingston \& Wilson, 2008). In addition, both long term and short-term goals should be 
incorporated with the ideal strategy using short term goals to achieve long term goals (Locke \& Latham, 2006). As pointed out by Danish et al., (2003), goals in sport represent these features with most goals in sport being short term, measurable, and tangible. Furthermore, a mixture of process, performance, and outcome goals should also be incorporated (Filby et al., 1999). An example of these for a young golfer would be to set a goal to bring the golf club straight back on the takeaway (process), and a goal to hit 14 of 18 greens during a round (performance) and then to shoot 78 (outcome). When developing goals, it is important for the athlete to have considerable input into their goals (Gallucci, 2014). This gives the athlete a sense of ownership over their goals and encourages independence. As a final note, seeking input and feedback from others can also be helpful to establishing appropriate goals (Gallucci, 2014). A parent or coach can offer more objective advice as to whether the goal is attainable, easily measured, or perhaps not challenging enough.

Intuitively, research has shown that the types of goals and motivations behind the types of goals set can influence outcomes. Goals are not always comparable or equally meaningful and can be described as existing in a hierarchy with more important goals at the top (Wrosch et al., 2003). According to Wrosch et al., (2003), the highest goals are those that are most important to one's sense of self. Depending on the type of goal, internalizing (becoming central to the self) is an important aspect of goal striving as this helps motivate and direct attention (Ntoumanis et al., 2014). As mentioned, goals should be autonomous and set by the adolescent whenever possible. However, goals can also be internalized if set by others (Ntoumanis et al., 2014). These are important points to consider as the purpose of goals and the motivations behind specific goals play a role in 
the effectiveness of this behaviour and realization of benefits associated with goal setting. Generally, more autonomous goals have more favourable outcomes in terms of persistence and affect (e.g., Ntoumanis et al., 2014). In addition, the ability to achieve certain types of goals can have implications for outcomes including what happens if an individual fails at achieving a goal. An adolescent needs to be able to persist when goals are challenging and also disengage from goals when it is evident that the goal is not achievable (Ntoumanis et al., 2014).

Research in goal setting and sport is frequently conducted within the framework of maximizing athlete performance and not necessarily with developmental outcomes in mind. Goal setting research in sport has consistently demonstrated the value to skill development and self-efficacy (Schunk, 1983), improved performance (Filby et al., 1999; Mellalieu et al., 2013), greater effort, and commitment (see Kingston \& Wilson, 2008, for a review). A study of 328 Olympic athletes found that all used goal setting and that they reported this to be a highly effective way to enhance performance (Weinberg et al., 2000). For these athletes, setting goals that challenged them to go above their level of performance ability were preferred (Weinberg et al., 2000). Studies have also shown that athletes who have not previously set goals will see an improvement in performance when they set specific goals (O’Brien et al., 2009; Swain \& Jones, 1995).

Goal setting has been consistently linked with success in a variety of achievement-based settings, including sports, academics, and the workplace (Burton \& Weiss, 2008; Gallucci, 2014). Goal setting represents a valuable tool for athletes. There are a number of stipulations associated with this notion, but, ultimately, goals aid with a variety of positive outcomes (e.g., increased effort, better performance) for those who 
effectively engage in the practice (Burton \& Weiss, 2008; Gallucci, 2014; Locke \& Latham, 2006). Looking beyond the sport environment, a study of students playing a computer game found a significant, positive association between learning goals (as opposed to no goals or performance goals) and the amount of fun reported by the participants (Nebel et al., 2017). Enjoyment in sport has been linked to continued participation for adolescent athletes (Scanlan et al., 1993) and this research shows that goals can be used to enhance the developmental experience and promote positive outcomes.

A valuable benefit of goal setting is increased motivation (Burton \& Weiss, 2008). A particularly interesting study by Elliot and Harackiewicz (1994) looked at goal setting, intrinsic motivation and achievement orientations. The authors found that achievement orientation moderated the association between goal setting and motivation. Specifically, mastery focused goals predicted higher levels of intrinsic motivation for those who were low in achievement orientation (Elliot \& Harackiewicz, 1994). Such results are interesting for a few reasons. First, it demonstrates the importance of considering individual characteristics as moderating factors. And, second, it demonstrates Larson's contention that intrinsic motivation can be developed. Finally, this highlights just how important it is to look at experiences within sport to truly understand how sport promotes PYD outcomes.

Goal setting appears to be the impetus for a number of positive outcomes associated with athletic endeavours. As such, it is necessary to examine this construct as an experiential and influential factor in sport and not as an outcome of participation itself. If it can influence development, then this needs to be assessed so that coaches, parents 
and athletes can be trained to effectively promote effective goal setting strategies. Larson identifies the importance of adolescents learning how to set and work towards goals in life. Allowing youth to gain these skills and practice them in sports represents a tremendous opportunity and lends a great deal of support to the idea that goal setting in sport represents a key developmental experience.

Experiences with Adversity. Facing adversity is a difficult task for anyone. It may be even more difficult for youth who do not possess the necessary tools to adequately cope with adversity. However, sport, as a potential microcosm of the real world, may help youth develop these vital skills through the challenging experiences present in sport. These range from somewhat simplistic challenges, such as struggling to master a new skill, to more serious challenges, such as suffering an injury. Adversity in sport can also include experiences such as losing an important game or being cut from a team. Although it may seem counter intuitive to wish adversity on youth, research suggests there are benefits to development for those who must face and overcome adversity in sport. This represents a valuable feature that has been overlooked by the PYD literature as a potentially influential variable and explanatory factor in how sport contexts promote PYD outcomes.

Looking at Olympic athletes, it is evident that adversity can breed success. Two qualitative studies assessed experiences of adversity in champion Olympic athletes. In both of these studies, success was partially attributed to having to face and overcome an adversarial situation (Howells \& Fletcher, 2015; Sarkar \& Fletcher, 2017). The types and nature of these stressors varied from external factors (e.g., dealing with a learning disability) to sport-related factors (e.g., conflict with coach, not being selected for a team, 
failing in sport, serious injury). In all of these situations, the athletes were forced to face the adversity, assess and reevaluate their situation, and then, ultimately, experienced growth.

A qualitative study by Wadey et al., (2013), supports the contention that growth stems from adversity. The study asked coaches to reflect on growth experiences of athletes who had experienced an injury in sport. The coaches identified four broad dimensions of growth: physical, psychological, social, and personal. These are obviously quite broad and represent basically all areas of potential growth. What is most relevant with regard to the aims of the present research are the lower-order themes under psychological and personal growth. These included increased emotional intelligence, greater enjoyment in sport, being more organized, more confident, and more focused (Wadey et al., 2013). There is something about facing an adverse situation that allows for an athlete to grow in specific, developmentally important ways. Additionally, in a study conducted by Galli and Reel (2012), athletes reported increased self-confidence and resiliency after facing an adversity. Savage et al., (2017), have gone so far as to suggest that adversity may be beneficial for elite athlete development.

Specific aspects of the sport experience have been identified as key contributors to dealing with adversity. These include a passion for the specific sport and commitment to it (Galli \& Vealey, 2008). These two concepts could be considered components of intrinsic motivation, demonstrating both the value of intrinsic motivation and the interconnectivity of personal characteristics, experiences in sport, and developmental outcomes. This combines some of the ideas espoused by Bronfenbrenner and DST with the incorporation of the experiential component proposed by the present study. An athlete 
experiences adversity in sport and is better able to deal with it because of their commitment to the sport. In almost all cases, athletes felt they were better (i.e., higher perceived potential) as a result of experiencing the trauma. According to the Galli and Vealey (2008), “trauma can offer a change to add, hone, or reinforce one's abilities and apply perspective to development" (p. 114). Again, suggesting that adversity within the confines of sport, can be a beneficial experience for athlete development.

One thing that has repeatedly come up in studies of the effects of adverse situations in sports is the importance of social support. Consistently, parents, coaches and teammates are identified as critical components of overcoming an adverse situation through their support and encouragement (e.g., Galli \& Vealey, 2008; Howells \& Fletcher, 2015; Sarkar et al., 2015). Social support and relationships within the sport environment are consistently identified as valuable relationships for youth. This just represents another area in which parents, peers, and coaches can shape youth experiences and developmental outcomes.

Facing adversity may not be a pleasant thing for an adolescent to endure. However, long term, it appears to be a benefit to development. Within the confines of sport, an adversarial situation can be safely confronted and overcome without serious psychological injury. Although being cut from a team can be devastating, learning to deal with such a situation during adolescence can prepare an individual for adulthood when such situations have more serious consequences (e.g., being let go from a job).

Experiences with Skill Development. Beginning with Bronfenbrenner, the idea of developing/displaying competence in a given area has been consistently identified as a vital part of PYD. It is even included as one of Lerner's 5Cs of PYD. The essential idea is 
that through interactions with the given environment, youth learn, develop and practice skills and, thus, will develop competency in that skill. As skill development is implicit in sport participation, the sport context is ideally structured to allow youth to develop competency in a number of different areas. The most obvious is the development of physical skill. If competence is the desired outcome, then skill development is the necessary antecedent and should be considered as an important causal mechanism within the sport environment.

Empirical research looking at the skill development in youth is sparse. Most work looks at perceived competence as opposed to the process by which competence is developed. If an athlete believes that they are good at playing hockey (competent), how did they get to this point? Is it through the development of skills related to hockey? Intuitively, this makes sense. To become competent at something you must first learn how to do that something. To have a good slapshot, you must first learn how to do a slapshot. Furthermore, how do things like coaching style influence the association between skill development and competence? There is some work to suggest that more positive relationships in sport leads to higher perceived competence (e.g., Ullrich-French \& Smith, 2006). Furthermore, how does motivation affect skill development and competence? An achievement motivation that promotes skill mastery as opposed to performance outcomes will likely have an effect. For example, Tenenbaum \& Eklund (2007), discuss how a task-involved athlete (focus on skill development) uses an undifferentiated approach to perceived ability. This means that the athlete is less concerned about how he/she fares in terms of ability in comparison to her peers and this focus is associated with setting more challenging goals, persistence and higher 
engagement (Tenenbaum \& Eklund, 2007).

The benefits of sport participation often include the opportunity to develop new skills (e.g., Chard et al., 2015). Recently, emphasis has been placed on the value of young children learning fundamental movements. Fundamental movements are basic physical skills, such as throwing a ball or jumping. These basic skills provide the foundation for future physical skill development (Lubans et al., 2010). It is important for young children (i.e., ages 6 to 10) to learn these skills in order to be successful in future physical activity pursuits as failure to develop competence in these fundamental movements makes developing expertise in specific physical skills much more difficult (Balyi, 2001). For example, failing to learn how to properly throw a ball in early years makes it more challenging for a child to learn how to accurately throw a baseball to first base when he/she joins a baseball team.

A framework for athlete development has been implemented in order to maximize skill development and encourage long term involvement in physical activity. Called Long Term Development in Sport and Physical Activity (LTAD; Sport for Life, 2019) the idea is based on the developmental readiness of children and adolescents for aspects of skill development. According to Balyi (2001), athletes under the age of 10 should focus on fundamentals and becoming competent at basic skills. From the ages of 10 to 14 (or 10 to 13 for females) is considered a critical period for skill development. This is when athletes learn how to train and begin to learn the specific skills associated with given sport. Skipping this stage can result in difficulties in skill development and may even prevent the athlete from developing to their full potential (Balyi, 2001). After this stage is complete, athletes from ages 14 to 18 enter the training to compete stage where there is 
equal focus placed on training and competition and all training is specific to the sport.

From ages 18 and up, athletes are in the training to win phase where focus is on competitions and ensuring that peak performances are occurring during competitions (Balyi, 2001). This is considered a late-specialization framework where focus on serious competition comes later and more so in late adolescence and adulthood. This is the pathway for most sports and, particularly, team sports (Balyi, 2001) and attempts to follow developmental trajectories to enhance both experience in sport and the learning of sport-specific skills. Such an idea is also supposed to promote long term involvement in sport and reduce the likelihood that individuals will drop out of sport at any stage.

Other similar frameworks have been proposed, such as the FTEM (foundation, training, elite, mastery; (Gulbin et al., 2013) but they follow similar principles with foundational skill development a priority for younger athletes followed by sport-specific training as the athlete grows. An athlete who is exposed to sport-specific training at a young age, is more likely to experience burnout (Leite \& Sampaio, 2012). This is a common argument in youth sports where highly competitive teams are available for younger and younger age groups. By the LTAD, or similar framework, having an 8-yearold play AAA hockey is not conducive to healthy or successful athlete development. However, this seems to go against the desires of parents and sport organizations. A search of hockey associations in a major urban area found that 3 of the 4 associations all offered novice level (age 7 and 8) competitive teams.

\section{Summary}

Research indicates that experiences with goal setting, adversity, and skill development are salient features of organized sports. The sport-based model proposed by 
Holt et al., (2017) has not yet been subjected to empirical validation and does not offer a way in which to test experiences in sport. What it does offer is the notion that, within the sport setting, there are experiences that lead to outcomes and that social relationships are a key part of the PYD climate. This is a sound starting point and by modifying the model, a more holistic representation of PYD in sport can be developed. To that point, the present study has proposed testing an amended version of the model that includes experiences in sport as an important part of the developmental pathway. This revised model can be seen in Figure 2. As mentioned, the present research is focused in the implicit pathway so the proposed model has omitted the life-skill focused (explicit) pathway and focuses solely on the implicit pathway from the PYD climate to PYD outcomes.

\section{Research Objectives and Study Designs}

The overarching research objective was to better understand the mechanisms through which the sport environment (i.e., PYD climate) promotes positive developmental outcomes. Presently, there are no tools to assess the experiences in sport that are associated with PYD. Using the developmental theories described by Bronfenbrenner, DST, and PYD conceptualizations, along with the sport-based model of PYD, it is possible to get a general sense of what a theoretically sound and empirically valid measure of experiences in sport should include. To appropriately assess the modified sport-based model of PYD, a key aim of the present study was to develop a new measure of youth experience in sport.

To achieve this objective, three studies were carried out. Study One involved the development of preliminary scale items, conducting interviews to better understand the 
types of experiences individuals have while engaging in organized sports, adjustment of scale items and expert review of the ESSY. The aim of Study Two was to assess the factor structure of the ESSY. Study Three had two primary goals. The first was to confirm the factor structure of the ESSY proposed by Study Two. The second goal was to use the ESSY to test the modified sport-based model of PYD presented in Figure 2.

\section{Figure 2}

Proposed Sport-Based Model of PYD

PYD OUTCOMES

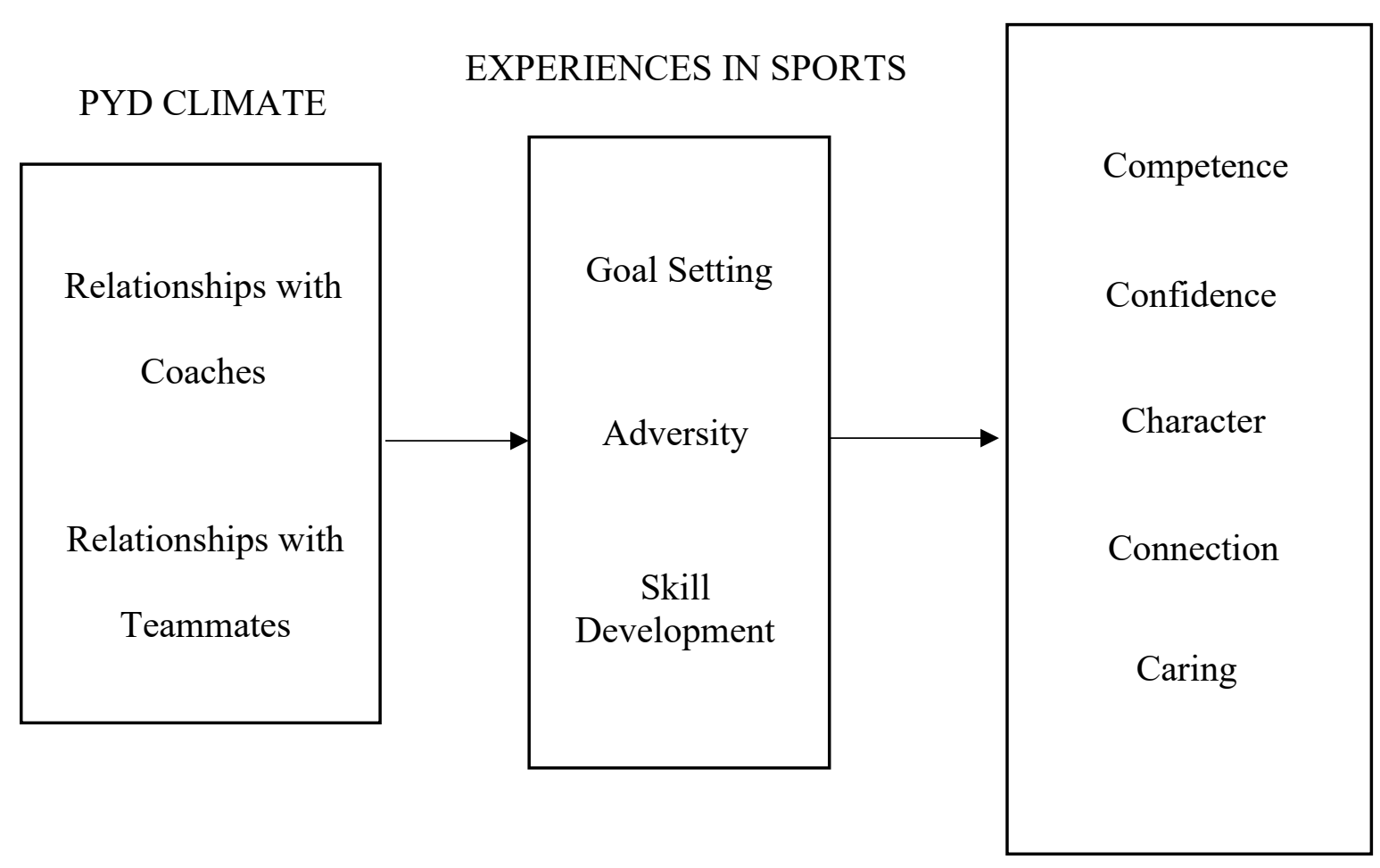

\section{Study One: Scale Development}

\section{Overview}

To develop scale items, Study One involved three important steps beginning with the development of preliminary scale items based on the literature reviewed. To ensure that these constructs were representative of developmental experiences in sport and that 
important aspects of the sport experience were considered, the second step in the process was conducting interviews. Based on the research reviewed, it was expected that interviews would yield information regarding specific experiences in sport from adolescents who participant in organized sports. It was expected that participants would report experiences consistent with goal setting, facing adversity, and skill development. However, it was also expected that additional features of organized sports would be reported providing greater understanding of the types of experiences that are prevalent and meaningful to adolescents engaged in organized sport. Interviews were coded and analyzed for common themes relevant to experiences in organized sports. Items were reviewed following interviews and evaluated for consistency with experiences, as well as for wording of items.

The final step in Study One was to obtain evaluations from experts. Experts were recruited based on their understanding of sport participation and positive youth development to provide feedback on the items. This is an oft recommended step to ensure accurate interpretation (items are related to the construct) and coverage of a construct (e.g., not missing key aspects of the construct). Items were evaluated based on expert feedback and a final version of the ESSY produced for Study Two.

\section{Step One: Development of a Preliminary Scale}

The initial step was reviewing the previously discussed literature to develop a preliminary set of questionnaire items. This is termed a rational-theoretical approach to scale development and is a frequently employed method (Hubley \& Zumbo, 2013). Although a combined approach was ultimately used in the present study (e.g., interviews, expert-review), this was a good first step in the initial development of scale items. A 
rational-theoretical approach uses theory and logic to determine what should be included in the scale. The literature reviewed provides a strong background for including experiences of goal setting, adversity, and skill development in a measure of developmental experiences in sport. Supported by both theoretical and empirical work, each of these experiences provide important opportunities to promote positive developmental outcomes.

Using the information gathered and outlined previously in this document, a set of initial items was created and can be found in Appendix A. These items represent the constructs of goal setting, adversity, and skill development and as discussed, these items have been developed based on the idea that context, and exposure to different experiences within a given context, allow for developmental opportunities. These constructs represent valuable developmental experiences suggested by the literature and inherent to the sport environment.

\section{Step Two: Interviews and Qualitative Analysis}

The use of interviews and focus groups allowed for gathering of information from the intended survey respondents. For the present study, this refers to adolescents participating in organized sports or individuals who have participated in organized sports during their adolescence. Conducting interviews/focus groups has some important benefits. It allows for researchers to gather additional information, to synthesize information, and ensures that researchers are understanding how the construct is conceptualized from that perspective (DeVellis, 2017; Gehlbach \& Brinkworth, 2011). Specific to the development of the ESSY, it is necessary to understand how adolescents view their developmental experiences in sport and what features of their experience are 
salient. As such, the overarching goal of the interviews was to better understand how individuals who participated in organized sports viewed their experiences with sport.

Another aim of the interviews was to understand additional factors that were not found in the literature, such as positive and negative elements of sport participation (e.g., winning a championship or feeling pressure to perform). The purpose of interviews was not to be exhaustive but rather to gather information from enough sources until the point when no new information was presented (data saturation; Grady, 1998). Some suggest that ten participants should be sufficient (e.g., Simms, 2008). However, there is some discretion with this and is likely dependent on the quality of the interviews. For the present study, it was concluded that data was saturated after nine participants. This conclusion was based on the information obtained from the nine interviews and the fact that responses to interview questions were consistent, and frequently repetitive, across the participants. Furthermore, relatively little "new" data was being gained from each additional interview, indicating that saturation had been reached.

For the present study, to ensure that all potential factors were considered, a range of participants were recruited. This meant that adolescents were of importance, but adults who played organized sports during their adolescence were also consulted. This approach was taken with the expectation that it would yield additional information due to the retrospective aspect and a more holistic understanding of an adult's experience in organized sports as a youth.

The present study followed the recommendation of Gehlbach and Brinkworth (2011) and followed a two-step approach for the interviews. The first step was intended to allow the participant to express ideas, thoughts and opinions with little to no 
prompting. The second step allowed for more direct questions. A two-pronged approach gives the researcher the opportunity to obtain unbiased information while also being able to get more specific responses. The interview questions are presented in Appendix B. The first question asked for the participants' views on their own developmental experiences in sports. This was purposefully vague and lets the participant speak without influence from the interviewer. The questions after this were more probing and specific to the constructs established by the review of literature. The purpose of this part of the interviews was to ensure that scale items cover all angles of the constructs.

\section{Method}

\section{Participants}

A total of nine individuals were interviewed about their experiences playing organized sports. Demographic information, including pseudonyms, are provided in Table 1. Participants were recruited using convenience sampling and snowballing.

\section{Procedure}

Prospective participants and parents of prospective participants (i.e., those participants who are under the age of 18) were contacted via email and informed about the nature and purpose of the study. Interested parties were provided with a consent form to sign (see Appendix C). Parents were required to sign the consent form to allow their children to participate (see Appendix D). Participants 18 years of age or older did not require parental permission to participate. Once permission was obtained, participants were contacted, and a meeting time arranged. Interviews took place at the participant's home or other mutually agreed upon location and were recorded on an electronic recording device. 


\section{Table 1}

Demographic Information for Interview Participants

\begin{tabular}{|c|c|c|c|c|}
\hline Pseudonym & Gender & Age & $\begin{array}{c}\text { Years Playing } \\
\text { Organized Sports }\end{array}$ & Organized Sports Played \\
\hline James & Male & 13 & 8 years & Hockey, lacrosse \\
\hline Jimmy & Male & 15 & 10 years & $\begin{array}{l}\text { Soccer, hockey, ultimate frisbee, } \\
\text { golf, touch football, volleyball, } \\
\text { cross country, track and field }\end{array}$ \\
\hline Charli & Female & 16 & 10 years & $\begin{array}{l}\text { Soccer, badminton, track and } \\
\text { field, ultimate frisbee }\end{array}$ \\
\hline Ryan & Male & 21 & 15 years & Hockey, soccer, lacrosse, \\
\hline Kelsey & Female & 22 & 17 years & Hockey, ringette, track and field \\
\hline Ricky & Male & 38 & 18 years & Basketball, baseball, golf \\
\hline Tukey & Female & 31 & 6 years & $\begin{array}{c}\text { Track and field, volleyball, figure } \\
\text { skating, }\end{array}$ \\
\hline Lisa & Female & 35 & 11 years & Tennis, golf, hockey \\
\hline Lou & Female & 28 & 8 years & Hockey, track and field \\
\hline
\end{tabular}

\section{Interview Format}

As mentioned, a two-pronged approach was used for the interview process. Open ended questions were used in the beginning followed by more direct, probing questions. An iterative approach to the interview process was also taken with the initial set of questions altered as new information was presented. For example, one of the first participants interviewed described not feeling like they were able to work on their weaker skills during organized practice and, as skill development was expected to be an important experience, a question was added to specifically address this in future interviews. The final version of the interview protocol is presented in Appendix E. The interviews ranged between 15 minutes and 45 minutes with an average of 35 minutes. All interviews were transcribed into a Word document prior to analysis.

\section{Analysis of Qualitative Data}

Analysis of the qualitative data followed a direct content analysis approach. This 
type of analysis is one of three methods that fall under the umbrella of qualitative content analysis and allows for prior theoretical or empirical knowledge to guide interpretation of data (Hsieh \& Shannon, 2005). This method has been identified as useful in research studies where the objective is to validate or expand the understanding of a theory or framework (Zhang \& Wildemuth, 2017). It also allows for both an inductive and deductive approach to data analysis, which, some have argued, represents a more rigorous methodology (Fereday \& Muir-Cochrane, 2006).

Consistent with other theories of qualitative analysis (e.g., grounded theory; Charmaz, 2006), the first step was to conduct a line-by-line analysis. Initial coding began with identifying words and phrases consistent with goal setting, skill development, and adversity. Following this, an inductive approach was taken by examining words and phrases that were not consistent with goal setting, skill development, or adversity and assigning a code to ascertain if other patterns of experiences emerged from the data (Zhang \& Wildemuth, 2017). Coding began using transcripts from the first two interviews to develop an initial set of codes and new data was compared to support established codes or to expand understanding through development of new codes (i.e., constant comparative method; Charmaz, 2014). Findings from these analyses are presented in the following section.

\section{Results}

\section{Developing Skill and Being Skilled}

The development of competence is a key feature of PYD theory. Sport offers the opportunity for the development of competence through skill development. Primarily, this speaks to physical skill and the acquisition and development of sport-specific skills. 
These physical skills were important, but participants also spoke about skills that extended beyond those specific to the sport to include things like social skills and lifeskills (e.g., time management). Evidently, the development of skills was an essential feature of playing sports for these participants.

Participants spoke about feeling good about their ability and enjoying the feeling of performing well or making a good play. Being good at a sport was associated with feelings of confidence and many enjoyed the experience of being good at a sport. As Jimmy stated, “...first of all playing hockey gave me a sense of confidence. Before I played sports, I doubted myself and I eventually got good at sports and I had more confidence in daily things and overall sports." This sense of being good at something, in this case hockey, gave Jimmy confidence in other areas. All participants recognized that being skilled in a sport and the feeling that comes with being good at something was an important part of their sport experience.

Being good at a sport was also associated with the level of enjoyment of a sport. As Lisa succinctly stated, "I think the ones I was better at I enjoyed more." This was not an uncommon response to questions about why someone picked a sport as their favourite. For example, when asked why hockey was his favourite sport, Jimmy replied, "being good at it." When Ricky was asked about his favourite sport (baseball, basketball, or golf) he explained that it had changed through his adolescence and typically, his favourite sport was also the sport in which he was most skilled. It is not clear whether enjoyment encourages skill development, thereby enhancing skill or vice versa, but competence, and being skilled at sport, is a component of the sport experience.

Based on responses from participants, it was evident that skill development also 
extended beyond physical skill to social, psychological, and life skills. As Kelsey explains,

I would say just the physical activity piece is important but I'd say also developing like social skills, collaboration skills, cooperation, team building skills, all of that sort of thing I would say definitely I'd develop through sport. Sport was also an important context in which to learn how to deal with anxiety and stress. Participants described how learning to deal with anxiety before a competition or game often transferred to other areas of their life, such as taking tests or giving presentations. Many talked about being nervous when they played sports but that this was not always a negative emotion. For example, in describing how she felt sports benefitted her, Tukey described how being nervous helped her to develop better coping skills that helped her in other parts of her life,

Definitely physically, that's how I learned to get into shape or how to maintain body weight. But I also benefitted, you get better at managing anxiety as you do it. So even though I would get nervous to compete, I still found it helped my anxiety when I go to do something else. So whether it's writing a test now or, I found those skills kind of transferred over.

Most of the participants played at both a recreational and competitive level. An important noted difference was the focus on improving skill in competitive sports. With a heavy focus on winning, ensuring that athletes are developing and performing to the best of their ability is important. Ricky enjoyed his competitive experience more for this reason and the feeling that there was more purpose in playing at a competitive level, "recreation sports is what our parents put us in and we just run around for a little bit until 
we get to go home to do something else. In competitive sports it was about everyone wanted to get better individually and better as a team." This was echoed by Lou who felt that playing at a higher skill level with other skilled players was more enjoyable and pushed her to become a better player.

I like to be good at things and if I'm not good, I want to get better. In competitive sports I think you get more coaching, like, better coaching and that helps you get better. I don't think I would have developed my skills as well as I did if I hadn't played competitive sports and been in that environment where skill is important.

\section{The Experience of Adversity}

When specifically asked about facing adversity, it was not always easy for participants to recall situations in which they felt that they had experienced adversity. However, in assessing some of the experiences and responses to other questions, it is clear that participants had experienced some aspect of adversity, or faced challenges, and had developed strategies to overcome these challenges in sport. For example, Lou spoke about an experience in which her weakness in a specific skill was highlighted during a drill in a hockey camp,

I struggled a bit with skating backwards, like cross-overs and pivoting and we were doing these one-on-one drills where people were just blowing by me. I was really embarrassed and actually didn't go back because I didn’t feel like I, you know, belonged there. But, after that, I made a real effort during practices to really work on my backwards skating and I got to the point I could keep up with anybody.

Although this was brought up in discussions about skill development, it is an example of 
facing a challenge and finding a way to address and overcome the issue.

The challenge of having to overcome an obstacle meant working harder and putting in additional effort to improve a skill, cope with an injury, or accept a defeat. James put it the most succinctly, "If I miss a shot, I'll try really hard next time." For some participants, facing a challenge was motivation to improve. A few participants had reported being cut from teams and that this was a challenge to accept but ultimately forced them to work harder. For some, this experience ended up being beneficial to their overall experience, whether from a motivational standpoint or simply enjoying the team with which they ended up playing. For Ryan, being cut from a team that he felt he deserved to be on helped him to focus more on his own play and not be as concerned with how others evaluate him. Kelsey described feeling devastated by being cut from a competitive team but that she became a leader on the recreational team. This gave her an opportunity to develop her leadership skills that she would not have had otherwise. As she admits, it was very challenging to accept being cut from the team but it ended up being a really enjoyable season that "worked out."

Facing adversity also helped participants put things in perspective and prepare for the "real world". For Jimmy, losing back to back championship games helped him to learn an important life lesson, "coping with defeat and not being a sore loser because sometimes in your life you will go over a rough patch and you know you will overcome that." Kelsey had the most tangible experience with a severe injury and spoke about how facing this challenge helped her succeed,

I would say it probably helped a little bit like even in terms of building coping strategies, finding different ways to achieve goals whether that be like finishing 
an assignment or something like that I think sort of kind of working through, like, what limitations I had, probably it helped with that.

Having to work through her injury was an important part of developing strategies that helped her persevere, and not just in sport. Another common example is that of being nervous before a competition or game. Facing these nerves and having to deal with them was a fairly universal experience and one that helped participants learn ways to deal with feeling nervous (e.g., focus on other things, breathing exercises). Adversity was not something that everyone readily admitted to facing but through the discussion it became clear that there were adverse situations in each participants' experience with sport. The nature of the situation may have varied from missing shots or losing games to being cut from a team or having a serious injury. However, each situation led to a change in attitude or behaviour that produced a different outcome. In many cases, this outcome was positive and suggests that the individual experienced personal growth.

\section{The Importance of Social Relationships}

Perhaps the most salient theme discussed by all participants was the importance of social relationships within the sport context. All participants spoke to the value and significance of social relationships to their sport experience. This ranged from the sport as a context for developing and maintaining friendships, to relationships as a motivating factor or reason for playing sports, to the value of relationships between the individual and their coach and teammates. Consistent with Holt et al.'s conceptualization of PYD, social relationships were an essential feature of the participants' sport experiences.

\section{Coaches}

Relationships with coaches were prominent features of participating in sports. For 
most participants, coaches were a central figure in shaping the sport experience. This is wholly in-line with the model of PYD in sport but also takes it a step further to show how the relationship can shape the experience an athlete has in their sport. When a participant had a good relationship with a coach, this served to enhance the experience. Conversely, when a participant had a negative experience with their coach, the sport experience became similarly negative. It is interesting to note the extent to which some of these negative interactions with coaches stuck with the participants. For example, Lisa recalled an experience when a coach commented on her appearance telling her that if she lost weight, she would be a better player. Lisa discussed how difficult this was for her to hear as an adolescent who struggled with her body image and from an adult who had no real reason to make this judgement. From a more general perspective, Charli described how one of her coaches would frequently yell at her team and was quite aggressive in his demeanor during games. This affected how she felt about playing and that she would dread having to play when game day came around.

Negative interactions with coaches or a negative coach climate were quite salient for many participants. In fact, a negative interaction with at least one coach was a universal experience for each participant. The degree of negativity varied but there was always one coach who made the sport experience less enjoyable for the athlete. Some negative behaviour described by participants included a time when Ricky disagreed with a head coach who wanted to bend the rules in order to win a game when his baseball team was down by a run but time was running out to start a new inning. As Ricky states it, He changed his watch back and went up to the ump. I was thinking this isn't right. He thought he was doing what was best. I was 15 and I remember thinking this 
isn't right. That one stuck with me. There's no point in cheating to win. There's nothing to be gained from that.

Ricky's situation represents a challenging conundrum for an adolescent when a coach decides to do something he believes his best for the team but is counter to the spirit of the game. For Ricky, it was an impactful event that has stuck with him into adulthood. In another circumstance, this type of behaviour could have instilled a belief that cheating in sport is acceptable. In another situation, Kelsey describes how a coach impacted the team environment by his behaviour and seeming lack of preparedness and willingness to be a coach,

He didn't have a very extensive knowledge of ringette, just a quiet, laid back personality. He didn't take charge in handling any sort of team situations, was never prepared... he just kind of brought a negative team environment on. A lot of girls didn't like him so it just caused a lot of problem and tension on the bench. As can be seen in this reflection by Kelsey, a coach's behaviour can have a significant impact on the experience of team members and can sour a team dynamic. This is quite important as this is the leader of the group and an individual who has a lot of control in this situation. To that point, Charli expressed the following in describing a coach who would regularly get angry at the team, "he was insane, he would smash his clipboard and get really, really angry all the time and that's why I would, like Saturday would roll around and it would be futsal and I would dread going because of him." The coach's behaviour created such a negative experience that Charli, who loves soccer, dreaded playing.

Of course, positive interactions with coaches were also important for participants. 
Some features of a positive coach included being supportive and attentive of the player's needs. There seemed to be a sense on the part of the player that the coach understood them and cared about them. For participants, having a coach who really knew the sport was an important part of the positive climate. If the coach was able to foster their skill development and the team's performance as well as maintain a good relationship with the athletes, this was indicative of strong coaching. For Ricky, having energetic coaches who focused on fun was important for his recreational experience, but he was then grateful to have coaches who focused on getting the best out of their athletes at the competitive level. For Ricky, knowing the difference is a key part of successful coaching.

This idea of perceptive coaching and knowing how to deal with players and the team was mentioned by most participants. In some cases, getting the most out of a player meant recognizing the types of interactions that players needed at any given time. Ryan recognized this aspect, and explained,

Coaches are really important for the team. The thing is that they set the atmosphere for the team and they kind of, not decide, but have a great part of whether you like the sport or not. My coaches in B (level hockey) were awesome, all three were amazing and they all pushed me to be better and while still understanding that I sometimes, I knew that I had messed up, and they knew not to say anything.

Many attributed good coaching to their own success. For example, Lou said the following when describing her experiences starting out in hockey, I think I was lucky my first years playing hockey. It was awhile ago but I just remember it being good. He was, like, a nice guy but also, like, big on the 
fundamentals. And whatever he did just worked with me and I went and played on the competitive team the next year. I think I owe a lot of my success to that one coach.

Attributing success to coaches was frequently mentioned, as was being grateful for having a certain coach. Often, a positive experience in sport was punctuated by these positive relationships with coaches. Ultimately, coaches play a significant role in creating the sport climate and shaping the experiences that many have in sport. Although coaches are generally recognized as being important, perhaps the true extent of their influence and impact on youth is not yet fully appreciated.

\section{Teammates and Peers}

Participants spoke to the importance of developing and maintaining social relationships while engaging in sports. Again, this is consistent with the model of PYD in sport in that these relationships helped shape the experiences these participants had in sport. For a few, namely Tukey and Lisa, this was the very reason they played sports and why they continue to play sports. Without the social aspect, they both expressed uncertainty about whether they would have continued to participate in their respective sports. When answering the question about what comes to mind when you think about playing sports, Kelsey stated, "they're a lot of fun they bring a lot of joy to my life and then I've made a lot of friends through it so just being able to spend time with people, develop relationships, things like that." For Kelsey, relationships were at the core of what made playing sports enjoyable for her.

Many, if not all participants, spoke to the fact that teammates became friends and sport provided another context in which they could interact with friends, develop new 
friendships, and create stronger bonds. This speaks to how teammates can create a positive climate in which to develop, as suggested by Holt et al., (2017). Teammates enhanced the experiences, but the sport context also enhanced the relationships and created stronger friendships. As Charli stated, "I just love the feeling of being with my team. There's just a sense of unity and I love it so much...I love all of them and I'm friends with all of them and we're really tight..." When asked to explain why his favourite sport was lacrosse, James stated that "I've met a lot of people in lacrosse, it's just a really fun game and I really like it." Social relationships appear to be a core aspect of sport.

This was taken a step further by Ryan, who felt it was the support from his teammates that was important,

I have guys on my team that I know if someone hits me or runs me they'll step up for me. You have a full team in front of you that wants you to succeed and off the ice they're still your friends. (It's) nice having that. Trying to win together and after the game sit down and have a beer, talk, watch a game.

This was a consistent message from the participants - that sport provided a context in which teammates supported each other. Often, this support was in pursuit of a common goal, such as winning. Furthermore, participants talked about how encouraging teammates were. If a mistake was made or someone was having a bad game, often teammates were a source of comfort or inspiration to keep trying. Teammates can help overcome a challenge by providing that sense of support. Again, this harkens back to the sport-based model of PYD and how teammates create the climate in which positive development occurs. 
There were some negative interactions with teammates and peers. These negative interactions generally took the form of team members not getting along or having "cliques" on the team. That said, such experiences were not particularly salient and often dismissed. Some participants stated that they simply chose not to engage in such behaviour while others attributed it to normal adolescent behaviour. It was acknowledged that this behaviour was unpleasant, wrong, and not enjoyable but it did not necessarily impact the overall experience. It seemed to be viewed more as an element of peer relations as opposed to a feature of their sport experience.

\section{Limited use of Goal Setting Strategies}

As discussed in the review of the literature, goal setting has many implications for outcomes in youth. Based on the extant literature regarding the value and importance of goal setting in sports, it was expected that this would be a key feature of the sport experience. Most participants did have goals but the act of goal setting or processes associated with achieving goals were not salient experiences. Participants who did identify goals were typically speaking about goals that were very general in nature. Some goals were based on outcome measures, such as James, "score three goals in the next 5 games, or something like that" while others were focused on improvement. That was the most commonly stated goal - that the athlete just wanted to improve in their sport. For example, Charli stated,

I mean I always want myself to be better. I always try to improve whatever I can.

One of the bigger ones is just keeping in shape so I will run more and like do more training and like working out training so I can be better in my games and not get as tired. 
Or as Ricky described, "I didn't sit down and write down what I want to accomplish. I just wanted to work my butt off and get better at it." In each of these statements, there is an implicit goal driving behaviour, which is the part that is important when considering PYD theory and the importance of goal setting. Even though it does not look like what it is supposed to (according to goal setting literature), goals are driving behaviour. Participants want to get better and are motivated to work to achieve that goal.

All participants felt that they had achieved goals in sports. That is another important aspect to bear in mind. When the question was asked if they felt they had achieved goals, everyone responded enthusiastically and confidently that they had. Even though not many had engaged in rigorous goal setting, all participants felt that they had been successful in some aspect of their sport. The nature of participation for each participant may have played a role in why the experiences are not congruent with what the literature states. Much of the work conducted under the sport psychology umbrella is done with elite athletes and their experiences are likely different than athletes at lower levels of competition.

Two participants did describe engaging in the level of goal setting generally recommended in the sport psychology literature. Tukey and Ryan were involved in sports where a one-on-one coach was available. Tukey participated in figure skating and described how they would develop their goals for the year,

So at the beginning of the year you would say, I want to pass the waltz and I want to pass this tango dance. And that's what I'm going to spend my time on. And it was the same thing for free skate, it was the same levels. So you'd make like concrete goals like that. It started with jumps, you'd really want to land the axel, 
or I want to compete at this many competition. So that was always done. With volleyball and track I don't remember goal setting.

Ryan was a goalie with a goalie coach who encouraged him to develop goals, write them down and consistently reflect on these goals and how to achieve them. Both of these participants had this unique opportunity of one-on-one coaching that may explain why the other participants did not have the same goal setting strategies.

Despite the focus on goal setting as a key element of positive youth development, the experience of participants did not align with these expectations. As mentioned, participants had goals and felt that they had achieved goals in sport but only a couple had experience with goal setting strategies that are said to be important parts of the goal setting process.

\section{Importance of Sport}

For all participants, sport was an important part of their lives in some capacity. For each, there was at least one aspect of sport that made it a key part of their daily or weekly lives. For example, Lisa and Tukey felt that the social aspect of sport was what drew them to participate and to continue to participate. For a number, the physical component and the opportunity to maintain an active lifestyle was a very strong motivating factor. For a couple, it was as simple as they just loved playing sports. Charli felt that sport helped to shape who she was, and it was a part of her that she wanted people to recognize. Most participants identified as an athlete and described sport as playing a central role in their own identity, I've always described myself as an athlete. Like anytime I was asked to introduce myself or, you know, what are 5 words you would use to describe yourself, 
athlete would always be one of them. It was absolutely an important part of who I was growing up. And, I mean, I don't really play any organized sports anymore but I'm still, like, pretty active and I definitely still think of myself as an athlete. Interestingly, Lisa did not feel that she was an athlete, but she acknowledged that playing sports and being active was an important part of who she was and who she is.

I also think as a teenager I had weight problems here and there and I needed sports. I didn't realize it at the time but it kickstarted my lifestyle. I need to move and be active. I feel good when I am active, and I think it's because I've always been active and played sports and I still need to be.

For the most part, participants felt that sport was a large time commitment but that this commitment was worthwhile. No one felt that they were missing out on other things by participating in their sports. Many felt that anything missed was made up for by the fact that they were engaging in something that they loved. It should be stated that everyone enjoyed playing sports. Some had different motivations or levels of engagement (e.g., social aspects) but all reported some level of investment and engagement in their sport (or sports).

A few participants described how they looked forward to playing sports and the fun they had while playing sports. Ryan felt that playing hockey was something that would be a part of his life for as long as possible because of the excitement he felt whenever he plays.

I never have to force myself to do it - to do a run I have to force myself out of bed to go do it. I'm excited to go to the rink put on my gear go on the ice. It's a part of my life that will always stay there and I will be doing it for as long as I can. I 
enjoyed it growing up and still enjoy it now.

Ryan also mentioned the calming effect that playing sports had on him and how he could count on sports to provide a relief from the stress of life. That was echoed by a few participants who felt that sport could provide a temporary escape into something that was purely enjoyable.

Ultimately, playing sports was a meaningful and significant part of participants' lives with many stating they would continue to play sports for as long as they could. Some suggested that the type of involvement might change. For Kelsey and Ricky, coaching was something they expressed interest in to maintain a connection with the sports they loved. For others, it was participating in a more recreational way, like Tukey and Lisa who were more invested in the social aspect of sports.

\section{Discussion}

The overarching purpose of conducting interviews was to better understand the experiences that individuals have when playing sports during adolescence. It was expected that this process would yield additional information to add to the development of a measure of youth experiences in sport. The interviews did provide some aspects to consider for adding items to the measure and/or modifying existing items. However, the key component to come out of these interviews was a confirmation of the model proposed by Holt et al., (2017) and the fact that coaches and peers shape the sport experience. The nature of these relationships significantly impact experiences in sports and the outcomes of these interactions with coaches can be long lasting. This is particularly true when there is a negative interaction with a coach. The power and influence of these relationships within the context of sport cannot be understated. Holt et 
al., suggested that coaches and teammates created the climate in which PYD occurs. The results from participant interviews takes this a step further to suggest that relationships create the climate but also further shape the experience and may hinder or enhance the experiences that foster PYD.

Another component to consider is the importance placed on playing sports. The enjoyment and value placed on sports was a key reason as to why many participated in organized sports and often what led participants to continue playing beyond adolescence. Research has clearly demonstrated an association between enjoyment in sport and outcomes such as continued participation (Gardner et al., 2017) and commitment (Weiss et al., 2001). However, this may go beyond mere enjoyment to a more critical aspect of placing a high level of importance on sports, whether that is to one's identity, social life, or means of being physically active. Most of the participants felt that sport was part of who they were and that sports would always be a part of their lives in some way. As an example, Kelsey could no longer play ringette due to injury but stated that she would become a coach to ensure that she could remain connected with the sport.

Consistent with the literature, skill development and perceived competence were important parts of the sport experience. Feeling good about their ability and having confidence in their physical skill was a common sentiment, as was the idea of favouring a sport because of being good at that sport. A few participants talked about how skills learned while playing sports were transferred to other areas of their lives. This was a common sentiment around social skills and coping with anxiety. For example, participants spoke about experiencing anxiety before a big game or competition and learning techniques to reduce this feeling and how these techniques then helped them 
when taking tests or exams. It is through this experience in sports that athletes are able to gain confidence in their skillset and apply that skillset to other areas of life.

The transfer of skill is outlined in Holt et al.'s model under the explicit pathway and has been studied by a number of researchers (i.e., life skill transfer; Bean et al., 2016; Newman, 2020). The focus of the present study was on the implicit pathway and experiences closely tied to the sport environment. Physical skill and perceived competence were salient features of participants' experiences with sport. In addition, experiences align with developmental theory of the opportunity to develop and display competence as a significant feature of PYD environments. The transfer of skills is important but speaks to the outcomes associated with the experiences associated with skill development and facing adversity (e.g., feeling anxious before a competition).

Participants reported a variety of experiences with adversity. Examples included overcoming a skill deficit, being cut from a team, and facing a serious injury. Many perceived these experiences as beneficial to their overall experience. While at first, they may have seemed challenging and were emotionally difficult to overcome, in the long term most felt that the experience helped them grow (e.g., improve their skill, feel more confident) and learn (e.g., leadership skills, coping skills). Facing challenges in sport was universally experienced and the benefits are consistent with the literature. For example, (Galli \& Reed, 2012) found that athletes had higher self-confidence after facing adversity and this was one benefit reported by Ryan after being cut from a competitive team. Furthermore, Kelsey described being devastated after being cut from a team but then having one of her best years in sports. This is consistent with findings from (Wadey et al., 2013) who argued that overcoming adverse situations can actually lead to greater 
enjoyment in sport.

Given the importance placed on goal setting in much of the literature, it was surprising to hear from so many participants that they did not engage in many of the goal setting practices. Both the PYD and sport-based literature have spoken to the value of youth engaging in goal setting to encourage positive outcomes and behaviours. As stated, all participants had goals, but few used specific strategies, such as outlining process, performance, and outcome goals. Two of the participants who had one-on-one coaching experiences did have experiences with goal setting that included many of the strategies outlined in the goal setting literature. This may highlight an important aspect to assess when evaluating youth experiences in sport - the type and level of sport participation. Perhaps those in individual and/or higher level competitive sports will experience goal setting differently than those in team or lower level sports.

The interviews were beneficial in confirming the model proposed by Holt et al., (2017) and in providing support for the development of a measure to assess experiences in sport. Experiences reported by participants were consistent with what was outlined by the literature in terms of skill development, facing adversity, and goal setting. There is an evident need to consider relationships within the sport context and how these relationships impact the sport experience. This takes what has been proposed by Holt et al., a step further to include the potential for the PYD climate factors to moderate the association between experiences in sport and PYD outcomes.

With the perception that coaches and teammates played a significant role in shaping many of the participants' experiences, a secondary model was proposed for testing in Study 3. This model tested the interaction between relationships in the sport 
context and experiences and tested the assertation made by most participants that their experiences were influenced by their relationships with teammates and coaches.

\section{Step Three: Expert Validation of the ESSY}

Based on the findings from the analysis of the interviews, the ESSY was updated to reflect the nature of the experiences reported by the interview participants. Significantly reducing the goal setting items was considered to make the items more congruent with the interview participant's experiences. However, based on the importance of goal setting to developmental outcomes, as described by the literature, it was decided that most items should remain. The items for the skill development, adversity, and goal setting construct are presented in Table 2.

In line with what was expressed by interview participants, a new construct was added to the ESSY. A total of seven items relating to Sport Climate were added to capture the importance placed on coach and teammate relationships as well as the importance of playing organized sports. These new items are presented in Table 3. Following the interviews, a specific definition was developed for each of the factors within the scale. Clearly defining each construct is important for identifying what it is you are intending to measure and ensuring that it is accurately conveyed through the items (Clark \& Watson, 2016). Construct definitions are presented in Table 4.

The response format of the ESSY is a 5-point likert scale. To be considered a continuous variable, experts recommend using five response categories (Bandalos \& Gerstner, 2016; Rhemtulla et al., 2012). Respondents are given the prompt to think about their experiences in sport and to respond to how closely the item describes them and their experiences. The response options range from Never (1) to Always (5). The option of 
agree versus disagree responses was not used based on the recommendation by Gehlbach

and Brinkworth (2011) who state that agree versus disagree response options increase cognitive load on participants and increase the potential for error.

\section{Table 2}

Preliminary items for the Experience Sport Scale for Youth

\begin{tabular}{|c|c|c|}
\hline Construct & $\begin{array}{l}\text { Item } \\
\text { Number }\end{array}$ & Item Description \\
\hline \multirow[t]{10}{*}{ Goal Setting } & ESSY1 & I have goals. \\
\hline & ESSY2 & I set my own goals. \\
\hline & ESSY3 & I have achieved goals. \\
\hline & ESSY4 & I set goals with my group/team. \\
\hline & ESSY5 & I have worked hard to achieve a goal. \\
\hline & ESSY6 & I set long term goals. \\
\hline & ESSY7 & I set short term goals. \\
\hline & ESSY8 & I have specific plans/steps to achieve my goal \\
\hline & ESSY9 & I have told others about my goals. \\
\hline & ESSY10 & I regularly reviewed my goals. \\
\hline \multirow[t]{9}{*}{ Adversity } & ESSY11 & $\begin{array}{l}\text { I have faced challenges (for example, cut from a } \\
\text { team, suffered an injury, had to sit out games). }\end{array}$ \\
\hline & ESSY12 & I have lost an important game/competition. \\
\hline & ESSY13 & I have struggled to learn a new skill. \\
\hline & ESSY14 & $\begin{array}{l}\text { I have played on a team where I have not gotten } \\
\text { along with some people. }\end{array}$ \\
\hline & ESSY15 & I have disliked one of my coaches. \\
\hline & ESSY16 & I have made sacrifices to help me or my team win. \\
\hline & ESSY17 & I have made a mistake that cost me or my team. \\
\hline & ESSY18 & I have felt nervous before a game/competition. \\
\hline & ESSY19 & $\begin{array}{l}\text { I am afraid to make mistakes during a } \\
\text { game/competition. }\end{array}$ \\
\hline \multirow{8}{*}{$\begin{array}{l}\text { Skill } \\
\text { Development }\end{array}$} & ESSY20 & I have had an opportunity to develop new skills. \\
\hline & ESS721 & I have worked hard to improve a specific skill. \\
\hline & ESSY22 & I have developed a wide range of physical skills. \\
\hline & ESSY23 & I had help from others to improve skills. \\
\hline & ESSY24 & I have worked on skills on my own time. \\
\hline & ESSY25 & I am skilled in my sport. \\
\hline & ESSY26 & I put a lot of effort into developing my skills. \\
\hline & ESSY27 & $\begin{array}{l}\text { If I am not good at a skill, I work harder on that } \\
\text { skill. }\end{array}$ \\
\hline
\end{tabular}




\section{Table 3}

Items Representing Sport Climate

\begin{tabular}{ccc}
\hline Construct & Item Number & Item Description \\
\hline Sport Climate & ESSY33 & I play sports because I enjoy it. \\
& ESSY34 & I am friends with my teammates. \\
& ESSY35 & I have a good relationship with my coach. \\
ESSY36 & My coach gets angry when I make a mistake. \\
& ESSY37 & My teammates get angry with me when I make \\
& ESSY38 & a mistake. \\
& ESSY39 & Playing sports is an important part of who I am. \\
\hline
\end{tabular}

Table 4

Definitions of Constructs for the ESSY

Construct
Goal Setting
$\begin{gathered}\text { The identification of a specific sport-related objective that } \\ \text { focuses effort and motivates the individual. The goal setting } \\ \text { questions are designed to assess whether or not participants set } \\ \text { goals, the general nature of these goals and if they felt like they } \\ \text { had achieved them. }\end{gathered}$
Tdversity
$\begin{gathered}\text { The experience of a challenging situation in the sport } \\ \text { environment. The adversity questions are designed to assess if } \\ \text { participants feel like they have faced adversity and if they have } \\ \text { experienced different types of common adverse situations in }\end{gathered}$
sport.
Thill
Development
The acquisition of physical skills in sport. The skill development
opportunity to develop skills and if they were self-
directed/motivated in some of their skill development.
The general attitude that individuals have toward sport and their
sport environment. The sport climate questions are designed to
assess the motivation for playing sports, the importance placed on
sports, and the perceived climate of peer and coach relationships
in the sport context.




\section{Expert validation}

Having subject-matter experts evaluate a proposed measure is a consistently recommended step in scale development procedures (Simms, 2008; Worthington \& Whittaker, 2006). For the evaluation stage, two different approaches were taken to gather expert opinion on the proposed measure. The first stage involved providing two experts with copies of a selection of transcripts and asking them each to review the transcripts for major themes and then assess the items included in the ESSY. This was done to ensure a breadth of understanding and that participants' experiences were accurately interpreted.

The second stage involved giving four experts a copy of the measure with all potential items along with an evaluation sheet (see Appendix F). The experts consisted of individuals who were proficient in developmental psychology, positive youth development, scale development and/or involved in youth sports. Each expert was asked to evaluate each item for clarity, relevance, etc. and to provide input on any aspect that may be missing, irrelevant, or unclear. The main goal of this step was to ensure content was appropriate and representative of the construct being measured. To be consistent with the purposes of the present study, experts were considered adults who had been involved in coaching youth sports and graduate-level students studying in the area of positive youth development.

Experts pointed out that some of the items in the initial Sport Climate subscale were related to relationships while others were related to an identity construct. It was suggested that these be separated to create a Relationship Quality in Sports subscale and a Sport Identity subscale. The decisions regarding this feedback are discussed below. Based on feedback from experts and feedback during the interviews, the word 'adversity' 
was changed to 'challenges' in one item to read I have faced challenges in sports. Other than those suggestions, the reviewers felt that the items accurately reflected the constructs. As a final step, each item of the ESSY was assessed for reading level to ensure that the target audience (i.e., adolescence) would be able to read and understand the items. According to the Flesch-Kincaid grade level indicator provided by Microsoft Word, each item was at a grade 4 level of reading.

Upon reflection, relationships aspects are covered in the sport climate construct of the Sport-based model of PYD. They are not "experiences" but influential features of the sport environment. This was identified by the Holt et al., model and supported by the qualitative interviews. As the purpose of the ESSY is to identify salient experiences within organized sports, questions about relationship quality were deemed not necessary or appropriate for the questionnaire. For this reason, questions pertaining to the coach and/or teammate relationships were removed. Similarly, sport identity does not speak to what is being experienced in a sport setting. It may be an important part of the sport experience but is not, in and of itself, an experience. Questions pertaining to sport identity were also removed. A critique of previous attempts at creating a sport experience survey was the inclusion of items related to both process and outcome factors. The ESSY is very much intended to focus on experiences and assessing that process component of the Sport-based Model (i.e., answering how sport promotes PYD). Therefore, it was decided that the items consistent with this aim would be retained.

\section{Summary}

Although not the primary aim, Study One confirmed that the sport-based model of PYD is accurate in how it posits that relationships create the sport climate to produce 
positive developmental outcomes. However, the interview findings provided support for the inclusion of an experiential factor in sport and to examine relationships as moderating factors in the association between experiences and PYD outcomes. To that point, interviews further supported the primary aim of Study One to develop a measure of experiences in sport. Findings were consistent with the literature, with the exception of aspects of goal setting. Finally, expert validation provided additional support for the preliminary ESSY items and gave the opportunity to reflect on the purpose of the ESSY. Ultimately, the items presented in Table 2 represent the version of the ESSY to be tested in Study Two (ESSY v1.0).

\section{Study Two: Assessing Factor Structure}

With a set of items deemed to be an accurate representation of three key experiences in the sport setting, the factor structure was then assessed in Study Two. To assess the factor structure, the ESSY v1.0 was administered to individuals between the ages of 13 and 19 and the responses were analyzed using factor analysis. It was expected that items would load on their respective constructs and that the analyses would yield three separate factors of goal setting, adversity, and skill development.

\section{Data Collection and a Global Pandemic}

Data collection for this thesis was ongoing from 2019 to 2020 and was scheduled to take place in high schools during the winter of 2020. Unfortunately, the COVID-19 pandemic resulted in the closure of public institutions in most of Canada including Ontario and Nova Scotia, where data collection with high school students had been planned. The studies had to move forward with data that had already been collected and the majority had been collected from undergraduate students. 


\section{Method}

\section{Participants}

Participants for this study were $N=334$ male and female adolescents (13-19 years old, $M_{\text {age }}=17.36 ; 63.87 \%$ females) recruited from the community of a major central Canadian city and from undergraduate psychology courses of a central Canadian university. All of those who participated were required to have been engaged in an organized sport within the previous 12 months and have at least one year of experience in organized sports. As mentioned, the COVID-19 epidemic in Canada prevented further recruitment of adolescents in the community (e.g., high schools and sports teams). Because of this, the majority of the sample was from an undergraduate population.

\section{Procedure}

A secure online network was used to recruit participants and all measures were completed online using a personal device (i.e., computer, cell phone, tablet; see Appendix G for recruitment poster). Participants who accessed the study through this network received credit toward an introductory psychology course as compensation for their participation. All participants provided consent prior to accessing the survey (see Appendix $\mathrm{H}$ for consent form).

A few participants $(n=23)$ were recruited from local high schools. Approval to recruit from schools was granted by the school board and by the principal of the respective schools. Teachers of physical education classes and school sports teams were contacted by the principals and interested parties contacted the lead researcher. A time was then arranged to go into classrooms and hand out permission forms for parents to sign (see Appendix I). Data collection took place approximately a week later. Students 
had to have parental consent and voluntarily consent themselves to participate (see Appendix $\mathbf{J}$ for student consent form). All participants completed paper copies of the questionnaire and were provided a debriefing form with additional information about the study and resources should they need support following the study (see Appendix K for debriefing form).

\section{Measures}

All participants completed a background information questionnaire to provide information relating to age, gender, sports participation experience, type of sports played, etc. The full background questionnaire can be seen in Appendix L.

Experience in Sport Scale for Youth (ESSY). Each participant completed the 27-item version of the ESSY proposed from Study 1 (see Appendix M). Participants were prompted with "think about your experiences playing organized sports and how closely the items describe you and your experiences. In sport..." Participants then rated the frequency of experience with the item on a scale from 1 (never) to 5 (always).

\section{Results}

The purpose of Study 2 was to evaluate the association between factors as assessed by the observed variables, or items, proposed in the ESSY using exploratory factor analyses (EFA). Prior to conducting the analyses, the dataset was screened for missingness and to ensure assumptions of EFA were met. Preliminary data analyses were carried out using SPSS v26.0 and SAS v9.4. Sampling adequacy was confirmed using the Kaiser-Meyer-Olkin measure of sampling adequacy $(\mathrm{KMO}=.89)$.

\section{Missing Data}


Patterns of missingness were assessed visually as well as with a Missing Value Analysis. Overall, the proportion of missing data was low. Looking at the items, ESSY8 and ESSY10 were missing six responses; ESSY7, ESSY9, and ESSY26 were missing 3 responses; ESSY6 and ESSY27 were missing 2 responses. Remaining items were either missing a single data point or had no missingness. At the participant level, one participant was missing 19 responses and, after reviewing Mahalanbois Distance scores for careless responding (Meade \& Craig, 2012), this participant was removed. An additional 19 participants had missing values for one, two or three items and all remained in the dataset. Because the proportion of missingness was relatively low, listwise deletion was used to deal with missing values for the EFA.

\section{Assumptions of Exploratory Factor Analysis}

Similar to assumptions of regression, it is important to assess normality, linearity, collinearity, and the presence of outliers (Flora et al., 2012). This was primarily done through visual inspection of histograms, scatterplots, boxplots, as well as skewness and kurtosis values. Some items were found to be significantly, negatively skewed. Although it would be not be unexpected to have skewed values given that these individuals selfselected to participate in a study about experiences in sports, the distribution of a few of the items were concerning. Of note were ESSY1, ESSY18, and ESSY19 which had a significant number of scores piled on the upper limits. At this point, all items remained in the dataset and no action was taken to adjust the distribution. However, the information was used to make decisions regarding item selection and/or deletion during the EFA.

There was a linear association between the items within their respective constructs. Some items were only correlated with the items within their respective 
construct (e.g., My coach gets angry with me when I make a mistake and I have made a mistake that has cost me or my team) and did not show a linear association with many other items. No other significant issues were found related to collinearity or outliers. After assessing the data, it was determined that the data satisfactorily met the assumptions to continue with the EFA. As EFA is an iterative process, problem items would be removed and then the analysis performed again. For this reason, it was deemed to be more prudent to keep items and use information from the data cleaning step to help make decisions regarding the retention or deletion of said items during the EFA.

\section{Plan of Analyses: Exploratory Factor Analyses}

Exploratory factor analysis is used frequently in scale development to assess associations among items in a proposed scale. Specifically, exploratory factor analysis is used to assess patterns of correlations between scale items (Tabachnik \& Fidell, 2013). The analysis is particularly useful for determining if subsets of items (e.g., subscales) will group together in a meaningful way. Variables that group together, or correlate, represent the factors, or constructs, of a proposed scale. It is assumed that it is the underlying construct that is causing these items to group together, independently of other subsets (Tabachnik \& Fidell, 2013). Items that do not group or that are grouped on more than one factor can be assessed for deletion.

As discussed, each of the subscales of the ESSY are features of the sport environment and consistent with experiences in organized sports. As such, it was expected that there would be an association between the factors (i.e., factors would correlate) so an oblique rotation method was used (i.e., promax; Costello \& Osborne, 2005). With an oblique rotation, the pattern matrix was interpreted as it reports the unique 
contribution of the factors to the variance of each item (Tabachnik \& Fidell, 2013). Visual inspection of the structure matrix was also used to confirm interpretations of the pattern matrix. Factors were extracted using principle axis factoring (PAF). PAF was selected because it does not require that the data be normally distributed (unlike maximum likelihood; Fabrigar et al., 1999).

As mentioned, conducting an EFA is an iterative process whereby an EFA is run, items are evaluated and may be systematically removed, and then the analysis is run again. Evaluation of items for deletion involved the use of objective and subjective assessments. Primarily, factor loadings were assessed to see if item loadings on a factor were above the critical value of .35 and/or if any items were loading on more than one factor (Tabachnick \& Fidell, 2013). Items that had a loading below .35 were considered for deletion, as were items that loaded above .35 on more than one factor (Tabachnick \& Fidell, 2013). Communality scores were evaluated and any values below .40 were noted (Tabachnik \& Fidell, 2013). A low communality score can indicate that the item does not share much variance with other items. Finally, the meaningfulness of the item to the factor was also assessed and any items that reflected poor wording or an inaccurate representation of the specific construct were considered for deletion.

The number of factors to retain were, again, determined based on objective and subjective assessments. Eigenvalues represent the relative importance of a factor (Field, 2009). The larger an eigenvalue, the larger the variance associated with that factor (Tabachnik \& Fidell, 2013). One way to use eigenvalues to determine the number of factors to retain is to examine a scree plot. In a scree plot, eigenvalues are plotted on the $\mathrm{Y}$-axis and the associated factors are plotted on the X-axis. Any points above the point of 
inflexion are said to represent meaningful factors that should be retained. That said, most authors do not recommend basing decisions solely on a scree plot (e.g., Watkins, 2018). Another method to determine the number of factors to retain is to assess eigenvalues for each factor. It is generally accepted that eigenvalues above 1.0 are meaningful and can be retained (Field, 2009; Tabachnik \& Fidell, 2013). A final step that is often recommended with eigenvalues is to run a Monte Carlo parallel analysis. Each of these steps was taken in the present study to determine which factors to retain. As with retention of items, the meaningfulness of the factor was also considered before final decisions were made (Costello \& Osborne, 2005).

\section{Demographic Information}

Prior to completing the EFA, demographic and background information for the sample was assessed. The majority of participants (53.8\%) came from households where the mother had obtained a college or university level degree. Years participating in organized sport ranged from one to 16 with soccer (68\%) swimming (38\%), hockey (33.4\%), basketball (31.6\%), and dance (28\%) as the top five sports listed. Most participants did not engage in other extra-curricular activities, other than volunteering (60.5\%; note that volunteering hours are required for graduation from Ontario high schools). When answering survey questions, participants were asked to think about their experience in a specific sport and respond to whether that sport was competitive or recreational, and if they considered that sport to be their favourite. The majority of participants reported that the sport they would be thinking of was a competitive sport $(61.9 \%)$ and it was their favourite sport (85.5\%).

\section{Results of Exploratory Factor Analysis}


For this study, the EFA process was repeated eight times before satisfactory factor loadings and factor structure were obtained. The main problem each time was with items cross-loading or not loading on the expected factor. As described in the plan of analyses, problematic items were removed one at a time and then the EFA was performed again. Factor loadings from the first analysis are presented in Table 5 and suggest a 6-Factor solution. As can be seen in Table 5, ESSY4, ESSY6, and ESSY7 load on both Factor 1 and Factor 6. In addition, ESSY18 and ESSY19 loaded by themselves on Factor 5 while ESSY24, 26, and 27 loaded on Factor 3. Finally, ESSY25 failed to load on any factor. The eigenvalues did not support a six-factor solution (.48) nor did the assessment of the scree plot.

In the subsequent analyses, ESSY1, ESSY15, ESSY16, ESSY18, ESSY19, ESSY25, ESSY26, and ESSY27 were removed. For the most part, each of these items were removed because factor loadings were not satisfactory. However, ESSY1 was removed after considering its meaningfulness to the factor and the fact that the distribution of scores was negatively skewed. The piling up of responses suggests that the item is not capturing different levels of the construct (i.e., everyone has a goal). Reflecting on the wording of the item (I have goals) and the overall purpose of the factor (i.e., to assess goal setting), it was deemed to be a poor item and was removed. The final 19-item, three-factor solution is presented in Table 6.

As can be seen in Table 6, the 19-items loaded satisfactorily on the respective factors. Factor 1 represents the goal setting construct, Factor 2 represents the skill development construct and Factor 3 represents the adversity construct. Eigenvalues for the three factors were each above 1.00 and the scree plot showed a strong inflection point 
Table 5

Factor loadings from the First Analysis of the ESSY Factor Structure

\begin{tabular}{|c|c|c|c|c|c|c|c|}
\hline Item & Item Description & F 1 & F 2 & F 3 & F 4 & F 5 & F 6 \\
\hline 1 & I have goals & $.67 *$ & .11 & .13 & -.05 & -.01 & -.60 \\
\hline 2 & I set my own goals & $.66^{*}$ & .09 & .13 & -.04 & .02 & -.11 \\
\hline 3 & I have achieved goals & $.50^{*}$ & .05 & .12 & -.07 & -.10 & .13 \\
\hline 4 & I set goals with my group/team & $.47^{*}$ & .15 & -.15 & -.03 & -.01 & $.45^{*}$ \\
\hline 5 & I have worked hard to achieve a goal & $.58^{*}$ & .16 & .09 & -.09 & .07 & -.02 \\
\hline 6 & I set long term goals & $.51^{*}$ & .02 & .01 & -.02 & .12 & $.41 *$ \\
\hline 7 & I set short term goals & $.46^{*}$ & -.06 & .15 & -.06 & .14 & $.45^{*}$ \\
\hline 8 & $\begin{array}{l}\text { I have specific plans/steps to achieve } \\
\text { my goals }\end{array}$ & $.68^{*}$ & -.17 & .12 & -.03 & .08 & .20 \\
\hline 9 & I have told others about my goals & $.74^{*}$ & -.08 & -.17 & .12 & -.10 & .00 \\
\hline 10 & I regularly reviewed my goals & $.77 *$ & -.03 & -.07 & .12 & -.04 & .11 \\
\hline 11 & I have faced challenges & .12 & .11 & -.04 & $.48^{*}$ & .06 & .09 \\
\hline 12 & $\begin{array}{l}\text { I have lost an important } \\
\text { game/competition }\end{array}$ & -.02 & .15 & -.06 & $.56^{*}$ & .18 & .00 \\
\hline 13 & I have struggled to learn a new skill & .04 & .16 & -.08 & $.55^{*}$ & .15 & -.14 \\
\hline 14 & $\begin{array}{l}\text { I have played on a team where I have } \\
\text { not gotten along with some people }\end{array}$ & -.03 & -.08 & .12 & $.59^{*}$ & -.06 & -.11 \\
\hline 15 & I have disliked one of my coaches & -.20 & -.20 & .21 & $.44^{*}$ & .10 & .03 \\
\hline 16 & $\begin{array}{l}\text { I have made sacrifices to help me or } \\
\text { my team }\end{array}$ & .17 & .07 & .05 & $.38^{*}$ & -.16 & .03 \\
\hline 17 & $\begin{array}{l}\text { I have made a mistake that cost me } \\
\text { or my team }\end{array}$ & .03 & -.14 & -.04 & $.53^{*}$ & -.06 & .06 \\
\hline 18 & $\begin{array}{l}\text { I have felt nervous before a game or } \\
\text { competition }\end{array}$ & .10 & -.03 & .01 & .13 & $.61^{*}$ & -.04 \\
\hline 19 & $\begin{array}{l}\text { I am afraid to make mistakes during } \\
\text { a game or competition }\end{array}$ & -.08 & .03 & -.02 & .00 & $.63^{*}$ & .10 \\
\hline 20 & $\begin{array}{l}\text { I have had the opportunity to develop } \\
\text { new skills }\end{array}$ & -.04 & $.84^{*}$ & -.07 & -.02 & .03 & .05 \\
\hline 21 & $\begin{array}{l}\text { I have worked hard to improve a } \\
\text { specific skill }\end{array}$ & .02 & $.69 *$ & .17 & -.10 & .01 & -.01 \\
\hline 22 & $\begin{array}{l}\text { I have had the opportunity to develop } \\
\text { a wide range of physical skills }\end{array}$ & .01 & $.63 *$ & .14 & .03 & -.06 & .03 \\
\hline 23 & $\begin{array}{l}\text { I had a lot of help from others to } \\
\text { improve skills }\end{array}$ & .02 & $.49 *$ & .22 & .05 & .05 & .02 \\
\hline 24 & $\begin{array}{l}\text { I have worked on skills on my own } \\
\text { time }\end{array}$ & .07 & .02 & $.71^{*}$ & .03 & -.03 & -.10 \\
\hline 25 & I am skilled in my sport & .13 & .21 & .30 & .19 & -.23 & .22 \\
\hline 26 & $\begin{array}{l}\text { I put a lot of effort into developing } \\
\text { my skill }\end{array}$ & -.01 & .15 & $.75^{*}$ & -.01 & .03 & .03 \\
\hline 27 & $\begin{array}{l}\text { If I am not good at a skill, I work } \\
\text { harder on that skill }\end{array}$ & .01 & .05 & $.77^{*}$ & .01 & .03 & .03 \\
\hline
\end{tabular}

*denotes values above $.35 ; F=$ Factor 
at the 4-factor point indicating that three factors should be retained. Item loadings range from .45 (ESSY24) to .80 (ESSY21) and are all above the critical factor. This threefactor solution reportedly explained $102.65 \%$ of the variance. Looking at each factor, the proportion of variance explained by Factor 1 was $69.31 \%$ while Factor 2 explained $18.83 \%$ and Factor 3 explained $14.51 \%$.

\section{Table 6}

Factor Loadings Representing a Three-Factor Solution

\begin{tabular}{|c|c|c|c|c|}
\hline Item & Item Description & Factor 1 & Factor 2 & Factor 3 \\
\hline ESSY2 & I set my own goals. & .50 & & \\
\hline ESSY3 & I have achieved goals. & .50 & & \\
\hline ESSY3 & I set goals with my group/team. & .64 & & \\
\hline ESSY5 & I have worked hard to achieve a goal. & .51 & & \\
\hline ESSY6 & I set long term goals. & .68 & & \\
\hline ESSY7 & I set short term goals. & .69 & & \\
\hline ESSY8 & $\begin{array}{l}\text { I have specific plans/steps to achieve my } \\
\text { goals. }\end{array}$ & .77 & & \\
\hline ESSY9 & I have told others about my goals. & .64 & & \\
\hline ESSY10 & I regularly reviewed my goals. & .74 & & \\
\hline ESSY11 & $\begin{array}{l}\text { I have faced challenges (e.g., cut from a } \\
\text { team, suffered an injury, had to sit out } \\
\text { games, etc.) }\end{array}$ & & & .52 \\
\hline ESSY 12 & I have lost an important game/competition. & & & .66 \\
\hline ESSY13 & I have struggled to learn a new skill. & & & .64 \\
\hline ESSY14 & $\begin{array}{l}\text { I have played on a team where I have not } \\
\text { gotten along with some people. }\end{array}$ & & & .47 \\
\hline ESSY17 & $\begin{array}{l}\text { I have made a mistake that cost me or my } \\
\text { team. }\end{array}$ & & & .50 \\
\hline ESSY20 & $\begin{array}{l}\text { I have had the opportunity to develop new } \\
\text { skills. }\end{array}$ & & .78 & \\
\hline ESSY21 & $\begin{array}{l}\text { I have worked hard to develop a specific } \\
\text { skill. }\end{array}$ & & .80 & \\
\hline ESSY22 & $\begin{array}{l}\text { I have had the opportunity to develop a } \\
\text { wide range of physical skills. }\end{array}$ & & .72 & \\
\hline ESSY23 & $\begin{array}{l}\text { I had a lot of help from others to improve } \\
\text { skills. }\end{array}$ & & .59 & \\
\hline ESSY24 & I have worked on skills on my own time. & & .45 & \\
\hline
\end{tabular}


Despite the adequate factor loadings and eigenvalues above 1.00 , it is important to note that communality scores for some items were below the suggested cutoff value. Communality scores are presented in Table 7 and show a few items below .40. These values are noteworthy, but, ultimately, the items were retained because they loaded above .32 and are consistent with empirical evidence and experiences reported by interview participants.

Table 7

Communalities for ESSY Items

\begin{tabular}{llc}
\hline Item & Item Description & Communality \\
\hline ESSY2 & I set my own goals. & .48 \\
ESSY3 & I have achieved goals. & .39 \\
ESSY4 & I set goals with my group/team. & .42 \\
ESSY5 & I have worked hard to achieve a goal. & .50 \\
ESSY6 & I set long term goals. & .51 \\
ESSY7 & I set short term goals. & .52 \\
ESSY8 & I have specific plans/steps to achieve my goals. & .56 \\
ESSY9 & I have told others about my goals. & .35 \\
ESSY10 & I regularly reviewed my goals. & .57 \\
ESSY11 & I have faced challenges (e.g., cut from a team, & .36 \\
& suffered an injury, had to sit out games, etc.) & .46 \\
ESSY12 & I have lost an important game/competition. & .44 \\
ESSY13 & I have struggled to learn a new skill. & .22 \\
ESSY14 & I have played on a team where I have not gotten & .25 \\
& along with some people. & .58 \\
ESSY17 & I have made a mistake that cost me or my team. \\
ESSY20 & I have had the opportunity to develop new skills. & .64 \\
ESSY21 & I have worked hard to develop a specific skill. & .53 \\
ESSY22 & I have had the opportunity to develop a wide & \\
ESSY23 & range of physical skills. & .43 \\
ESSY24 & I have worked on skills on my own time. & .29 \\
\hline
\end{tabular}

The comparison of eigenvalues to the outcome of the Monte Carlo Parallel Analysis showed the three-factor solution with an eigenvalue of 1.20 was below the critical value of 1.30 estimated by the parallel analysis (Franklin et al., 1995; Watkins, 
2005). However, Lim and Jahn (2019) suggest that, due to some of the limitations with parallel analysis, values within $+/-1$ of the critical value can be considered for retention. As such, the 19-item, three-factor solution was retained.

\section{Discussion}

The purpose of Study Two was to explore the factor structure of the proposed ESSY. The first iteration contained 27 items and was expected to load onto three factors. Results from the first exploratory factor analysis did not support a three-factor model and items were systematically removed for each subsequent iteration. A final 19-item, three factor model was retained and provides a measure of experience in sport as assessed by goal setting, skill development, and adversity.

Several items were removed because they did not load onto a factor or crossloaded onto multiple factors. In addition, ESSY1 was removed based on consideration of the distribution of scores and how meaningful it was to the overall goal setting construct. The greatest number of items were retained for the goal setting factor and this factor explained the largest proportion of variance. Five factors were retained for skill development and adversity, which is above the three-item minimum typically recommended for factor analysis (Kelloway, 2014). It was felt that the remaining items provided a concise measure of experiences in sport and accurately covered the key components of each construct.

Communalities were low for some of the items in the final EFA. The mean communality score for skill development was $h^{2}=49.4$, which is above the recommended cut-off value of .40 , suggesting that there is sufficient shared variance being caused by the underlying factor. The average communality for adversity was $h^{2}=$ 
34.6. This is close to the cut-off value but may be indicative of items that do not share much variance (Watkins, 2018). Yet, taking into consideration the additional criteria for item/factor retention and that items loaded satisfactorily onto their respective factors, it was decided that retaining the adversity subscale for the confirmatory factor analysis in Study Three was warranted.

The items retained are consistent with literature and/or with experiences reported by interview participants. Item reduction was anticipated as part of the exploratory factor analyses in the scale development process as development followed recommendations that items be purposefully overinclusive (Simms, 2008). Importantly, the items grouped together within their expected factor to provide meaningful representations of the threefactor model of experiences in sport for youth. Thus, the 19-item, three-factor ESSY was maintained for Study Three.

\section{Study Three: Testing the Sport-Based Model of PYD}

The purpose of Study Three was to confirm the factor structure of the ESSY reported in Study Two and to test the proposed Sport Based Model of Positive Youth Development presented in Figure 3. Based on results from Study Two, it was expected that the factor structure of the ESSY would be a good fit to the data and that paths from the latent constructs (goal setting, adversity, and skill development) to each item would be significant. Furthermore, based on the literature reviewed and findings from Study One, it was expected that the proposed sport-based model of positive youth development would be a good fit to the data. Specifically, it was expected that the model would explain a significant proportion of the variance in PYD outcomes of competence, confidence, character, caring, and connection. Finally, it was hypothesized that sport 
climate variables would interact with experiences in sport variables to strengthen the association between experiences in sport and PYD outcomes.

\section{Figure 3}

Proposed Sport-Based Model of Positive Youth Development to be Tested

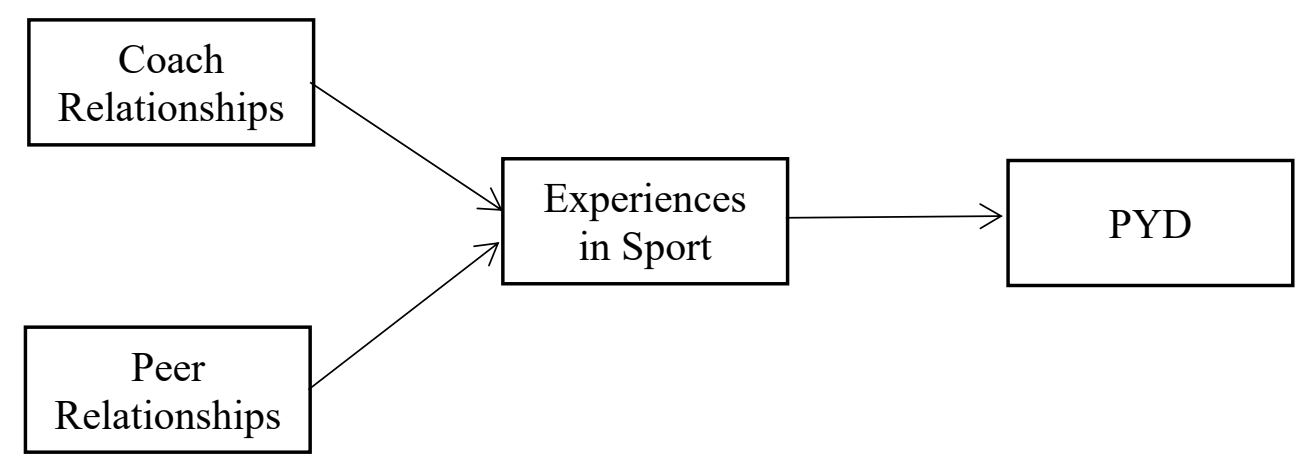

\section{Method}

\section{Participants}

Participants were 453 undergraduate students between the ages of 17 and 20 ( $M_{\text {age }}$ $=18.46 ; 66 \%$ identified as female) who had participated in an organized sport within the 12-months prior to survey completion. Participants were enrolled in introductory psychology courses and received course credit for their participation.

Half of participants reported that their mother was a college or university graduate (50.9\%) while $14.7 \%$ had graduated high school, $15.9 \%$ had some college/university, and $14.2 \%$ had a graduate degree (remaining percent was divided among some high school and some graduate). Almost half the sample reported living in a suburb (47.6\%) and had played organized sport for an average of 9.61 years $(s d=4.47)$. Soccer was played by $62.4 \%$ of the sample, followed by swimming (36.6\%), basketball $(32.4 \%)$, dance (32.4\%), and hockey (30.5\%) as the top five sports listed. 
There was substantial variability in the years involved in sport and this extended to years in recreational sport $(M=5.49, s d=4.28)$ and years spent in competitive sport $(M=5.11, s d=4.27)$. Just over half of the participants were currently playing at the competitive level (53.5\%). Roughly $60 \%$ of the participants reported engaging in other extra-curricular activities (i.e., music, art, volunteering, youth group, church group). Volunteering was the most reported activity (34.5\%) followed by music (13.9\%), art (5.7\%), church group (4.7\%) and youth group (1.2\%).

\section{Procedure}

Participants were recruited using a secure online network. Students in introductory psychology courses are given the option to participate in research studies in exchange for course credit and the study was advertised on this secure network (see Appendix G). If students met the criteria (i.e., between the ages of 17 and 20, played an organized sport in the previous 12 months), they could self-select to participate in the study. The questionnaire was accessed and completed online using personal devices (e.g., laptop, cell phone, tablet). All participants were required to provide consent before accessing the study (see Appendix $\mathrm{H}$ for participant consent form) and were provided with a debriefing form with additional information about the study and contact information should they require support after completing the study (see Appendix $\mathrm{K}$ for debrief).

\section{Measures}

Participants completed a series of questionnaires as well as providing basic demographic information including age, gender, mother's education level, amount of 
time playing sport, type of sports played, etc. The full background questionnaire can be seen in Appendix L.

Teammate Relationships. To assess teammate relationships, participants completed the Peer Motivational Climate in Youth Sport Questionnaire (PeerMCYSQ; Ntoumanis \& Vazou, 2005; see Appendix N). The PeerMCYSQ is a 21-item questionnaire that assesses motivational factors related to improvement, relatedness support, effort, intrateam competition and support, and intrateam conflict. Participants were asked to rate their level of agreement with each of the statements using a 7-point scale from strongly agree (1) to strongly disagree (7). The Peer MCYSQ has been widely used with adolescent populations and has shown strong internal consistency (Jõesaar et al., 2012; van de Pol et al., 2012). The subscales of improvement ( $\alpha=.82)$, relatedness $(\alpha=.71)$, effort $(\alpha=.87)$, and intrateam conflict $(\alpha=.78)$ appeared to be reliable for the present study. However, intrateam competition had a very low value for reliability $(\alpha=.33)$ suggesting the items were not reliable or consistent measures of the construct.

Coach Relationship. To obtain a measure of coach relationship, the Motivational Climate Scale for Youth Sports was completed (MCSYS; Smith et al., 2008; see Appendix O). The MCSYS is a 12-item questionnaire designed to measure ego and mastery coach created sport climate. Participants were asked to rate how true each item was based on their experience with their coach and rate on a 5-point scale from not at all true (1) to very true (5). The MCSYS is a validated measure and has shown good internal consistency in previous research (O’Rourke et al., 2014; Smith et al., 2009). For the present study, both master $(\alpha=.82)$ and ego $(\alpha=.76)$ showed satisfactory internal consistency. 
Experiences in Sport. Experiences in Sport Scale for Youth (ESSY) was used to provide a measure of experiences in sport. The ESSY is a 19-item questionnaire that was designed to assess experiences in sport related to goal setting, adversity, and skill development. Participants are asked to rate how frequently they experience each item using a 5-point scale Never (1) to Always (5). Reliability appeared to be good for goal setting $(\alpha=.87)$ and skill development ( $\alpha=.81)$ but was not strong for adversity $(\alpha=.67)$.

Positive Youth Development. The 5Cs of Positive Youth Development - Short Form for Older Adolescents (Geldhof et al., 2014; see Appendix P). This is 34-item measure that assesses participants' perceived levels of competence, confidence, connections, character, and caring. The questionnaire asked participants to respond to items using 5-point scales. Depending on the group of items, participants were asked to rate their level of agreement, how important an item was to them, or how true a statement was of them. The first set of items (items 1-12) presented participants with two statements, then asked participants to select the statement that best reflected them and their experiences, and then to rate how true the statement was of them. The reliability of the subscales varied with most showing less than satisfactory values for alpha. Caring was the most reliable $(\alpha=.83)$ followed by confidence $(\alpha=.77)$, connection $(\alpha=.67)$, character $(\alpha=.64)$, and competence $(\alpha=.56)$.

The PYD-SF has been used and validated with late-adolescent samples (Geldhof et al., 2014). The authors suggest using a bifactor model when incorporating the PYD-SF into a structural equation model. The present study defined PYD in this way with competence, confidence, connections, character, and caring loading onto a higher order PYD construct in the structural equation models. Note that, following school board 
guidelines, two questions about connections with racialized members of the community were removed from the questionnaire (questions asked participant to indicate how others would rate them on knowing a lot about people of other races and enjoying being with people who are of a different race than I am).

\section{Results}

The analyses for Study Three were carried out in sequence. This began with a CFA to confirm the factor structure of the ESSY, followed by testing a measurement model with all observed and latent variables, and then an SEM with paths between latent variables defined to test the Sport-Based Model of Positive Youth Development. Prior to any of these steps, the dataset was cleaned, and assumptions tested. Both SPSS and MPlus 8 programs were used to perform the analyses.

\section{Cleaning the Data and Testing Assumptions}

Missing data was assessed using a missing value analysis and a visual inspection of the data. No variable had more than $10 \%$ missing values and $83.89 \%$ of cases had complete data. Additionally, no single item had more than 5\% missing data. Of the 453 participants, 81 were missing data on at least one item with 12 participants having between 13 and 103 points of data missing. 27 participants were missing between two and ten points of data while 42 participants had only one missing value. Participants with missing data points were compared to participants without missing data and no significant patterns were detected. Maximum likelihood was used to estimate missing values for all analyses.

The dataset was evaluated to ensure that it met the requirements to perform a confirmatory factor analysis (CFA) and structural equation modeling (SEM). Normality, 
linearity, collinearity, and the presence of univariate and multivariate outliers were assessed using visual inspection of histograms, scatterplots, boxplots, as well as correlation coefficients, skewness and kurtosis values, and Mahalanbois Distance (MD) scores. Probability was calculated for MD scores and a visual inspection of data showed that patterns of careless responding were present for 30 participants. Scores were not consistent with what would be expected in the population and indicated respondents were not reading the item (e.g., answering strongly agree for all items in the scale). As a check, scores from the 30 participants were compared to scores from the remaining participants and showed a significant difference between the two groups. These participants were removed from the dataset and analyses continued with 423 participants. All other assumptions were met, and no other changes were made to the dataset.

\section{General Plan of Analysis}

As mentioned, analyses for Study Three were carried out in two stages. The first was to assess the factor structure of the ESSY proposed based on Study Two findings. The second stage was to test the structural equation model by assessing the association between relationships in sport, sport experiences, and positive youth development outcomes.

A CFA falls under the umbrella of structural equation modeling as it allows for the testing of complex associations (i.e., multiple independent and dependent variables; Geiser, 2013). Unlike an SEM, a CFA does not test linear/directional associations between latent variables but rather allows the latent variables to covary (Geiser, 2013). An SEM allows for complex linear associations between variables to be tested by comparing the covariance matrix proposed by the model and the observed covariance 
matrix. The more similar the patterns of the covariance matrices, the better the "fit" of the model (Kelloway, 2014). SEM typically involves a two-step process where a measurement model is tested first followed by the proposed structural model.

Prior to running the analyses, each model (i.e., CFA, measurement model, SEM) was assessed to ensure it was identified. In order to provide an accurate solution and be able to estimate the model's free parameters, a model should be just-identified or overidentified (Kline, 2016). The model had more observations than parameters (Kline, 2016) and a path coefficient for each of the latent variables was fixed to 1.0 (Wang \& Wang, 2012) to ensure that the model was overidentified. Each model was estimated using Maximum Likelihood and estimation was evaluated using several indicators of model fit (e.g., incremental fit indicators, absolute fit indicators). Specifically, the models were assessed to see if they met the following criteria: a non-significant chi-square, a comparative fit index (CFI) and Tucker-Lewis Index (TLI) greater than .90, a Root Mean Square Error of Approximation (RMSEA) less than .06, and a Standardized Root Mean Square Residual (SRMR) less than .08 (Wang \& Wang, 2012). RMSEA also provides confidence intervals and probability associated with the close-fit test (i.e., that RMSEA is less than .05). In addition, $\mathrm{R}^{2}$ values and path loadings were used to further assess model fit.

\section{The Confirmatory Factor Analysis}

The CFA tested the proposed multidimensional model presented in Figure 4. As can be seen in Figure 4, the three correlated latent variables (goal setting, adversity, skill development) are predicting their corresponding indicators (i.e., observed variables). An 
error term for each observed variable was also included to account for variance not attributable to the latent factors. The error terms are independent and do not covary.

Figure 4

Proposed Factor Model of the ESSY

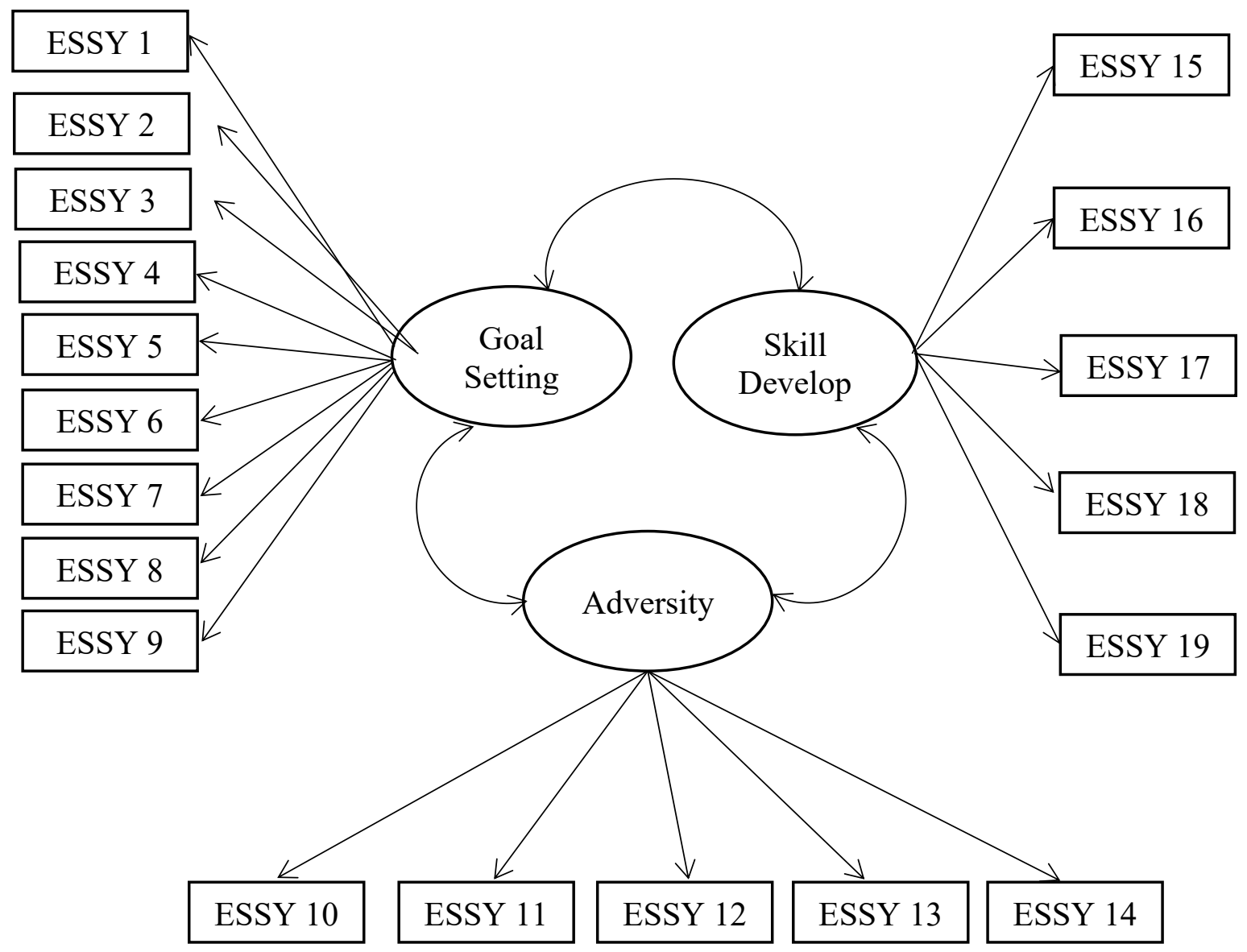

As the purpose of these analyses was to assess the factor structure of a newly developed scale, competing models were also tested. The first competing model was a second-order factor model where goal setting, adversity, and skill development loaded onto a second factor of experiences in sport. The second competing model tested a single factor model where each observed variable was caused by a single latent variable (i.e., experiences in sport). Finally, a model where the latent variables were treated as 
orthogonal was tested. To evaluate the relation with specific sociodemographic factors, covariates were assessed using a MIMIC model (Wang \& Wang, 2012). Significant covariate pathways can show differences between groups on latent variables. This can be problematic if differences are a result of measurement invariance as opposed to group differences.

\section{Results of Confirmatory Factor Analysis}

The results of the CFA showed the proposed three-factor model to be a satisfactory fit to the data. The chi-square statistic was significant $\left(\chi^{2}=343.77, d f=149\right.$, $\mathrm{p}<.001)$ but, due to the way it is calculated, the statistic is sensitive to sample size and can increase Type 1 error (McNeish, 2020). Looking at the other indicators of fit, all are equal to or above the specified cutoff values; $\mathrm{CFI}=.93, \mathrm{TLI}=.92, \mathrm{RMSEA}=.06,(90 \%$ CI $[.05, .06] ; \mathrm{p}=.106)$, and SRMR $=.05$. The standardized factor loadings for the paths from the observed variables to their respective latent variables are presented in Table 8 .

As can be seen, each of the observed items loads significantly and all are above the critical value of .40 (Wang \& Wang, 2012). The covariance between Skill Development and Goal Setting was significant (.66) as was the covariance between Skill Development and Adversity (.24). However, Adversity and Goal Setting were not significantly associated.

Competing models were then assessed. The higher order model with Goal Setting, Adversity, and Skill Development loading on an overarching latent variable (i.e., Experiences) had fit indices that suggested a good fit. However, a Heywood case was detected in the data (a negative residual variance, Geiser, 2013; Kline, 2016). 


\section{Table 8}

Standardized Factor Loadings of the ESSY Items

\begin{tabular}{llrc}
\hline Latent Variable & Observed Variable & Estimate & Standard Error \\
\hline Goal Setting & ESSY1 & .73 & .03 \\
& ESSY2 & .67 & .03 \\
& ESSY3 & .58 & .04 \\
ESSY4 & .75 & .02 \\
& ESSY5 & .69 & .03 \\
& ESSY6 & .64 & .03 \\
& ESSY7 & .70 & .03 \\
ESSY8 & .51 & .04 \\
Adversity & ESSY9 & .70 & .03 \\
& ESSY10 & .58 & .04 \\
& ESSY11 & .62 & .04 \\
& ESSY12 & .62 & .04 \\
& ESSY13 & .47 & .05 \\
& ESSY14 & .54 & .05 \\
ESkill Development & .75 & .03 \\
& ESSY16 & .75 & .03 \\
& ESSY17 & .77 & .03 \\
& ESSY18 & .63 & .03 \\
& ESSY19 & .58 & .04 \\
\hline
\end{tabular}

Note. All estimates are significant at $\mathrm{p}<.001$

After evaluating the model and data, it was determined that the impossible value was an indication that the higher-order model was not an adequate solution for the data and not due to misspecification or issues with the data (Kline, 2016). The fit indices for the other two models are presented in Table 9 and show that neither model provides an appropriate solution.

A multiple indicator multiple cause model (MIMIC) was run to assess demographic variables as covariates in the CFA model. The variables added to the model were age, gender, socio-economic status, level of current play (recreational versus competitive), years playing organized sport at the competitive level, years playing organized sports, and environment (urban, rural, suburban). Socio-economic status was 
found to be a significant covariate with Goal Setting $(-.46, \mathrm{p}<.05)$ and Skill Development $(.55, \mathrm{p}<.05)$. In addition, Skill was found to covary with level of play $(.32, \mathrm{p}<.001)$ and age $(-.35, \mathrm{p}<.01)$. No other paths were significant.

\section{Table 9}

Fit Indices for Competing Models

\begin{tabular}{lcc}
\hline Fit Indices & Single Factor Model & Orthogonal Factor Model \\
\hline Chi-Square & 938.72, & 517.63 \\
& $d f=152$ & $d f=152$ \\
CFI & .71 & .80 \\
TLI & .67 & .86 \\
RMSEA & .11 & .85 \\
SRMR & .09 & .15 \\
\hline
\end{tabular}

The findings of the CFA support the factor structure of the ESSY. The model was found to be a good fit to the data based on fit indices and factor loadings. Potential competing models were shown to not be a good fit to the data, further supporting the ESSY model. The presence of significant covariates indicated the need to assess demographic variables as influential factors in the sport-based model of positive youth development to be tested as these may differentially influence experiences in sport. No modifications were made to the ESSY and analyses proceeded with the 19-item, threefactor model.

\section{The Structural Equation Model}

With the 19-item, three-factor structure of the ESSY confirmed, the proposed sport-based model of PYD was tested. The full model that was tested is presented in Figure 5 and shows the proposed relations between the PYD climate variables (coach and peer relationship), experiences in sport variables, and the PYD outcomes variables. 


\section{Figure 5}

Structural Equation Model

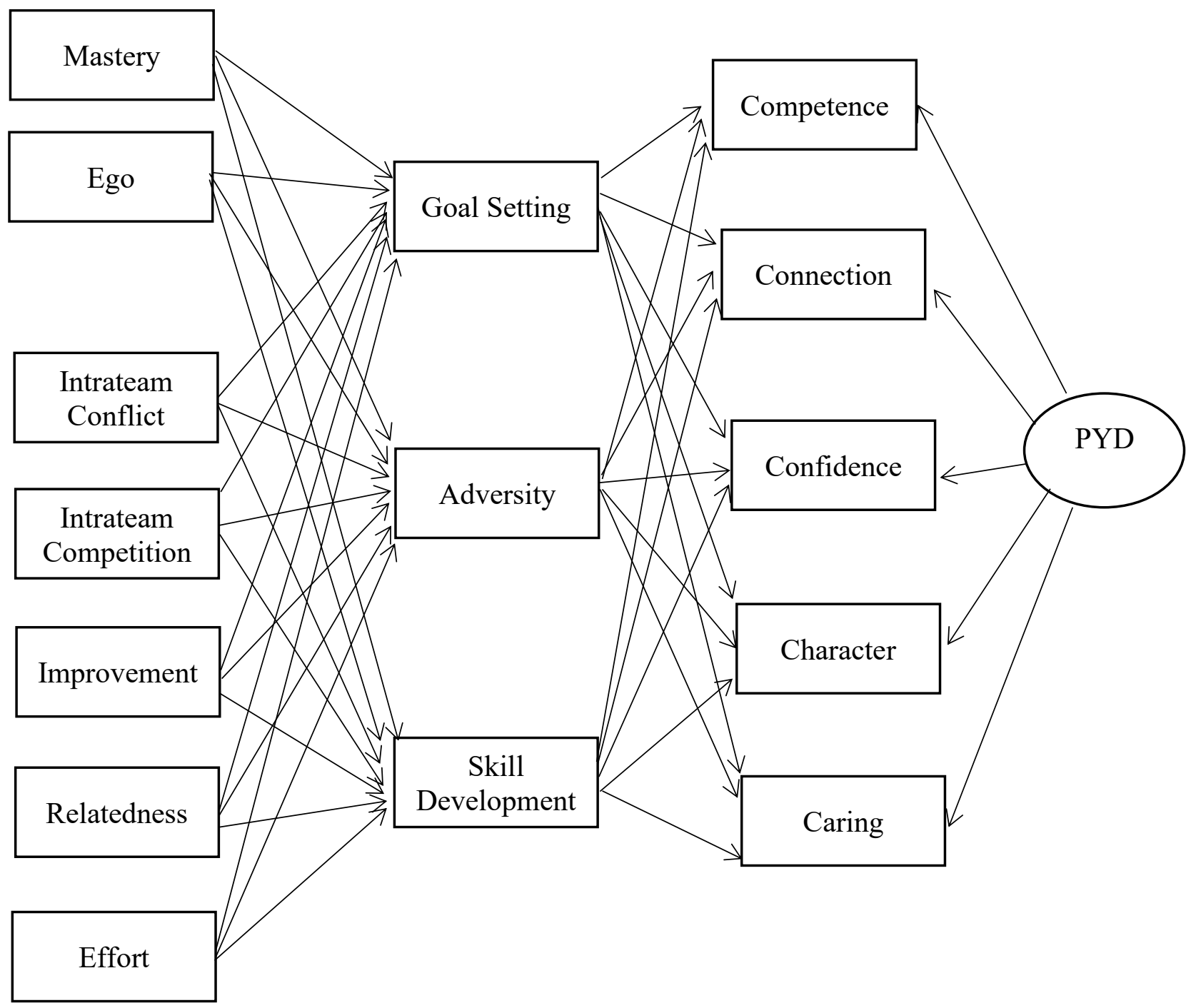

\section{Descriptive Statistics}

Prior to running inferential statistics, descriptive statistics were obtained. Means and standard deviations for the key study variables are presented in Table 10. As can be seen, participants reported high levels of a mastery coach climate and a lower level of an ego coach climate. Similarly, higher scores related to positive peer climates were reported (improvement, relatedness, and effort) and lower levels of negative peer climate 
(intrateam conflict and competition). Participants reported a high level of caring but did not necessarily perceive themselves to be competent, as assessed by the PYD-SF.

\section{Table 10}

Means and Standard Deviations of Study Variables

\begin{tabular}{lcc}
\hline Variable Name & Mean & Variance \\
\hline Goal Setting & 3.76 & .43 \\
Adversity & 3.04 & .38 \\
Skill Development & 3.96 & .38 \\
Coach - Mastery & 4.02 & .51 \\
Coach - Ego & 2.64 & .70 \\
Peer - Intrateam conflict & 4.00 & 2.18 \\
Peer - Intrateam competition & 4.68 & .69 \\
Peer - Improvement & 5.63 & 1.06 \\
Peer - Relatedness & 5.59 & 1.12 \\
Peer - Effort & 5.89 & .97 \\
Competence & 2.74 & .38 \\
Confidence & 3.10 & .51 \\
Character & 3.61 & .30 \\
Caring & 4.09 & .44 \\
Connection & 3.14 & .35 \\
\hline
\end{tabular}

The correlation table is presented in Table 11 and shows that many of the study variables were significantly correlated. Goal setting and skill development were significantly correlated with almost all study variables, including the PYD outcomes. Interestingly, and in contrast with expectations, adversity was not significantly correlated with any of the PYD outcome variables. Some variables were unexpectedly not significantly related, such as a mastery coach climate and perceived competence and mastery coach climate and confidence. 


\section{Table 11}

Correlations of Key Study Variables

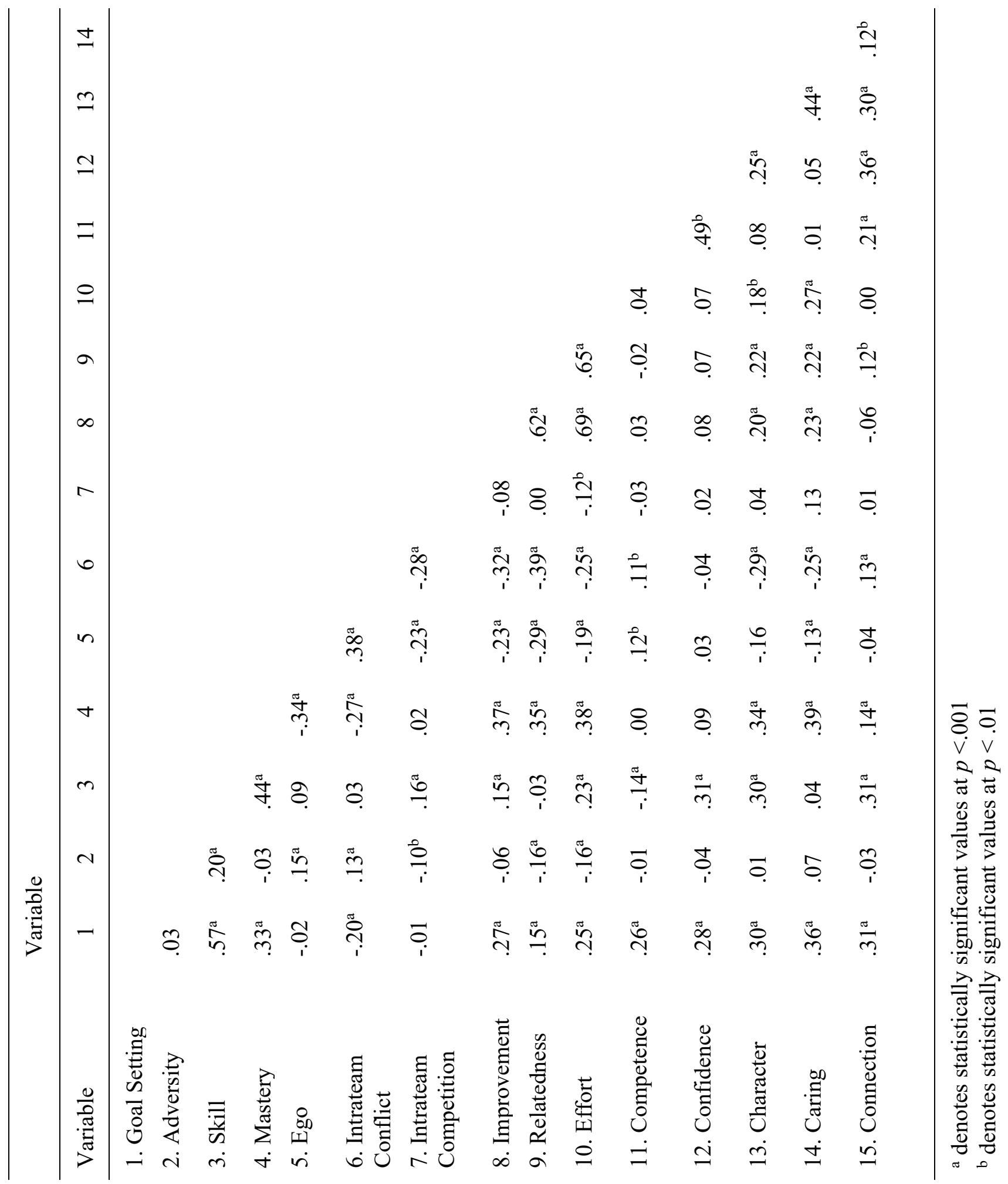


Differences between the key study variables by socio-demographic variables were tested using multiple analysis of variance (MANOVA). Experiences in sport significantly differed by level of play $(F(3,329)=8.24, p<.001$; Wilk's $\lambda=.93)$ with participants playing competitive sport reporting higher levels of goal setting $(F=(1,331)=16.21, p$ $<.001)$ and skill development $(F=21.62(1,331), p<.001)$. Scores also differed significantly according to gender for the 5 Cs of PYD $(F(5,405)=10.90, p<.001$; Wilk's $\lambda=.88)$, coach climate $(F(2,417)=4.68, p<.05 ;$ Wilk's $\lambda=.98)$, and peer climate $(F(5$, $414)=9.55, p<.001 ;$ Wilk's $\lambda=.90)$. Specifically, female participants reported significantly higher caring scores $(F(5,405)=15.25, p<.001)$, intrateam competition scores $(F(1,418=19.52, p<.001)$,$) , and relatedness scores (F(1,418)=5.28, p<.05)$, $)$. Conversely, male participants reported higher competence $(F(5,405)=33.00, p<.001)$, confidence $(F(5,405)=12.5, p<.001)$, $)$, ego $(F(1,418)=9.32, p<.01)$, $)$, and intrateam conflict scores $(F(1,414)=36.56, p<.001)$. Scores did not differ significantly based on socio-economic status or environmental setting (i.e., urban, rural, suburban).

\section{Sport-Based Model of PYD}

The proposed model involved latent variables so the measurement model was first assessed. Similar to a CFA, the measurement model assesses the association between indicator items (i.e., observed variables) and their associated latent variable. Only the paths from the indicator items to their latent variable are defined. Latent variables were free to covary and each variable (both manifest and latent) had an associated residual term. A path coefficient for each latent variable was fixed to ensure that the model could be identified (Wang \& Wang, 2012). The full measurement model was tested but failed to 
converge. It was then decided that coach and peer models would be tested separately to reduce the number of parameters being estimated by the model.

\section{Coach Relationship Model}

The indicator items for coach relationship (ego and mastery latent variables), experience in sports (goals, adversity, skill development latent variables), and the positive youth development outcomes (confidence, competence, character, connection, and caring latent variables) were defined for the measurement model. The model converged and terminated normally. The fit indices suggested the model was a mediocre fit to the data. The chi-square was significant $\left(\chi^{2}=3682.97, d f=1724, \mathrm{p}<.001\right)$ and values for incremental fit indices TLI and CFA were below the critical value $(\mathrm{CFI}=.79$, TLI $=.77)$. However, RMSEA and SRMR values were acceptable (RMSEA = .05, (90\% CI [.048, 053], $\mathrm{p}=.316) ; \mathrm{SRMR}=.06)$. Path coefficients were significant for each of the defined paths with standardized factor loadings ranging from .20 to .81. Two PYD-SF items failed to load significantly onto the Connection factor. Analyses were run without the two items but no improvements to model fit were evident. As the items did not load significantly on their respective variable and had no discernable impact on the model, they were removed for the structural equation model.

Although the incremental fit indices were not satisfactory, because RMSEA and SRMR were satisfactory and each of the path coefficients were significant, it was decided that running an SEM was justified. Not unexpectedly, the model was not a good fit to the data. The chi-square was significant $\left(\chi^{2}=395.65, d f=20, \mathrm{p}<.001\right)$, incremental fit indices were well below the cut-off values $(\mathrm{TLI}=0.00 ; \mathrm{CFI}=.49)$ and both $\mathrm{RMSEA}$ and SRMR were above $.06(\mathrm{RMSEA}=.21,90 \% \mathrm{CI}[.19-.22], \mathrm{p}<.001 ; \mathrm{SRMR}=.16)$. 
Covariates of gender, SES, age, and level of play were tested in the model. None of the possible covariates significantly improved the model nor did they significantly change the path coefficients. Modification indices were also reviewed but none of the suggestions provided significant improvement to model fit or they did not represent a conceptually meaningful association.

\section{Peer Relationship Model}

Indicator items for peer relationships (intrateam competition/ability, intrateam conflict, improvement, relatedness support, effort latent variables), experience in sport (goals, adversity, skill development latent variables) and PYD (confidence, competence, character, connection, and caring latent variables) were defined for the measurement model. Similar to the coach relationship model, the peer relationship model converged, terminated normally, and was a mediocre fit to the data. The chi-square was, again, significant $\left(\chi^{2}=5026.21, d f=2406, \mathrm{p}<.001\right)$, incremental fit indices were below the critical value $(\mathrm{CFI}=.78, \mathrm{TLI}=.77)$ and $\mathrm{RMSEA}$ and SRMR values were acceptable $(\mathrm{RMSEA}=.05,(90 \%$ CI $[.048,052], \mathrm{p}=.620) ; \mathrm{SRMR}=.06)$. All path coefficients loaded significantly onto their respective latent variable.

Paths between latent variables were defined and the structural equation model executed. Consistent with the coach model, the defined model was not a good fit to the data. The chi-square was, again, significant $\left(\chi^{2}=394.17, d f=35, p<.001\right)$, CFI were both well below the .90 cut-off $(\mathrm{CFI}=.49, \mathrm{TLI}=.02)$ and RMSEA and SRMR values were above $.06(\mathrm{RMSEA}=.15,90 \%$ CI $[.14, .17], \mathrm{p}<.001 ; \mathrm{SRMR}=.14)$. This finding was contrary to expectations that peer relationship variables and experiences in sport variables would explain a significant proportion of the PYD outcomes. Covariates of 
gender, SES, age and level of play were tested in the model but did not significantly improve the model nor did they significantly change path coefficients. In addition, modification indices were reviewed but none represented a significant contribution to model fit or were not conceptually meaningful.

\section{Testing Interactions}

Based on findings from the qualitative study of experiences in sport, it was hypothesized that coach and peer relationships would impact the association between experiences in sport and PYD outcomes. It was expected that a more positive coach and peer climate would strengthen the association between goal setting, adversity, and skill development in sport and PYD outcomes. To test this hypothesis, an interaction between each of the coach and peer climate variables and the experience in sport variables was also tested (e.g., mastery x goals, mastery x skills, ego x goals) to determine if coach/peer climate moderated the association between experience variables and PYD outcomes. Contrary to expectations but consistent with the overall model fit, none of the interactions were significant.

\section{Experience in Sport and PYD Outcomes}

As the overarching purpose of this study was to understand the association between experiences in sport and positive youth development, a simplified model to test this specific relation was run. Experience in sport variables were tested as predictors of the bifactor PYD model. The model provided a better fit than the previously tested models. A modification was made to add a covariance path between character and caring. Research with the $5 \mathrm{C}$ model of PYD has pointed to the similarities between character and caring in how they are measured (e.g., Conway et al., 2015). The finding that caring 
was not a significant indicator of PYD but significantly associated with character is consistent with that criticism.

For the final model, the chi-square was significant $\left(\chi^{2}=19.86, d f=4, p<.001\right)$, CFI (.96) was satisfactory while TLI (.82) fell below the recommended critical value, RMSEA was not significant but above the recommended value $(\mathrm{RMSEA}=.09,90 \% \mathrm{CI}$ $[.06, .14], \mathrm{p}=.03)$, and SRMR (.04) was satisfactory. As with each of the other models, covariates (i.e., gender, level of play, SES) were tested in the model but did not significantly alter the fit of the model or the path coefficients. The significant, standardized factor loadings are presented in Figure 6.

\section{Figure 6}

Standardized Factor Loadings for the Simplified Model

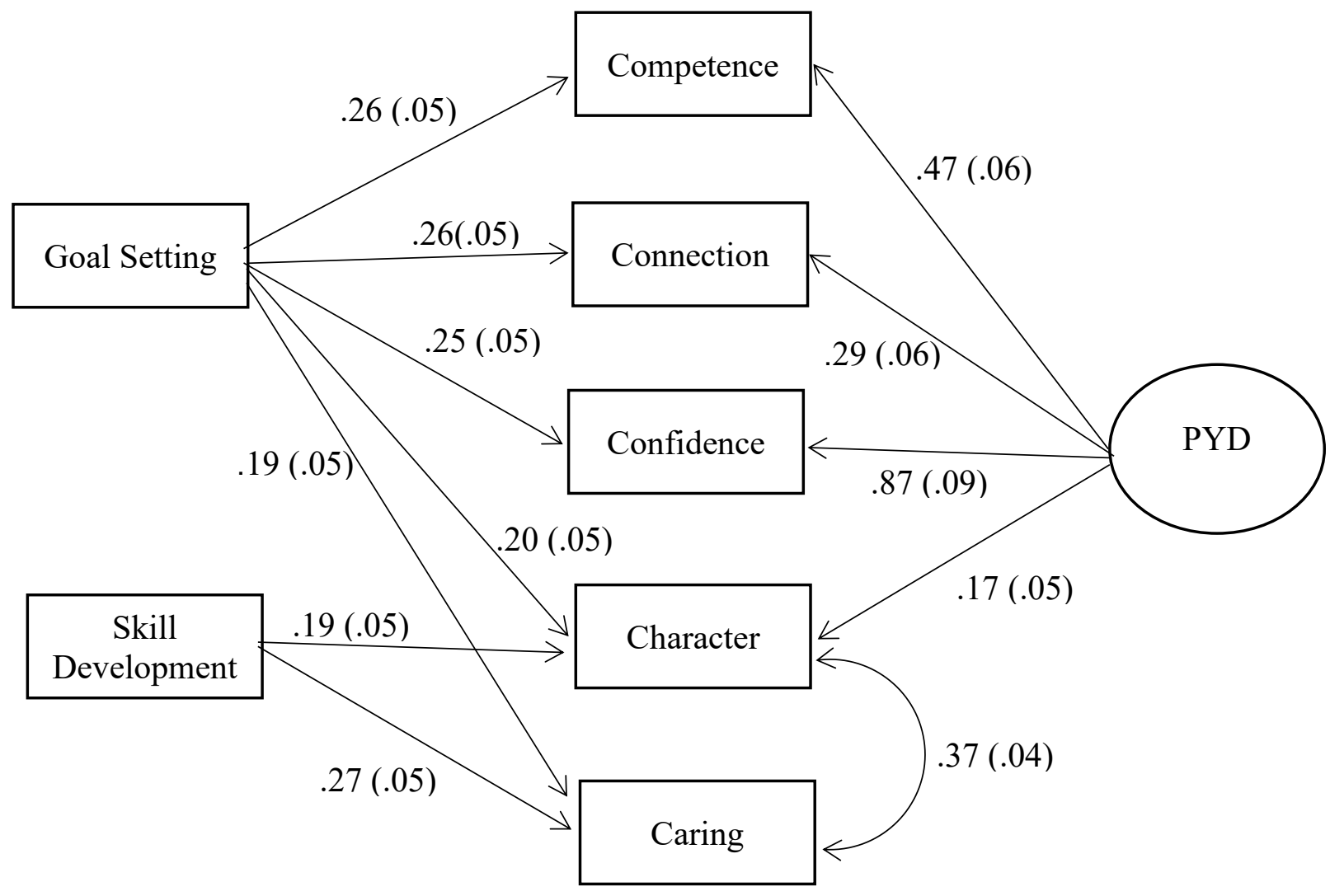


As can be seen in Figure 6, goal setting significantly predicts each of the five PYD outcomes, with path coefficients ranging between .19 and .26. Skill development was significantly associated with character and caring. Unexpectedly, adversity was not significantly associated with any of the PYD outcomes. Overall, the model explained $29.1 \%$ of the variance in competence, $82.8 \%$ of the variance in confidence scores, $14.8 \%$ of the variance in character scores, $17.2 \%$ of caring, and $15.4 \%$ of connection scores.

\section{Testing Alternative Models}

To be scientifically rigorous, it is often recommended that alternative models be tested in structural equation modeling (e.g., Wei \& Lu, 2007; Weston \& Gore, 2006). This ensures that the accepted model most accurately represents the data (Weston \& Gore, 2006). As such, multiple alternative models were tested in Study Three. One model involved parceling of indicator items for each of the latent factors, which has been shown to improve model fit (Matsunaga, 2008). In addition, models involving positive elements of coach/peer climate (i.e., improvement, relatedness, effort, and mastery latent variables) and models without the experience variables (i.e., coach climate predicting 5 Cs of PYD and peer relationship climate predicting 5 Cs of PYD) were tested. None of these alternative models were a satisfactory fit to the data nor did they represent an improvement in model fit.

\section{Discussion}

The purpose of Study Three was twofold. The first objective was to confirm the factor structure of the Experience in Sport Scale for Youth (ESSY) and the second objective was to test the proposed Sport-based Model of PYD (see Figure 4). The factor structure of the ESSY was confirmed but hypotheses were only partially supported for 
testing the sport-based model of PYD. Confirmation of the ESSY factor structure indicates that the items and three-factor model represent a way to assess and categorize youth experiences in sport. Contrary to expectations, the proposed sport-based model of PYD did not fit the data. A simplified model with the experience in sport variables predicting the 5 Cs of PYD was tested and demonstrated significant links between some aspects of sports experience and some components of PYD. The following section discusses the findings.

\section{The Experience in Sport Scale for Youth}

Goal setting, skill development, and facing adversity were all hypothesized to be key experiences in organized sport. As such, an objective of Study Three was to assess the factor structure of the ESSY. Results of the CFA supported expectations that the proposed factor structure would be a good fit to the data. Each of the items loaded significantly onto their respective latent factors and indicators of model fit were satisfactory. In addition, competing models were tested and these supported the threefactor structure. Ultimately, findings provided support for the assertion that the items accurately represented the latent factors of goal setting, adversity, and skill development.

The impetus for this work was to understand what it is about playing organized sports that makes it a context for positive developmental outcomes. Studies have reported increased positive outcomes for those who participate in sports compared to other extracurricular activities (Larson, 2006). Furthermore, the key features of organized sport are consistent with what the developmental systems literature deems important for successful development, including a sustained adult-youth relationship, skill building opportunities (Lerner, 2014), working towards goals (Larson, 2000), and developing competence 
(Bronfenbrenner \& Morris, 1998). Although attempts have been made to assess experiences in sport, an adequate measure has not yet been developed. The ESSY may fill this gap.

The ESSY may provide an important way to assess some of the key experiential factors in the sport setting. As mentioned, a way to assess causal mechanisms within sport has been missing from the current knowledge base. Future work will be needed to further validate the ESSY and to better understand if goal setting, adversity, and skill development are associated with positive outcomes in various sport settings. This will include examining if these factors can be influenced by the PYD climate (i.e., parents, coaches, and peers) and if they transfer to life skills outside of the sport environment. Findings from Study Three suggests that both skill development and goal setting are valuable experiences for $5 \mathrm{C}$ outcomes. Incorporating other outcomes will be important to expanding the knowledge base as the $5 \mathrm{C}$ conceptualization of PYD has some criticisms, as previously discussed.

Facing adversity did not predict any of the 5 C PYD outcomes. This is one concept that has not received a great deal of attention outside of elite sport research. Elite athletes have described the value of facing and overcoming adversity and its relationship to characteristics such as resiliency (Brown et al., 2015; Galli \& Vealey, 2008). In the general PYD literature (i.e., not sport-based), resilience is consistently linked with PYD. It is viewed by some as the long-term outcome of PYD efforts and by others as an asset obtained through involvement in PYD contexts (see Lee et al., 2012 for review). Resilience science is an entire discipline that has leaned heavily on developmental systems theory to study how youth can overcome negative childhood experiences, such 
as abuse, maltreatment, and natural/humanitarian disasters (Lerner et al., 2019). This is obviously more challenging than the adversity that would be associated with losing a sporting competition.

In the development of the ESSY, it was suggested that experience with adversity in a psychologically safe environment, like a sporting event, would help prepare youth for dealing with more adverse situations in their life. Resiliency was not assessed in the present study as it is not part of the $5 \mathrm{C}$ conceptualization of PYD. It may be beneficial for future research to investigate the association between overcoming adversity in sport and resilience to understand if adversity represents an important developmental experience in the sport context.

A final point of discussion about adversity is consideration of the factor structure of the adversity subscale. In the EFA in Study Two, communalities were low for some of the items measuring adversity. One reason could be the need to include more items that would cover additional aspects of the adversity construct (Child, 2006). For example, adding items with ESSY19, I have made a mistake that cost me or my team, would incorporate different experiences around the idea of making mistakes in sport. Given the complexity of the adversity concept, future validation work with the ESSY should include incorporating additional items not previously considered into the adversity subscale.

\section{Sport-Based Model of PYD: Goal Setting, Skill Development and PYD}

Although results for the overall model were contrary to expectations, findings from the simplified model are consistent with developmental theory and, in particular, the importance of developing competence and confidence. Goal setting was strongly 
associated with all of the PYD outcomes, a finding that is supported by developmental and sport-based research. As previously discussed, goal setting is the impetus for several positive outcomes, both in sport and in youth development.

With regard to sport, research has shown goal setting to be an important part of sport performance and competitive outcomes. Goal setting is said to direct behaviour, focus effort, and increase motivation (Burton \& Weiss, 2008) while improving performance (e.g., Swain \& Hones, 1995; Weinberg et al., 2000). These are all very important to achieving success in athletic endeavors. Findings from this study support that goal setting in sport is also beneficial to development, particularly in the areas of competence and confidence.

Beginning with Bronfenbrenner and moving to developmental systems theory, competence has been consistently listed as an essential characteristic of development. Larson has identified the importance of pursuing and attaining goals for the development of competence and confidence in his conceptualization of PYD (Larson, 2011). Beyond competence and confidence, the findings from this study showed that goal setting was positively associated with each of the 5 Cs of PYD. This supports previous literature that show some of the specific psychosocial outcomes associated with goal setting. Danish et al., (2003) emphasized goal attainment as an essential piece of psychosocial development and Markstrom et al., (2005) argued that goal setting is instrumental in developing purpose and competence in Erikson's theory of identity development.

It was expected that skill development would be associated with each of the PYD outcome variables. However, given how skill development was conceptualized (e.g., I have worked hard to develop a specific skill), it does make sense that skill development 
was most strongly associated with character and caring. As stated in the definitions of the constructs in Table 4, skill development assessed the extent to which participants were self-directed and motivated in their skill development. As such, reporting high levels of skill development indicates, to some extent, that participants were more self-directed and motivated. It is also plausible that skill development requires individuals to become more self-aware and to work harder to develop skill through self-direction and motivation and it is this aspect of skill development that promotes character. Finally, skill-development could be argued to represent an achievement orientation that is task-focused and task orientation has been consistently linked with positive pro-social outcomes (Shields \& Bredemeier, 2008). Consequently, skill development, as assessed by the ESSY, is an important contributor to the development of character and caring.

\section{Development Over Time and Taking a Person-Centered Approach}

Findings from the present study did not provide a complete explanation for the initial question of what mechanisms in sport could explain the association between participation and positive developmental outcomes. Goal setting and skill development were significantly associated with some of the PYD outcomes, which does provide some insight to the causal mechanisms. Yet, the proposed sport-based model was not supported. That said, there are lessons to be learned and avenues to pursue for future research in the area of sport-based positive youth development.

A particular need is for longitudinal study designs and person-centered approaches. Study Three was a single time point, cross-sectional design, negating the ability to assess development over time. The associations between coaches, teammates, experiences, and PYD outcomes may be better assessed through longitudinal, multiple 
time point study designs to test change, and trajectories of change, over time. In addition, incorporating a person-centered approach to capture development would better elucidate the nature of the associations between PYD climate, experiences, and PYD outcomes.

Ullrich-French and Cole (2018) recently conducted a study looking at the Girls on the Run after-school program, a PYD-based physical activity training program. Taking a person-centered approach, the authors were able to identify developmental patterns based on clusters. More specifically, results showed that participants who were classified as "low starters" (i.e., were low on each of the outcomes at the first time point) showed the greatest change across time whereas "high starters" showed no significant change and even a decline in some cases. The outcomes assessed were self-worth, perceptions of physical competence, and emotional self-efficacy. In another person-centered study, Vierimaa et al., (2018) found that participants high in the 5 Cs reported more communication with their coaches compared to those in the low $5 \mathrm{C}$ cluster. This, again, provides a slightly more nuanced perspective of the association between the sport climate (e.g., relationship with coaches) and PYD outcomes.

A person-centered approach, like the one employed by the researchers mentioned, may be particularly important for assessing PYD and for determining if sport provides a context for development. By observing trajectories, or assessing within-person change, a more nuanced perspective can be analyzed. As development implies change over a given time period, it may be that specific associations and the nature of these associations cannot be accurately captured at a single time point. In addition, those who are already high in a specific PYD outcome may not show the same trajectory as those who are lower on PYD outcomes. Those who begin lower may show greater gains that would not be 
detected without assessing individual-level changes. Adversity may represent one factor that would be more accurately captured using a longitudinal, person-centered approach. Neely et al., (2018) recently reported that the experience of being deselected (or "cut") from a team took time for athletes to process. Therefore, what is gained from adverse experience may take time to capture, as would be possible through a person-centered, longitudinal design.

\section{Limitations}

As with all research, there are additional caveats to address with Study Three. Primarily, the sample was drawn from a university population. The sample was restricted based on age and the mean age was within the late adolescent developmental time period. As mentioned, adolescence generally refers to the developmental period from ten to 19 (although there are arguments being made that this should be extended into the $20 \mathrm{~s}$; e.g., Sawyer et al., 2018). The original data collection plan included participants from ages 13 to 19. Unfortunately, the COVID 19 epidemic in Canada prevented the collection of data in high schools and with youth sports teams. Future research should incorporate participants from early and mid-adolescence to evaluate the sport-based model within that developmental time frame. Additionally, variability in socio-demographic variables should be considered to understand how social factors influence the sport context and to understand how experiences differ based on things like socio-economic status, geographic location, and availability of sport programs.

Sample size may have limited the power and ability of statistical tests to obtain significant results for the overall model. Although the number of participants met the general minimum requirements for structural equation modeling, additional participants 
would have provided additional information to the overall model. Future research should attempt to increase sample size and recruit participants from a variety of sport environments.

Some limitations with regard to the design of the ESSY are important to recognize. One limitation is that the goal setting factor in the ESSY contained more items (9 items) than the adversity (5 items) or skill development (5 items) factors. It is possible that the additional information provided by these items contributed to the significant findings compared to the other two factors. Another key limitation is the mismatch between the question stem and the response options. Participants were asked to identify how closely the items represented them and their experiences in sport and the response options ranged from 'never' to 'always.' For some items, the response options may not have been consistent with what the question was asking, for example, I have had the opportunity to develop a wide range of physical skills. Such an item does not necessarily lend itself to a response based on frequency of occurrence. This type of mismatch has been shown to reduce data quality (Smyth \& Olson, 2019). Alternate response options (e.g., not at all like me to very much like me) or question stems (e.g., how frequently does this happen to you when you play sports?) should be explored if the ESSY is to be used to assess experiences in sport. Along this vein, continued validation of the ESSY is needed to confirm that it is a valid and reliable measure of adolescent experiences in sport. This measure was developed to address a gap in the literature and may be valuable in capturing a currently missing component of the understanding of PYD in sport, but additional work is needed to ensure it is a valid and reliable indicator of experiences in sport. 


\section{Conclusion}

This study set out to validate the ESSY and test the adapted sport-based model of PYD. Findings confirmed the factor structure of the ESSY, suggesting that it can provide a reliable way to assess youth experiences in sport. Hypotheses were only partially supported for model testing and showed goal setting to be most strongly associated with PYD outcomes. Despite the limitations, results provide insight into the value of goal setting for youth experiences in sport and adds to the current knowledge base.

\section{General Discussion}

The overarching purpose of this study was to create a measure to assess developmentally relevant experiences in sport and to test this measure using the Sportbased Model of PYD. Using a rational-theoretical approach to scale development and incorporating interviews and expert reviews, the Experience in Sport Scale for Youth provided a way to assess experiences of goal setting, adversity, and skill development in sport. This measure was then used to test a sport-based model of PYD, based on the sport-based model proposed by Holt et al., (2017). It was expected that the PYD climate, as measured by coach and peer relationships, would be positively associated with experiences in sport (goal setting, adversity, skill development), which would then be positively associated with the 5 Cs of PYD (competence, confidence, character, caring, and connection). These expectations were only partially supported.

The sport-based model proposed by Holt et al., (2017) was supported by qualitative findings in Study One but not statistically in Study Three. Findings from the qualitative study were interesting in their consistency with other qualitative work, including the studies summarized in the development of the Sport-Based Model of PYD. 
The value placed on these relationships in sport and the fact that these relationships create the context in which sport is played is one example of this congruency. For example, enjoyment in sport was very much influenced by relationships with peers and coaches. These relationships were noted as some of the key reasons for engaging in sport and, particularly, in the continuance of participation in sport as an adult. Without these positive relationships, it appears unlikely that sport would remain a meaningful activity for many participants. Furthermore, as previously discussed, a salient experience with a coach was relayed by each of the participants, some negative and some positive. Yet, when these associations were tested in Study Three, the PYD climate did not significantly predict PYD outcomes.

There are a few postulations as to why the model did not fit. These reasons will be discussed in detail in the following sections but, generally, pertain to the conceptualization and measurement of PYD in sport. Overall, this thesis did provide some interesting findings to add to the knowledge base. The ESSY, as a measure of experiences in sport, was developed and the factor structure was confirmed. Additional work is needed to further validate the ESSY as a measure of experiences in sport, but future research can build off of this initial step with the ESSY. Furthermore, goal setting and skill development were shown to be valuable to PYD outcomes with goal setting being positively associated with each of the 5 Cs. Again, future work can incorporate this information to further develop the concept of PYD in sport.

\section{Assessing PYD in Sport}

Within the positive youth development literature, PYD has been conceptualized in different ways. The $5 \mathrm{C}$ conceptualization is, arguably, the most well-known and the most 
tested model. Lerner, Lerner and colleagues (2005) took the concept of PYD and developed a way to operationalize each construct, making it testable. That is, they developed a measure that operationalizes PYD as competence, confidence, character, caring, and connection. Although the original measure is quite long, the measure used in Study Three is concise, meaning that it has an added benefit of reducing demand on participants. Overall, it is an appealing conceptualization for conducting research. The 5 Cs conceptualization was selected for this study because it represented a well-known and well-researched concept within the PYD literature. In addition, the 5 Cs were tangible outcomes that were consistent with the outcomes described by Holt et al., (2017), in the PYD outcomes of the Sport-Based Model of PYD (e.g., the social category is captured by connectedness). Nevertheless, the 5 Cs may not be fully capturing PYD in sport.

The findings from the present thesis, and developmental theory in general, may be better aligned with the way in which Larson has conceptualized PYD outcomes. Autonomy, agency, and being an active player in development are key elements of the Larson approach (Larson, 2006). The factors of the ESSY all speak to these in some way. Goal setting, for the most part, comes from within and requires the individual to develop their own sense of purpose (what they want to achieve) and direct behaviours to work towards achieving that purpose (Larson, 2011). Skill development was conceptualized in such a way as to tap into aspects of agency and autonomy. For example, one item states, "I have worked hard to develop a specific skill." This statement implies that the individual has actively engaged in processes to improve. Characteristics like autonomy and agency may be more abstract concepts and can be interpreted in different ways depending on the developmental period in question. For example, agency can be 
evidenced through moral reasoning during the adolescent time frame (Sokol et al., 2015). That said, agency is an interactive and dynamic concept that develops through interactions with the environment (Sokol et al., 2015). This may make it an ideal characteristic for assessing changes in developmental outcomes over time and in an environment like sport.

The 5 Cs broadly capture many of the outcomes that PYD contexts are postulated to target, including social skills, developing connections with others, moral development, competence, etc. However, the broad categories of competence, confidence, character, caring, and connection may be too general in their scope and lack the specificity needed to understand how/if sport has a positive impact on development. Relatedly, the present study may have benefitted from an intermediate step between experiences and PYD outcomes. As was mentioned by participants in the interviews, and in numerous qualitative studies, participants learn valuable "life skills" through sport. These include things like time management, emotion regulation, and working with others. Perhaps, experiences in sport help develop these skills, which in turn are associated with PYD outcomes like agency. This would be more in line with the explicit pathway in the SportBased Model of PYD (see Figure 1).

The Sport-Based Model of PYD provided a valuable starting point for assessing developmental outcomes in the sport environment. Numerous qualitative studies have demonstrated the value placed on sport experiences by parents and coaches (Gould et al., 2007; Camiré et al., 2012; Neely \& Holt, 2014). Yet, quantitative work has not been able to fully capture the nature of these associations statistically. While there may be a myriad of reasons for why this may be the case, one reason addressed in the discussion for Study 
three was the need to consider longitudinal and person-centered approaches in PYD research.

The complexity of the sport environment and interaction of variables needs to be more holistically examined, modeled, and tested if the understanding of PYD in sport is to be assessed quantitatively. The ability to quantify effects within the sport environment has important implications for the understanding of sport-based PYD. Quantifiable values provide a mode for comparison of things like level of play, different types of sport, sex and/or gender effects, as well as providing comparison points for interventions or producing standards of practice.

\section{Individual Differences}

The Sport-Based Model of PYD developed by Holt et al (2017) does include the individual (see Figure 1) but the concept is external to the path models. In addition, the individual appears to influence the PYD climate, but that association is further influenced by distal (societal level) factors. Holt et al., note that, due to lack of research on the individual in qualitative sport research, the individual was not incorporated into the explicit or implicit path models. However, the authors do state in their discussion that research is needed in the area of individual differences and their impact on development outcomes.

Empirically testing the explicit pathway of the Sport-Based Model of PYD was the main objective of Study Three, thus individual characteristics were not assessed. Nevertheless, individual characteristics are likely to play a role in how sport experiences are perceived, how interactions with coaches/peers are perceived, and how PYD is realized in sport. Individual characteristics could include concepts like enjoyment, 
motivation, and sport orientation. To that point, a study by Hill (2013) found that win orientation moderated the association between teammate acceptance and symptoms of depression and anxiety, such that team acceptance did not predict anxiety/depression for female adolescent soccer players who were high in win orientation. What these findings indicate is that individual characteristics can play a role in how salient relationships and experiences are and how they impact key outcomes.

The purpose of organized sport varied for participants in the interviews. Some were in it for the social interaction, some liked the physical fitness that came along with it, and some liked the competitive parts. Someone who is participating in sports because they enjoy the social aspect and are focused on developing personal relationships, may have a different approach to experiences in the sport environment than someone who is intent on winning at all costs. For example, achievement motivation literature suggests that individuals who are task-involved are less focused on achievement over others and more focused on demonstrating improvement (Roberts et al., 2012).

The achievement orientation literature provides some interesting insights into how individual differences/characteristics might impact experiences in sport. Cetinkalp and Turksoy (2011) found that task orientation significantly predicted skill development as the main reason for sport participation in a group of adolescent male soccer players in Turkey. Furthermore, Vink et al., (2015) discovered a reciprocal association between intrinsic motivation and deliberate practice, such that initial levels of intrinsic motivation predicted future deliberate practice. Deliberate practice then predicted future levels of intrinsic motivation. Finally, goal orientation was found to be a strong predictor of confidence in a group of adolescent female volleyball players (Maygar \& Feltz, 2003). 
Even more interesting is the fact that this association was partially mediated by a coachestablished mastery climate (Magyar \& Feltz, 2003). Each of these studies demonstrate how an individual's achievement orientation is associated with various facets of the sport environment, including motivations (playing sports to develop skills), skill development (deliberate practice), coach climate, and outcomes (e.g., confidence).

Personality psychology offers another perspective on individual differences in sport. Research looking at personality and sport suggests that elite and team athletes are higher in extraversion scores (e.g., Allen et al., 2013) and extraversion has been linked to coach-athlete relationships, with similarities in level of extraversion related to a stronger relationship (and vice versa; Jackson et al., 2011). Such findings could have implications for perceptions of the PYD climate and how the climate may differ for athletes who are introverted versus extroverted.

Research indicates that participation in sport can be beneficial for youth who are shy to enhance self-esteem and reduce social anxiety (Findlay \& Coplan, 2008). Yet, research from classroom settings shows that shyness can impact the quality of the student-teacher relationship (Rudasill, 2011; Zee \& Roorda, 2018). For example, Zee and Roorda (2018) found that, after controlling for teacher gender and experience, higher shyness scores were associated with lower relationship scores. Extending this to the sport setting, athletes who are shy may not have the same relationship with their coach which could impact perceptions of the PYD climate and mitigate the impact of a positive climate on PYD outcomes. Ultimately, assessing individual characteristics would create a more holistic understanding of PYD in sport.

\section{Systems at Play in the Sport Environment}


Developmental contexts are filled with interconnected and mutually influential factors. Based on the findings from each of the studies in this thesis, this section will address some of the systemic factors at play and discuss what can be gleaned from the findings as well as considerations for future work.

\section{Level of Play}

The differences between goal setting and skill development scores for recreational players and competitive players in organized sport in Study Three are noteworthy. Both experiences with goal setting and skill development were higher for competitive players. Most of the sport research has been conducted with elite athletes making it difficult to ascertain if specific outcomes are associated with organized sport, generally, or with elite and/or competitive level athletics, in particular. It makes sense that competitive players report more experiences with skill development as they typically have more practice times and games in a given week, month, or season. The goal of competitive and elite level sports is to win and in order to win, one needs to be better than the competition. Thus, emphasis on skill development and time devoted to skill development would be higher in competitive sports. Understanding how level of play may be differentially associated with PYD outcomes will be an important part of future PYD research.

As the intent was to capture a wide range of experiences, the type of sport was not restricted in Study Three leading to a wide variety in the type of sports reported by participants. Future research would benefit from looking at specific sports and definitive levels of play to be able to capture differences between sports and levels of play. Level of play was self-reported and categorized as either competitive or recreational. Within the competitive sport realm, there are a myriad of levels and these can differ between sports. 
In addition, the characteristics associated with the environments may also play a role in experiences. For example, the experiences on a competitive dance team may be different than the experiences on a competitive hockey team.

\section{Coaching, Coach Quality, and Individual Preferences}

Coaching and coach behaviours are fundamental to the youth experience. This was shown in the interviews in Study One and has been repeatedly demonstrated in both qualitative (Gould et al., 2007; McCarthy \& Jones, 2007) and quantitative studies (Coatsworth \& Conroy, 2006; Mouratidis et al., 2010). With most youth sports, coaches are parent volunteers that must take training courses to become certified. The level of certification required increases as the level of play increases (see Canada Basketball, 2021; Hockey Canada, 2021). Thus, the quality of coach is also relative to the level of play. This could mean that those who play competitive sports are not merely getting more time in their sport, they are also getting a higher quality of coach with skills to encourage development in specific areas, such as goal setting and skill development. Life-skill transfer research and research on the explicit pathway of PYD have shown program quality (Bean, Harlow, et al., 2018) and coaches, in particular, to be key in the transfer of skills in PYD programs (e.g., Bean, Kramers, et al., 2018). This, again, points to the interactive nature of sport contexts and the importance of considering how these systems interact to predict PYD.

As there are contextual differences that impact experiences with coaches, there are also individual differences in the perception of coach relationships and behaviour. Not everyone has the same experience with a coach and not everyone will perceive behaviour from the coach the same way. As reported by one participant in the interviews, 
a harsher approach was effective because the individual felt that was more motivating. Mental toughness research suggests that elite athletes prefer a harsher coaching approach for performance outcomes (Crust \& Azadi, 2009). Others may not feel that same sense and interpret harsh (e.g., yelling) behaviour as negative, detracting from the sport experience. How these individual differences interact to affect experiences and PYD outcomes will need to be assessed in future work.

The Sport-Based Model of PYD shows two potential pathways to PYD - the implicit and explicit pathways. The present study tested the implicit pathway whereby the natural interactions between the individual and the environment are expected to promote development. With the explicit pathway, programs are designed to target specific "life skills "and these are verbalized to program participants. This idea has recently been conceptualized as a continuum moving from implicit to explicit, depending on the level of focus on life skills and their transfer (see Bean, Kramers, et al., 2018). According to Bean, Kramers, et al., (2018), as coaches move along the continuum, PYD is optimized. The explicit pathway provides support for the hypothesis that coaches moderate the association between experiences in sport and PYD outcomes. As demonstrated by the sport-based model of PYD, coaches (and peers/parents) create the climate in which participants develop. This conceptualization does not, however, speak to the value of sport experiences for life skill development but may provide an insight into what can be assessed as outcomes in future PYD work.

\section{Society and the Changing Nature of Sport}

A discussion on organized sport would not be complete without acknowledging the current state of youth sport and the problems this could pose for PYD research. There 
is much more focus on specialization of sport with competitive athletes foregoing seasonal sports, or even multiple sports at one time, and focusing on one sport. One Canadian-based survey reported that $82 \%$ of respondents played hockey for more than half of the year (FlipGive, 2019). Emerging research indicates that this is a problematic trend for youth sport. Athletes are specializing at younger ages compared to current professional athletes (Buckley et al., 2017). These "early specializers" are more prone to injury (Bell et al., 2018) and burnout (Brenner et al., 2019) and may even be less likely to engage in sport as young adults (Russell \& Limle, 2013; Ullrich-French \& Cole, 2018) The implications and inequities that such sport models foster (e.g., cost of sport), are important areas for future sport-based PYD research and understanding differences in level of play and the associated outcomes. Early specialization may be structured to focus on skill and the development of the individual as an athlete and less so on developing an individual as a person. Understanding how these new models of sport participation impact psycho-social adolescent development will be an important future direction for PYD in sport.

The predominant theme of this thesis is the value of organized sport to positive developmental outcomes. However, as the cost of organized sports rises, the ability of youth to engage in a sport becomes more challenging for families. Consider that a recent report found that almost $90 \%$ of parents in Canada and the U.S. reported concerns about the impact of paying for hockey on their finances. Some parents even reported getting a second job (23\%) or taking on additional debt (35\%) to pay for their child to play hockey (FlipGive, 2019). In addition, another survey reported that the average family in Canada spends almost $\$ 1000$ per child on organized sports (Vital Signs, 2019). Reasons for 
supporting their children in organized sports were the strong beliefs that sport contributed to their child's positive development (e.g., learning teamwork, and improving school grades; FlipGive, 2019; Vital Signs, 2019). Parents see value in organized sport, but the costs may be untenable for some families. Understanding the socio-economic implications of participation in sport is important to ensure that sport remains a viable option for those who want to participate.

\section{An Interactive Model of PYD in Sport}

The developmental systems theory describes the interactive nature of development and the fact that development takes place as an individual interacts with people, places, and things in their environment. The reciprocity of this interaction is a key point to consider with regard to the outcome of this thesis. As mentioned, the complexity of the sport environment (as any developmental context) makes it challenging to test linear associations. So, too, does the reciprocity of developmental influence. Based on the above-mentioned concerns, the overall findings, and in-line with the literature, an adaptation of sport-based PYD is proposed in Figure 7. This new adaptation emphasizes the interactive and reciprocal nature of the sport climate. Within the sport environment, delineated by the rectangle, are the interactions between the individual, the climate, and experiences. The individual factors at play would be things like motivation and enjoyment but could extend to other characteristics, including personality. Both motivation and enjoyment have been shown to impact sport participation (Boiché \& Sarrazin, 2007; Gardner et al., 2017) and both were factors emphasized by interview participants as being critical to their experiences and to their continued participation. Relationships with parents, peers, and coaches are fundamental to participation in sport 
and remain within the PYD Climate, as originally outlined by Holt et al., (2017). The bidirectional association between these factors and the individual as well as between experiences highlights the complexity of these relationships. Finally, within the sport environment are the sport-specific experiences.

\section{Figure 7}

Interactive Model of Positive Youth Development Through Sport

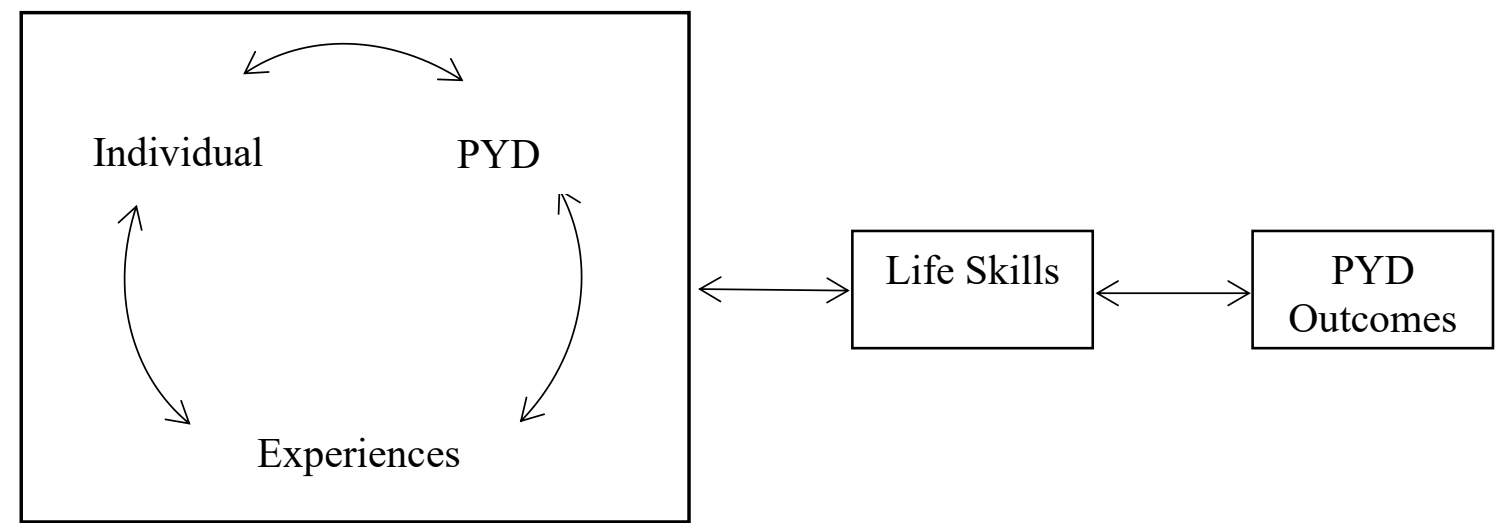

Time

The sport environment rectangle is indicative of the many systems that are at play in a sport context. Some of these may be out of the control of parents, coaches, and athletes and may reflect societal trends, such as costs associated with participation. As discussed, additional systems to consider are level of play, type of sport, and coach quality and could be variables that are controlled for or used to compare groups within the interactive model. These factors represent important and potentially defining characteristics of the sport context.

External to the environment are life skills as the proposed outcome of participation, in line with the explicit pathway within the Sport-Based Model of PYD. Life skills were mentioned by several interview participants as being valuable take-aways 
from sport. These included things like time management, emotion regulation, and teamwork, which have also been identified in the qualitative literature as key benefits of sport (e.g., Gould et al., 2007). PYD outcomes, as the ultimate goal of sport, are placed at the far right of the model. This is a higher-level factor and represents the epitome of development, which could include characteristics such as autonomy, competence, and self-regulation. The placement is indicative of the fact that development of these characteristics is dependent on each of the other variables in the model and takes time to develop. Furthermore, the bidirectional associations across the life skills and PYD outcomes indicate that the process is ongoing and iterative. Along the bottom of the figure, the time bar is representative of the development that is naturally occurring and necessary in the realization of PYD outcomes (e.g., time is needed to learn and practice skills).

The goal of this interactive model is not to present a Bronfenbrenner type model that is theoretically meaningful but unrealistic for research. This model is meant to provide a foundation so that researchers can elucidate the interactions that take place between variables in the sport context to promote developmental outcomes. This means that researchers should consider bidirectional associations and assessing trajectories of change in variables in study design and in statistical modelling.

\section{Limitations}

Several of the limitations have been addressed, but there are some points to reiterate. One of the key limitations of this thesis was the onset of the COVID-19 pandemic that shut down high schools and sports programs for several months in 2020 . This meant that the demographic of interest was no longer accessible, and the main 
method of data collection (in-person surveys) was no longer permissible. Recruiting online had been ongoing but was not continued or pursued after the onset of the epidemic in Canada as this was felt to not be appropriate given how challenging the experience has been, particularly for youth (Ellis et al., 2020).

Parents were not included in the testing of the sport-based model of PYD. This was done to focus on the factors that were in the immediate sport environment (i.e., involved on the playing surface). Interview participants were asked about the role their parents played in their sport experience and for the most part, it was as a chauffeur or cheerleader. Participants expressed gratitude for their parents support but parents did not come across as highly influential to the actual sport experience. Nevertheless, it would be prudent for future work to incorporate measures of relationships with parents.

Quantitative research has shown that parents have an impact on a variety of aspects in the sport experience including perceived competence (Horn \& Horn, 2007), psychosocial health (Van Yperen, 1998), and enthusiasm for sport (Power \& Woolger, 1994).

Obtaining perceptions of parent relationships from both the parent and child would be an important contribution for next steps in the PYD literature.

The Study Three discussion addressed the need to incorporate a longitudinal research design. A cross-sectional method was originally selected for this thesis for a few reasons. Firstly, previous research had employed this method successfully in various sport and PYD work. For example, Bloomfield and Barber (2011) and Larson et al (2006) used a cross-sectional design in their studies of developmental experiences in extracurricular activities, as did Vella et al (2013) in studying coaching and positive developmental experiences in soccer. Secondly, the aim of the study was to evaluate the 
association between PYD climate, PYD experiences, and PYD outcome variables and not to assess trajectories of development. Finally, there are pragmatic reasons for selecting a single time point design. As the pre-pandemic plan was to recruit from local high schools, it was expected that limiting the time demands on the principals, teachers, and students would encourage higher rates of participation. There are also fewer concerns with attrition and missing data with a single time-point approach. However, a cross-sectional design is limiting, particularly when looking at developmental outcomes.

\section{Strengths}

Despite some of the limitations, this thesis does add to the current sport-based PYD knowledge base. This thesis incorporated a multi-method approach to answer the question, "what is it about organized sports that make it a valuable context for positive youth development?" To date, a strong understanding of how specific experiences in sport are associated with PYD outcomes has been lacking. Using developmental theory and the Sport-Based Model of PYD proposed by Holt et al., (2017) as a base, a measure to assess experience in sport was developed. Adults and adolescents were interviewed to understand experiences in sport from those who were playing, or had played, organized sports and the themes uncovered were consistent with those proposed by the Sport-Based Model of PYD. Findings from the interviews and the literature were then used to develop the Experiences in Sport Scale for Youth (ESSY).

The ESSY underwent expert review before the factor structure was assessed using exploratory factor analysis. A 19-item, three factor ESSY was then tested using confirmatory factor analysis and the factor structure was confirmed. A simplified model of PYD in sport with experiential factors, as assessed by the ESSY, showed goal setting 
to be most strongly associated with confidence, competence, caring, character, and connection. Skill development was also significantly associated with character and caring. These findings provide support for examining experiences in sport and the potential importance of these experiences to positive developmental outcomes.

\section{Conclusion}

With the number of youth involved in sport and the apparent value to both physical and psychological health, understanding how to optimize the sport environment to promote positive developmental outcomes is important. This thesis sought to develop and test a measure of youth experiences in sport to better understand if experience represent a key construct within the Sport-Based Model of PYD. Although this thesis did not fully support the adapted Sport-Based Model of PYD, findings from the interviews did provide support for the importance of peers and coaches to the sport experience and structural equation modeling showed the value of goal setting and skill development to positive developmental outcomes. The concept of sport-based positive youth development is relatively new and there are a myriad of future directions to be taken. Future research can build on the findings from this thesis to develop a better understanding of how experiences in sport relate to developmental outcomes within the sport context. 


\section{References}

Abbott, B. D., \& Barber, B. L. (2011). Differences in functional and aesthetic body image between sedentary girls and girls involved in sports and physical activity: Does sport type make a difference? Psychology of Sport and Exercise, 12(3), 333-342. https://doi.org/10.1016/j.psychsport.2010.10.005

Adachi, P. J. C., \& Willoughby, T. (2014). It's not how much you play, but how much you enjoy the game: The longitudinal associations between adolescents' selfesteem and the frequency versus enjoyment of involvement in sports. Journal of Youth and Adolescence, 43(1), 137-145. https://doi.org/10.1007/s10964-0139988-3

Agans, J. P., Champine, R. B., DeSouza, L. M., Mueller, M. K., Johnson, S. K., \& Lerner, R. M. (2014). Activity involvement as an ecological asset: Profiles of participation and youth outcomes. Journal of Youth and Adolescence, 43(6), 919932. https://doi.org/10.1007/s10964-014-0091-1

Allen, J., \& Howe, B. L. (1998). Player ability, coach feedback, and female adolescent athletes' perceived competence and satisfaction. Journal of Sport and Exercise Psychology, 20(3), 280-299.

Al-Yaaribi, A., Kavussanu, M., \& Ring, C. (2016). Consequences of prosocial and antisocial behavior for the recipient. Psychology of Sport and Exercise, 26, 102 112. https://doi.org/10.1016/j.psychsport.2016.06.012

Amorose, A. J., \& Horn, T. S. (2001). Pre- to post-season changes in the intrinsic motivation of first year college athletes: relationships with coaching behavior and 
scholarship status. Journal of Applied Sport Psychology, 13(4), 355-373. https://doi.org/10.1080/104132001753226247

Baker, J., Côté, J., \& Hawes, R. (2000). The relationship between coaching behaviours and sport anxiety in athletes. Journal of Science and Medicine in Sport, 3(2), 110119. https://doi.org/10.1016/S1440-2440(00)80073-0

Baltes, P. B. (1987). Theoretical propositions of life-span developmental psychology: On the dynamics between growth and decline. Developmental Psychology, 23(5), 611-626. https://doi.org/10.1037/0012-1649.23.5.611

Balyi, P. D. I. (2001). Sport System Building and Long-term Athlete Development in British Columbia. Canada.

https://citeseerx.ist.psu.edu/viewdoc/download?doi=10.1.1.1058.7920\&rep=rep1 \&type $=\mathrm{pdf}$

Bandalos, D. L., \& Gerstner, J. (2016). Chapter three: Using factor analysis in test construction. In K. Schweizer \& C. DiStefano (Eds.) Principles and Methods of Test Construction (pp. 26-51). Hogrefe Publishing.

Bandura, A. (1997). Self-efficacy: The exercise of control (pp. ix, 604). W H Freeman/Times Books/ Henry Holt \& Co.

Bean, C., Harlow, M., Mosher, A., Fraser-Thomas, J., \& Forneris, T. (2018). Assessing differences in athlete-reported outcomes between high and low-quality youth sport programs. Journal of Applied Sport Psychology, 30(4), 456-472. https://doi.org/10.1080/10413200.2017.1413019

Bean, C., Kendellen, K., \& Forneris, T. (2016). Moving beyond the gym: Exploring life skill transfer within a female physical activity-based life skills program. Journal 
of Applied Sport Psychology, 28(3), 274-290.

https://doi.org/10.1080/10413200.2015.1124155

Bean, C., Kramers, S., Forneris, T., \& Camiré, M. (2018). The implicit/explicit continuum of life skills development and transfer. Quest, 70(4), 456-470. https://doi.org/10.1080/00336297.2018.1451348

Bell, D. R., Post, E. G., Biese, K., Bay, C., \& McLeod, T. V. (2018). Sport specialization and risk of overuse injuries: A systematic review with meta-analysis. Pediatrics, 142(3). https://doi.org/10.1542/peds.2018-0657

Benson, P. L. (2006). All kids are our kids: What communities must do to raise caring and responsible children and adolescents, 2nd ed (pp. xv, 429). Jossey-Bass.

Benson, P. L., Leffert, N., Scales, P. C., \& Blyth, D. A. (1998). Beyond the "village" rhetoric: Creating healthy communities for children and adolescents. Applied Developmental Science, 2(3), 138-159. https://doi.org/10.1207/s1532480xads0203_3

Biddle, S. J. H., \& Asare, M. (2011). Physical activity and mental health in children and adolescents: A review of reviews. British Journal of Sports Medicine, 45(11), 886-895. https://doi.org/10.1136/bjsports-2011-090185

Bigelow, B. J., Lewko, J. H., \& Salhani, L. (1989). Sport-involved children's friendship expectations. Journal of Sport \& Exercise Psychology, 11(2), 152-160.

Birkel, R. C., Lerner, R. M., \& Smyer, M. A. (1989). Applied developmental psychology as an implementation of a life-span view of human development. Journal of Applied Developmental Psychology, 10(4), 425-445. https://doi.org/10.1016/0193-3973(89)90020-8 
Blair, S. N., Cheng, Y., \& Holder, J. S. (2001). Is physical activity or physical fitness more important in defining health benefits? Medicine and Science in Sports and Exercise, 33(6), 379-399.

Blomfield, C. J., \& Barber, B. L. (2011). Developmental experiences during extracurricular activities and Australian adolescents' self-concept: Particularly important for youth from disadvantaged schools. Journal of Youth and Adolescence, 40(5), 582-594. https://doi.org/10.1007/s10964-010-9563-0

Boiché, J. C. S., \& Sarrazin, P. G. (2007). Self-determination of contextual motivation, inter-context dynamics and adolescents' patterns of sport participation over time. Psychology of Sport and Exercise, 8(5), 685-703. https://doi.org/10.1016/j.psychsport.2006.10.004

Bowers, E. P., Li, Y., Kiely, M. K., Brittian, A., Lerner, J. V., \& Lerner, R. M. (2010). The five Cs model of positive youth development: A longitudinal analysis of confirmatory factor structure and measurement invariance. Journal of Youth and Adolescence, 39(7), 720-735. https://doi.org/10.1007/s10964-010-9530-9

Bowker, A. (2006). The relationship between sports participation and self-esteem during early adolescence. Canadian Journal of Behavioural Science, 38(3), 214-229. https://doi.org/10.1037/cjbs2006009

Brenner, J. S., LaBotz, M., Sugimoto, D., \& Stracciolini, A. (2019). The psychosocial implications of sport specialization in pediatric athletes. Journal of Athletic Training, 54(10), 1021-1029. https://doi.org/10.4085/1062-6050-394-18

Bronfenbrenner, U., \& Morris, P. (1998). The ecology of developmental processes. In Theorectical models of human development (pp. 993-1028). Wiley. 
https://nyuscholars.nyu.edu/en/publications/the-ecology-of-developmentalprocesses

Bronfenbrenner, Urie. (1986). Ecology of the family as a context for human development: Research perspectives. Developmental Psychology, 22(6), 723-742. Bronfenbrenner, Urie. (2005). Making human beings human: Bioecological perspectives on human development. Sage Publications Ltd.

Brown, H. E., Lafferty, M. E., \& Triggs, C. (2015). In the face of adversity: Resiliency in winter sport athletes. Science \& Sports, 30(5), e105-e117. https://doi.org/10.1016/j.scispo.2014.09.006

Buckley, P. S., Bishop, M., Kane, P., Ciccotti, M. C., Selverian, S., Exume, D., Emper, W., Freedman, K. B., Hammoud, S., Cohen, S. B., \& Ciccotti, M. G. (2017). Early single-sport specialization: A survey of 3090 high school, collegiate, and professional athletes. Orthopaedic Journal of Sports Medicine, 5(7), 2325967117703944. https://doi.org/10.1177/2325967117703944

Bundick, M. J. (2011). Extracurricular activities, positive youth development, and the role of meaningfulness of engagement. The Journal of Positive Psychology, 6(1), 57-74. https://doi.org/10.1080/17439760.2010.536775

Burton, D., \& Weiss, C. (2008). The fundamental goal concept: The path to process and performance success. In Advances in sport psychology, 3rd ed (pp. 339-375,470474). Human Kinetics.

Cambridge Dictionary. (2021). Sport. https://dictionary.cambridge.org/dictionary/english/sport 
Camiré, M., Trudel, P., \& Forneris, T. (2012). Coaching and transferring life skills: philosophies and strategies used by model high school coaches. The Sport Psychologist, 26(2), 243-260. https://doi.org/10.1123/tsp.26.2.243

Canada Basketball. (2021). Canada Basketball | Coach. https://coach.ca/canadabasketball

Canada \& Canadian Heritage. (2014). Sport participation 2010-Research paper, June 2013. https://central.bac-lac.gc.ca/.item?id=CH24-1-2014eng\&op $=$ pdf\&app $=$ Library

Canadian Fitness and Lifestyle Research Institute. (2019). Bulletin 2: Participation in organized physical activity and sport | CFLRI. https://cflri.ca/bulletin-2participation-organized-physical-activity-and-sport-0

Canadian Society for Exercise Physiology. (2012). Canadian Physical Activity and Sedentary Behaviour Guidelines. http://www.csep.ca/cmfiles/guidelines/csep_guidelines_handbook.pdf

Carter, J. E., \& Rudd, N. A. (2005). Disordered eating assessment for college studentathletes. Women in Sport and Physical Activity Journal, 14(1), 62-71.

Cetinkalp, Z. K., \& Turksoy, A. (2011). Goal orientation and self-efficacy as predictors of male adolescent soccer players' motivation to participate. Social Behavior and Personality: An International Journal, 39(7), 925-935.

https://doi.org/10.2224/sbp.2011.39.7.925

Chaddock, L., Erickson, K. I., Prakash, R. S., Voss, M. W., VanPatter, M., Pontifex, M. B., Hillman, C. H., \& Kramer, A. F. (2012). A functional MRI investigation of the association between childhood aerobic fitness and neurocognitive control. 
Biological Psychology, 89(1), 260-268.

https://doi.org/10.1016/j.biopsycho.2011.10.017

Chaddock-Heyman, L., Erickson, K. I., Voss, M. W., Knecht, A. M., Pontifex, M. B., Castelli, D. M., Hillman, C. H., \& Kramer, A. F. (2013). The effects of physical activity on functional MRI activation associated with cognitive control in children: A randomized controlled intervention. Frontiers in Human Neuroscience, 7. https://doi.org/10.3389/fnhum.2013.00072

Chard, C., Edwards, K. I., \& Potwarka, L. (2015). Understanding the perceived attributes and consequences of participation in youth "rep" hockey. Journal of Applied Sport Management, 7(2), 20-38.

Charmaz, K. (2014). Constructing grounded theory. SAGE.

Charmaz, K. (2006). Constructing Grounded Theory: A Practical Guide through Qualitative Analysis. SAGE.

Child, D. (2006). The Essentials of Factor Analysis. A\&C Black.

Clark, L. A., \& Watson, D. (2016). Constructing validity: Basic issues in objective scale development (p. 203). American Psychological Association. https://doi.org/10.1037/14805-012

Coatsworth, D., \& Conroy, D. E. (2006). Enhancing the self-esteem of youth swimmers through coach training: Gender and age effects. Psychology of Sport and Exercise, 7(2), 173-192. https://doi.org/10.1016/j.psychsport.2005.08.005

Coatsworth, J. D., \& Conroy, D. E. (2009). The effects of autonomy-supportive coaching, need satisfaction, and self-perceptions on initiative and identity in youth 
swimmers. Developmental Psychology, 45(2), 320-328.

https://doi.org/10.1037/a0014027

Conway, R. J., Heary, C., \& Hogan, M. J. (2015). An Evaluation of the measurement properties of the five Cs model of positive youth development. Frontiers in Psychology, 6. https://doi.org/10.3389/fpsyg.2015.01941

Costello, A. B., \& Osborne, J. (2005). Best practices in exploratory factor analysis: Four recommendations for getting the most from your analysis. Practical Assessment, Research and Evaluation, 10(7), 1-9. https://doi.org/10.7275/JYJ1-4868

Crocetti, E., Erentaitė, R., \& Žukauskienè, R. (2014). Identity styles, positive youth development, and civic engagement in adolescence. Journal of Youth and Adolescence, 43(11), 1818-1828. https://doi.org/10.1007/s10964-014-0100-4

Cronin, L. D., \& Allen, J. B. (2015). Developmental experiences and well-being in sport: The importance of the coaching climate. The Sport Psychologist, 29(1), 62-71. https://doi.org/10.1123/tsp.2014-0045

Crust, L., \& Azadi, K. (2009). Leadership preferences of mentally tough athletes. Personality and Individual Differences, 47(4), 326-330. https://doi.org/10.1016/j.paid.2009.03.022

Daniels, E., \& Leaper, C. (2006). A longitudinal investigation of sport participation, peer acceptance, and self-esteem among adolescent girls and boys. Sex Roles, 55(11), 875-880. https://doi.org/10.1007/s11199-006-9138-4

Danielsen, A. G., Breivik, K., \& Wold, B. (2011). Do perceived academic competence and school satisfaction mediate the relationships between perceived support provided by teachers and classmates, and academic initiative? Scandinavian 
Journal of Educational Research, 55(4), 379-401.

https://doi.org/10.1080/00313831.2011.587322

Danielsen, A. G., Wiium, N., Wilhelmsen, B. U., \& Wold, B. (2010). Perceived support provided by teachers and classmates and students' self-reported academic initiative. Journal of School Psychology, 48(3), 247-267. https://doi.org/10.1016/j.jsp.2010.02.002

Danish, S. J., Taylor, T. E., \& Fazio, R. J. (2003). Enhancing adolescent development through sports and leisure. In Blackwell handbook of adolescence (pp. 92-108). Blackwell Publishing.

Davis, C. L., Tomporowski, P. D., McDowell, J. E., Austin, B. P., Miller, P. H., Yanasak, N. E., Allison, J. D., \& Naglieri, J. A. (2011). Exercise improves executive function and achievement and alters brain activation in overweight children: A randomized, controlled trial. Health Psychology, 30(1), 91-98. https://doi.org/10.1037/a0021766

Dawes, N. P., \& Larson, R. (2011). How youth get engaged: Grounded-theory research on motivational development in organized youth programs. Developmental Psychology, 47(1), 259-269. https://doi.org/10.1037/a0020729

de Bruin, A. P., Oudejans, R. R., \& Bakker, F. C. (2007). Dieting and body image in aesthetic sports: A comparison of Dutch female gymnasts and non-aesthetic sport participants. Psychology of Sport and Exercise, 8(4), 507-520.

De Mello, M. T., Lemos, V. de A., Antunes, H. K. M., Bittencourt, L., Santos-Silva, R., \& Tufik, S. (2013). Relationship between physical activity and depression and 
anxiety symptoms: A population study. Journal of Affective Disorders, 149(1-3), 241-246. https://doi.org/10.1016/j.jad.2013.01.035

DeVellis, R. F. (2017). Scale Development: Theory and Applications-Robert F. DeVellis—Google Books (4th ed.). SAGE Publications, Inc.

Dorsch, T. E., Smith, A. L., Wilson, S. R., \& McDonough, M. H. (2015). Parent goals and verbal sideline behavior in organized youth sport. Sport, Exercise, and Performance Psychology, 4(1), 19-35. https://doi.org/10.1037/spy0000025

Dunn, A. L., Trivedi, M. H., Kampert, J. B., Clark, C. G., \& Chambliss, H. O. (2005). Exercise treatment for depression. American Journal of Preventive Medicine, 28(1), 1-8. https://doi.org/10.1016/j.amepre.2004.09.003

Eccles, J., \& Gootman, J. A. (2002). Community programs to promote youth development: Committee on community level programs for youth. National Academy Press.

Eccles, J. S., Barber, B. L., Stone, M., \& Hunt, J. (2003). Extracurricular activities and adolescent development. Journal of Social Issues, 59(4), 865-889. https://doi.org/10.1046/j.0022-4537.2003.00095.x

Eime, R. M., Young, J. A., Harvey, J. T., Charity, M. J., \& Payne, W. R. (2013). A systematic review of the psychological and social benefits of participation in sport for children and adolescents: Informing development of a conceptual model of health through sport. International Journal of Behavioral Nutrition and Physical Activity, 10(1), 98. https://doi.org/10.1186/1479-5868-10-98 
Elliot, A. J., \& Harackiewicz, J. M. (1994). Goal setting, achievement orientation, and intrinsic motivation: A mediational analysis. Journal of Personality and Social Psychology, 66(5), 968-980. https://doi.org/10.1037/0022-3514.66.5.968

Ellis, W. E., Dumas, T. M., \& Forbes, L. M. (2020). Physically isolated but socially connected: Psychological adjustment and stress among adolescents during the initial COVID-19 crisis. Canadian Journal of Behavioural Science, 52(3), 177187. https://doi.org/10.1037/cbs0000215

Emerson, J. A., \& Williams, D. M. (2015). The multifaceted relationship between physical activity and affect: Physical activity and affect. Social and Personality Psychology Compass, 9(8), 419-433. https://doi.org/10.1111/spc3.12190

Esteban-Cornejo, I., Gómez-Martínez, S., Tejero-González, C. M., Castillo, R., LanzaSaiz, R., Vicente-Rodríguez, G., Marcos, A., \& Martinez-Gomez, D. (2014). Characteristics of extracurricular physical activity and cognitive performance in adolescents. The AVENA study. Journal of Sports Sciences, 32(17), 1596-1603. https://doi.org/10.1080/02640414.2014.910607

Fabrigar, L. R., Wegener, D. T., MacCallum, R. C., \& Strahan, E. J. (1999). Evaluating the use of exploratory factor analysis in psychological research. Psychological Methods, 4(3), 272-299.

Fay, K. E. \& Lerner, R.M. (2013). Weighing in on the issue: A longitudinal analysis of the individual and contextual factors that influence the developmental trajectories of eating disorders among adolescents. Journal of Youth and Adolescence, 42(1), 33-51. doi: 10.1007/s10964-012-9844-x. 
Fereday, J., \& Muir-Cochrane, E. (2006). Demonstrating Rigor Using Thematic Analysis: A Hybrid Approach of Inductive and Deductive Coding and Theme Development. International Journal of Qualitative Methods, 8092. https://doi.org/10.1177/160940690600500107

Ferron, C., Narring, F., Cauderay, M., \& Michaud, P. A. (1999). Sport activity in adolescence: Associations with health perceptions and experimental behaviours. Health Education Research, 14(2), 225-233. https://doi.org/10.1093/her/14.2.225

Field, A. (2009). Discovering Statistics Using SPSS (Third). SAGE Publications, Inc.

Filby, W. C. D., Maynard, I. W., \& Graydon, J. K. (1999). The effect of multiple-goal strategies on performance outcomes in training and competition. Journal of Applied Sport Psychology, 11(2), 230-246.

https://doi.org/10.1080/10413209908404202

Findlay, L. C., \& Coplan, R. J. (2008). Come out and play: Shyness in childhood and the benefits of organized sports participation. Canadian Journal of Behavioural Science, 40(3), 153-161. http://dx.doi.org.proxy.library.carleton.ca/10.1037/0008400X.40.3.153

First Tee. (2021). About First Tee. First Tee. https://firsttee.org/about/

FlipGive. (2019). FlipGive \& Scotiabank report on the Real Cost of Hockey. https://www.flipgive.com/stories/flipgive-scotiabank-report-on-the-real-cost-ofhockey

Flora, D. B., LaBrish, C., \& Chalmers, R. P. (2012). Old and new ideas for data screening and assumption testing for exploratory and confirmatory factor analysis. Frontiers in Psychology, 3. https://doi.org/10.3389/fpsyg.2012.00055 
Ford, D. H., \& Lerner, R. M. (1992). Developmental systems theory: An integrative approach (pp. xi, 259). Sage Publications, Inc.

Forneris, T., Camiré, M., \& Williamson, R. (2015). Extracurricular activity participation and the acquisition of developmental assets: Differences between involved and noninvolved Canadian high school students. Applied Developmental Science, 19(1), 47-55. https://doi.org/10.1080/10888691.2014.980580

Fox, C. K., Barr-Anderson, D., Neumark-Sztainer, D., \& Wall, M. (2010). Physical activity and sports team participation: Associations with academic outcomes in middle school and high school students. The Journal of School Health, 80(1), 3137.

Franklin, S. B., Gibson, D. J., Robertson, P. A., Pohlmann, J. T., \& Fralish, J. S. (1995). Parallel Analysis: A method for determining significant principal components. Journal of Vegetation Science, 6(1), 99-106. https://doi.org/10.2307/3236261

Franzini, L., Elliott, M. N., Cuccaro, P., Schuster, Mark, Gilliland, M. J., Grunbaum, J. A., \& Tortolero, S. R. (2009). Influences of physical and social neighborhood environments on children's physical activity and obesity. American Journal of Public Health, 99(2), 271-278.

Fraser-Thomas, J., \& Côté, J. (2009). Understanding adolescents’ positive and negative developmental experiences in sport. Sport Psychologist, 23(1), 3-23.

Galli, N., \& Reed, J. (2012). Can good come from bad? An examination of adversarial growth in division I NCAA athletes. Journal of Intercollegiate Sport, 5(2), 199212. 
Galli, N., \& Vealey, R. S. (2008). “Bouncing back” from adversity: Athletes' experiences of resilience. The Sport Psychologist, 22(3), 316-335. https://doi.org/10.1123/tsp.22.3.316

Gallucci, N. T. (2014). Sport psychology: Performance enhancement, performance inhibition, individuals, and teams, 2nd ed (pp. viii, 679). Psychology Press.

Gardner, L. A., Magee, C. A., \& Vella, S. A. (2017). Enjoyment and behavioral intention predict organized youth sport participation and dropout. Journal of Physical Activity and Health, 14(11), 861-865. https://doi.org/10.1123/jpah.2016-0572

Gehlbach, H., \& Brinkworth, M. E. (2011). Measure twice, cut down error: A process for enhancing the validity of survey scales. Review of General Psychology, 15(4), 380-387. https://doi.org/10.1037/a0025704

Geldhof, G. J., Bowers, E. P., Mueller, M. K., Napolitano, C. M., Callina, K. S., \& Lerner, R. M. (2014). Longitudinal analysis of a very short measure of positive youth development. Journal of Youth and Adolescence, 43(6), 933-949. https://doi.org/10.1007/s10964-014-0093-z

Goldberg, J. H., \& King, Abby C. (2007). Physical Activity and Weight Management Across the Lifespan (SSRN Scholarly Paper ID 1077712). Social Science Research Network. https://papers.ssrn.com/abstract=1077712

Gould, D., Collins, K., Lauer, L., \& Chung, Y. (2007). Coaching life skills through football: A study of award winning high school coaches. Journal of Applied Sport Psychology, 19(1), 16-37.

Grady, M. P. (1998). Qualitative and Action Research: A Practitioner Handbook. Phi Delta Kappa International. 
Gulbin, J. P., Croser, M. J., Morley, E. J., \& Weissensteiner, J. R. (2013). An integrated framework for the optimisation of sport and athlete development: A practitioner approach. Journal of Sports Sciences, 31(12), 1319-1331. https://doi.org/10.1080/02640414.2013.781661

Hansen, D. M., \& Larson, R. (2005). The youth experience survey 2.0: Instrument revisions and validity testing. Unpublished Manuscript.

Hein, V., \& Hagger, M. (2007). Global self-esteem, goal achievement orientations, and self-determined behavioural regulations in a physical education setting. Journal of Sports Sciences, 25(2), 149-159.

Hill, K. (2013). My teammate, my peer: An examination of peer relationships within the context of sport [Master's thesis, Carleton University]. https://curve.carleton.ca/09c9eb97-9f21-483b-a523-74514572523f

Hillman, C. H., Erickson, K. I., \& Kramer, A. F. (2008). Be smart, exercise your heart: Exercise effects on brain and cognition. Neuroscience, 9, 58-65.

Hills, A. P., King, N. A., \& Armstrong, T. P. (2007). The contribution of physical activity and sedentary behaviours to the growth and development of children and adolescents. Sports Medicine, 37(6), 533-545.

Hockey Canada. (2021). NCCP | National Coaching Certification Program | hockey coach training. https://www.hockeycanada.ca/en-ca/hockeyprograms/coaching/essentials/nccp

Holt, N. L., Neely, K. C., Slater, L. G., Camiré, M., Côté, J., Fraser-Thomas, J., MacDonald, D., Strachan, L., \& Tamminen, K. A. (2017). A grounded theory of positive youth development through sport based on results from a qualitative 
meta-study. International Review of Sport and Exercise Psychology, 10(1), 1-49. https://doi.org/10.1080/1750984X.2016.1180704

Howells, K., \& Fletcher, D. (2015). Sink or swim: Adversity- and growth-related experiences in Olympic swimming champions. Psychology of Sport and Exercise, 16, 37-48. https://doi.org/10.1016/j.psychsport.2014.08.004

Hubley, A. M., \& Zumbo, B. D. (2013). Psychometric characteristics of assessment procedures: An overview. In APA handbook of testing and assessment in psychology, Vol. 1: Test theory and testing and assessment in industrial and organizational psychology (pp. 3-19). American Psychological Association. https://doi.org/10.1037/14047-001

Janssen, I., \& LeBlanc, A. G. (2010). Systematic review of the health benefits of physical activity and fitness in school-aged children and youth. International Journal of Behavioral Nutrition and Physical Activity, 7(1), 40. https://doi.org/10.1186/1479-5868-7-40

Jelicic, H., Bobek, D. L., Phelps, E., Lerner, R. M., \& Lerner, J. V. (2007). Using positive youth development to predict contribution and risk behaviors in early adolescence: Findings from the first two waves of the 4-H Study of Positive Youth Development. International Journal of Behavioral Development, 31(3), 263-273. https://doi.org/10.1177/0165025407076439

Jewett, R., Sabiston, C. M., Brunet, J., O’Loughlin, E. K., Scarapicchia, T., \& O’Loughlin, J. (2014). School sport participation during adolescence and mental health in early adulthood. Journal of Adolescent Health, 55(5), 640-644. https://doi.org/10.1016/j.jadohealth.2014.04.018 
Jõesaar, H., Hein, V., \& Hagger, M. S. (2012). Youth athletes' perception of autonomy support from the coach, peer motivational climate and intrinsic motivation in sport setting: One-year effects. Psychology of Sport and Exercise, 13(3), 257-262. https://doi.org/10.1016/j.psychsport.2011.12.001

Katzmarzyk, P. T., Tremblay, S., Morrison, R., \& Tremblay, M. S. (2007). Effects of physical activity on pediatric reference data for obesity. International Journal of Pediatroc Obesity, 2(3), 138-143.

Kelloway, E. K. (2014). Using MPlus for Structural Equation Modeling (Second). SAGE Publications, Inc.

Kim, Y., \& Lee, S. (2009). Physical activity and abdominal obesity in youth. Applied Physiology, Nutrition, and Metabolism, 34(4), 571-581.

Kingston, K. M., \& Wilson, K. M. (2008). The application of goal setting in sport. In Advances in Applied Sport Psychology (1st ed., pp. 85-133). Routledge. https://doi.org/10.4324/9780203887073-9

Kline, R. (2016). Principles and Practice of Structural Equation Modeling (Fourth). Guilford Press.

Koka, A., \& Hein, V. (2003). Perceptions of teacher's feedback and learning environment as predictors of intrinsic motivation in physical education. Psychology of Sport and Exercise, 4(4), 333-346. https://doi.org/10.1016/S1469-0292(02)00012-2

Krentz, E. M., \& Warschburger, P. (2013). A longitudinal investigation of sports-related risk factors for disordered eating in aesthetic sports. Scandinavian Journal of Medicine and Science in Sports, 23(3), 303-310. https://doi.org/doi: 10.1111/j.1600-0838.2011.01380.x. 
Kwan, M., Bobko, S., Faulkner, G., Donnelly, P., \& Cairney, J. (2014). Sport participation and alcohol and illicit drug use in adolescents and young adults: A systematic review of longitudinal studies. Addictive Behaviors, 39(3), 497-506. https://doi.org/10.1016/j.addbeh.2013.11.006

LaFontana, K. M., \& Cillessen, A. H. N. (2010). Developmental changes in the priority of perceived status in childhood and adolescence. Social Development, 19(1), 130-147. https://doi.org/10.1111/j.1467-9507.2008.00522.x

Larson, L., Reed W. (2000). Toward a psychology of positive youth development. American Psychologist, 55(1), 170-183. https://doi.org/10.1037/0003066X.55.1.170

Larson, R., \& Richards, M. H. (1991). Daily companionship in late childhood and early adolescence: Changing developmental contexts. Child Development, 62(2), 284300. https://doi.org/10.1111/j.1467-8624.1991.tb01531.x

Larson, R. W. (2006). Positive youth development, willful adolescents, and mentoring. Journal of Community Psychology, 34(6), 677-689. https://doi.org/10.1002/jcop.20123

Larson, R. W. (2011). Positive Development in a Disorderly World: Development in a disorderly world. Journal of Research on Adolescence, 21(2), 317-334. https://doi.org/10.1111/j.1532-7795.2010.00707.x

Larson, R. W., Hansen, D. M., \& Moneta, G. (2006). Differing profiles of developmental experiences across types of organized youth activities. Developmental Psychology, 42(5), 849-863. https://doi.org/10.1037/0012-1649.42.5.849 
Le Bars, H., Gernigon, C., \& Ninot, G. (2009). Personal and contextual determinants of elite young athletes' persistence or dropping out over time. Scandinavian Journal of Medicine and Science in Sports, 19(2), 274-285.

Lee, T. Y., Cheung, C. K., \& Kwong, W. M. (2012). Resilience as a positive youth development construct: A conceptual review. The Scientific World Journal, 2012, 390450. https://doi.org/10.1100/2012/390450

Lei, P. W., \& Wu, Q. (2007). Introduction to structural equation modeling: Issues and practical considerations. Educational Measurement: issues and practice, 26(3), $33-43$.

Leite, N. M. C., \& Sampaio, J. E. (2012). Long-term athletic development across different age groups and gender from Portuguese basketball players. International Journal of Sports Science \& Coaching, 7(2), 285-300.

https://doi.org/10.1260/1747-9541.7.2.285

Lerner, Richard M., Lerner, J. V., Almerigi, J. B., Theokas, C., Phelps, E., Gestsdottir, S., Naudeau, S., Jelicic, H., Alberts, A., Ma, L., Smith, L. M., Bobek, D. L., Richman-Raphael, D., Simpson, I., Christiansen, E. D., \& von Eye, A. (2005). Positive youth development, participation in community youth development programs, and community contributions of fifth-grade adolescents: Findings from the first wave of the 4-H study of positive youth development. The Journal of Early Adolescence, 25(1), 17-71. https://doi.org/10.1177/0272431604272461

Lerner, Richard M., Lerner, J. V., De Stefanis, I., \& Apfel, A. (2001). Understanding developmental systems in adolescence: Implications for methodological 
strategies, data analytic approaches, and training. Journal of Adolescent Research, 16(1), 9-27. https://doi.org/10.1177/0743558401161003

Lerner, Richard M., \& Overton, W. F. (2008). Exemplifying the integrations of the relational developmental system: Synthesizing theory, research, and application to promote positive development and social justice. Journal of Adolescent Research, 23(3), 245-255. https://doi.org/10.1177/0743558408314385

Lerner, Richard M., von Eye, A., Lerner, J. V., \& Lewin-Bizan, S. (2009). Exploring the foundations and functions of adolescent thriving within the 4-H Study of Positive Youth Development: A view of the issues. Journal of Applied Developmental Psychology, 30(5), 567-570. https://doi.org/10.1016/j.appdev.2009.07.002

Lerner, Richard M., Wang, J., Chase, P. A., Gutierrez, A. S., Harris, E. M., Rubin, R. O., \& Yalin, C. (2014). Using relational developmental systems theory to link program goals, activities, and outcomes: The sample case of the 4-H Study of Positive Youth Development: Sample case of the 4-H study of positive youth development. New Directions for Youth Development, 2014(144), 17-30. https://doi.org/10.1002/yd.20110

Lerner, R.M., \& Schmid Callina, K. (2014). The study of character development: Towards tests of a relational developmental systems model. Human Development, 57(6), 322-346. https://doi.org/10.1159/000368784

Lim, S., \& Jahng, S. (2019). Determining the number of factors using parallel analysis and its recent variants. Psychological Methods, 24(4), 452-467. https://doi.org/10.1037/met0000230 
Linden, C., Ahlborg, G., Besjakov, J., Gardsell, P., \& Karlsson, M. K. (2006). A school curriculum-based exercise program increases bone mineral accrual and bone size in prepubertal girls: Two-year data from the pediatric osteoporosis prevention (POP) study. Journal of Bone and Mineral Research, 21(6), 829-835.

Lindwall, M., Gerber, M., Jonsdottir, I. H., Börjesson, M., \& Ahlborg, G. (2014). The relationships of change in physical activity with change in depression, anxiety, and burnout: A longitudinal study of Swedish healthcare workers. Health Psychology, 33(11), 1309-1318. https://doi.org/10.1037/a0034402

Locke, E. A., \& Latham, G. P. (2006). New directions in goal-setting theory. Current Directions in Psychological Science, 15(5), 265-268.

Lubans, D. R., Morgan, P. J., Cliff, D. P., Barnett, L. M., \& Okely, A. D. (2010). Fundamental movement skills in children and adolescents. Sports Medicine, 40(12), 1019-1035. https://doi.org/10.2165/11536850-000000000-00000

Magyar, T. M., \& Feltz, D. L. (2003). The influence of dispositional and situational tendencies on adolescent girls' sport confidence sources. Psychology of Sport and Exercise, 4(2), 175-190. https://doi.org/10.1016/S1469-0292(01)00037-1

Mannes, M., Roehlkepartain, E. C., \& Benson, P. L. (2005). Unleashing the power of community to strengthen the well-being of children, youth, and families: An asset-building approach. Child Welfare: Journal of Policy, Practice, and Program, 84(2), 233-250.

Marcia, J. E. (1966). Development and validation of ego-identity status. Journal of Personality and Social Psychology, 3(5), 551-558. https://doi.org/10.1037/h0023281 
Markstrom, C. A., Li, X., Blackshire, S. L., \& Wilfong, J. J. (2005). Ego strength development of adolescents involved in adult-sponsored structured activities. Journal of Youth and Adolescence, 34(2), 85-95. https://doi.org/10.1007/s10964005-3208-8

Matsunaga, M. (2008). Item parceling in structural equation modeling: A primer. Communication Methods and Measures, 2(4), 260-293.

McCarthy, P. J., \& Jones, M. V. (2007). A qualitative study of sport enjoyment in the sampling years. The Sport Psychologist, 21(4), 400-416. https://doi.org/10.1123/tsp.21.4.400

McNeish, D. (2020). Should we use F-tests for model fit instead of chi-square in overidentified structural equation models? Organizational Research Methods, 23(3), 487-510. https://doi.org/10.1177/1094428118809495

Meade, A. W., \& Craig, S. B. (2012). Identifying careless responses in survey data. Psychological Methods, 17(3), 437-455. https://doi.org/10.1037/a0028085

Mellalieu, S., Hanton, S., \& O’Brien, M. (2013). The effects of goal setting on rugby performance. Journal of Applied Behaviour Analysis, 39(2), 257-261.

Moksnes, U. K., Moljord, I. E. O., Espnes, G. A., \& Byrne, D. G. (2010). Leisure time physical activity does not moderate the relationship between stress and psychological functioning in Norwegian adolescents. Mental Health and Physical Activity, 3(1), 17-22. https://doi.org/10.1016/j.mhpa.2009.12.002

Molenaar, P. C. M., Lerner, R. M., \& Newell, K. M. (2013). Handbook of Developmental Systems Theory and Methodology. Guilford Publications. 
Monteiro Gaspar, M. J., Amaral, T. F., Oliveira, B. M. P. M., \& Borges, N. (2011). Protective effect of physical activity on dissatisfaction with body image in children - A cross-sectional study. Psychology of Sport and Exercise, 12(5), 563569. https://doi.org/10.1016/j.psychsport.2011.05.004

Morgan, T., \& Giacobbi, P. (2006). Toward two grounded theories of the talent development and social support process of highly successful collegiate athletes. Sport Psychologist, 20(3), 295-313. https://doi.org/10.1123/TSP.20.3.295

Mouratidis, A., Lens, W., \& Vansteenkiste, M. (2010). How you provide corrective feedback makes a difference: The motivation role of communicating in an autonomy-supporting way. Journal of Sport and Exercise Psychology, 32(5), 619-637. https://doi.org/10.1123/jsep.32.5.619

Mueller, M. K., Phelps, E., Bowers, E. P., Agans, J. P., Urban, J. B., \& Lerner, R. M. (2011). Youth development program participation and intentional self-regulation skills: Contextual and individual bases of pathways to positive youth development. Journal of Adolescence, 34(6), 1115-1125. https://doi.org/10.1016/j.adolescence.2011.07.010

Nebel, S., Schneider, S., Beege, M., \& Rey, G. D. (2017). Leaderboards within educational videogames: The impact of difficulty, effort and gameplay. Computers \& Education, 113, 28-41.

https://doi.org/10.1016/j.compedu.2017.05.011

Neely, K. C., Dunn, J. G. H., McHugh, T.-L. F., \& Holt, N. L. (2018). Female athletes' experiences of positive growth following deselection in sport. Journal of Sport and Exercise Psychology, 40(4), 173-185. https://doi.org/10.1123/jsep.2017-0136 
Neely, K. C., \& Holt, N. L. (2014). Parents' perspectives on the benefits of sport participation for young children. The Sport Psychologist, 28(3), 255-268. https://doi.org/10.1123/tsp.2013-0094

Newman, T. J. (2020). Life skill development and transfer: “They're not just meant for playing sports.” Research on Social Work Practice, 30(6), 643-657. https://doi.org/10.1177/1049731520903427

Ntoumanis, N., Biddle, S. J. H., \& Haddock, G. (1999). The mediating role of coping strategies on the relationship between achievement motivation and affect in sport. Anxiety, Stress, \& Coping, 12(3), 299-327. https://doi.org/10.1080/10615809908250480

Ntoumanis, N., Healy, L. C., Sedikides, C., Duda, J., Stewart, B., Smith, A., \& Bond, J. (2014). When the going gets tough: The "why" of goal striving matters. Journal of Personality, 82(3), 225-236. https://doi.org/10.1111/jopy.12047

Ntoumanis, N., Taylor, I. M., \& Thøgersen-Ntoumani, C. (2012). A longitudinal examination of coach and peer motivational climates in youth sport: Implications for moral attitudes, well-being, and behavioral investment. Developmental Psychology, 48(1), 213-223. https://doi.org/10.1037/a0024934

O’Brien, M., Mellalieu, S., \& Hanton, S. (2009). Goal-setting effects in elite and nonelite boxers. Journal of Applied Sport Psychology, 21(3), 293-306.

https://doi.org/10.1080/10413200903030894

Ommundsen, Y., Roberts, G. C., Lemyre, P.-N., \& Miller, B. W. (2005). Peer relationships in adolescent competitive soccer: Associations to perceived 
motivational climate, achievement goals and perfectionism. Journal of Sports Sciences, 23(9), 977-989. https://doi.org/10.1080/02640410500127975

O’Rourke, D. J., Smith, R. E., Smoll, F. L., \& Cumming, S. P. (2014). Relations of parent- and coach-initiated motivational climates to young athletes' self-esteem, performance anxiety, and autonomous motivation: Who is more influential? Journal of Applied Sport Psychology, 26(4), 395-408. https://doi.org/10.1080/10413200.2014.907838

Partington, S., Partington, E., Heather, N., Longstaff, F., Allsop, S., Jankowski, M., Wareham, H., Stephens, R., \& Gibson, A. S. C. (2013). The relationship between membership of a university sports group and drinking behaviour among students at English Universities. Addiction Research \& Theory, 21(4), 339-347. https://doi.org/10.3109/16066359.2012.727508

Rasciute, S., \& Downward, P. (2010). Health or happiness? What is theiImpact of physical activity on the individual? International Review for Social Science, 63(2), 256-270. https://doi.org/10.1111/j.1467-6435.2010.00472.x

Rebar, A. L., Stanton, R., Geard, D., Short, C., Duncan, M. J., \& Vandelanotte, C. (2015). A meta-meta-analysis of the effect of physical activity on depression and anxiety in non-clinical adult populations. Health Psychology Review, 9(3), 366378. https://doi.org/10.1080/17437199.2015.1022901

Rhemtulla, M., Brosseau-Liard, P. É., \& Savalei, V. (2012). When can categorical variables be treated as continuous? A comparison of robust continuous and categorical SEM estimation methods under suboptimal conditions. Psychological Methods, 17(3), 354-373. https://doi.org/10.1037/a0029315 
Rich, G. J. (2003). The positive psychology of youth and adolescence. Journal of Youth and Adolescence, 32(1), 1-3. http://dx.doi.org.proxy.library.carleton.ca/10.1023/A:1021017421413

Roberts, G. C., Treasure, D. C., \& Conroy, D. E. (2012). Understanding the dynamics of motivation in sport and physical activity: An achievement goal interpretation. In Handbook of Sport Psychology (pp. 1-30). John Wiley \& Sons, Ltd. https://doi.org/10.1002/9781118270011.ch1

Roth, J. L., \& Brooks-Gunn, J. (2003). What exactly is a youth development program? Answers from research and practice. Applied Developmental Science, 7(2), 94111. https://doi.org/10.1207/S1532480XADS0702_6

Rudasill, K. M. (2011). Child temperament, teacher-child interactions, and teacher-child relationships: A longitudinal investigation from first to third grade. Early Childhood Research Quarterly, 26(2), 147-156. https://doi.org/10.1016/j.ecresq.2010.07.002

Russell, W. D., \& Limle, A. N. (2013). The relationship between youth sport specialization and involvement in sport and physical activity in young adulthood. Journal of Sport Behavior, 36(1), 82-98.

Santi, G., Bruton, A., Pietrantoni, L., \& Mellalieu, S. (2014). Full article: Sport commitment and participation in masters swimmers: The influence of coach and teammates. European Journal of Sport Science, 14(8), 852-860.

Sarkar, M., \& Fletcher, D. (2017). Chapter 10 - Adversity-related experiences are essential for Olympic success: Additional evidence and considerations. In V. 
Walsh, M. Wilson, \& B. Parkin (Eds.), Progress in Brain Research (Vol. 232, pp. 159-165). Elsevier. https://doi.org/10.1016/bs.pbr.2016.11.009

Sawyer, S. M., Azzopardi, P. S., Wickremarathne, D., \& Patton, G. C. (2018). The age of adolescence. The Lancet Child \& Adolescent Health, 2(3), 223-228. https://doi.org/10.1016/S2352-4642(18)30022-1

Scanlan, T. K., Carpenter, P. J., Simons, J. P., Schmidt, G. W., \& Keeler, B. (1993). An introduction to the sport commitment model. Journal of sport and exercise psychology, 15(1), 1-15.

Scarborough, M. K., Lewis, C. M., \& Kulkarni, S. (2010). Enhancing adolescent brain development through goal-setting activities. Social Work, 55(3), 276-278.

Schmid, K. L., Phelps, E., \& Lerner, R. M. (2011). Constructing positive futures: Modeling the relationship between adolescents' hopeful future expectations and intentional self regulation in predicting positive youth development. Journal of Adolescence, 34(6), 1127-1135. https://doi.org/10.1016/j.adolescence.2011.07.009

Schunk, D. H. (1983). Ability versus effort attributional feedback: Differential effects on self-efficacy and achievement. Journal of Educational Psychology, 75(6), 848856. https://doi.org/10.1037/0022-0663.75.6.848

Schunk, D. H. (2001). Social cognitive theory and self-regulated learning. In Selfregulated learning and academic achievement: Theoretical perspectives, 2 nd ed (pp. 125-151). Lawrence Erlbaum Associates Publishers.

Sibold, J., Edwards, E., Murray-Close, D., \& Hudziak, J. J. (2015). Physical activity, sadness, and suicidality in bullied US adolescents. Journal of the American 
Academy of Child \& Adolescent Psychiatry, 54(10), 808-815.

https://doi.org/10.1016/j.jaac.2015.06.019

Silbereisen, R. K., \& Lerner, R. M. (2007). Approaches to Positive Youth Development. SAGE.

Simms, L. J. (2008). Classical and modern methods of psychological scale construction. Social and Personality Psychology Compass, 2(1), 414-433. https://doi.org/10.1111/j.1751-9004.2007.00044.x

Slater, A., \& Tiggemann, M. (2006). The Contribution of physical activity and media use during childhood and adolescence to adult women's body image. Journal of Health Psychology, 11(4), 553-565. https://doi.org/10.1177/1359105306065016

Smith, A. L. (2003). Peer relationships in physical activity contexts: A road less traveled in youth sport and exercise psychology research. Psychology of Sport and Exercise, 4(1), 25-39. https://doi.org/10.1016/S1469-0292(02)00015-8

Smith, R. E., Cumming, S. P., \& Smoll, F. L. (2008). Development and validation of the motivational climate scale for youth sports. Journal of Applied Sport Psychology, 20(1), 116-136. https://doi.org/10.1080/10413200701790558

Smith, R. E., Smoll, F. L., \& Cumming, S. P. (2009). Motivational climate and changes in young athletes' achievement goal orientations, 33(2), 173-183. https://doi.org/10.1007/s11031-009-9126-4

Smoll, F. L., Smith, R. E., Barnett, N. P., \& Everett, J. J. (1993). Enhancement of children's self-esteem through social support training for youth sport coaches. Journal of Applied Psychology, 78(4), 602-610. https://doi.org/10.1037/00219010.78.4.602. 
Smyth, J. D., \& Olson, K. (2019). The effects of mismatches between survey question stems and response options on data quality and responses. Journal of Survey Statistics and Methodology, 7(1), 34-65.

Sokol, B. W., Hammond, S. I., Kuebli, J., \& Sweetman, L. (2015). The development of agency. In Handbook of Child Psychology and Developmental Science (pp. 1-39). American Cancer Society. https://doi.org/10.1002/9781118963418.childpsy108

Sport for Life. (2019). Physical literacy. Sport for Life. https://sportforlife.ca/physicalliteracy.

Stephens, L. J., \& Schaben, L. A. (2002). The Effect of interscholastic sports participation on academic achievement of middle level school students. NASSP Bulletin, 86(630), 34-41.

Strohle, A., Hofler, M., Pfister, H., Muller, A. G., Hoyer, J., Wittchen, H.-U., \& Lieb, R. (2007). Physical activity and prevalence and incidence of mental disorders in adolescents and young adults. Psychological Medicine, 37(11), 1657-1666.

Strong, W. B., Malina, R. M., Blimkie, C. J. R., Daniels, S. R., Dishman, R. K., Gutin, B., Hergenroeder, A. C., Must, A., Nixon, P. A., Pivarnik, J. M., Rowland, T., Trost, S., \& Trudeau, F. (2005). Evidence based physical activity for school-age youth. The Journal of Pediatrics, 146(6), 732-737. https://doi.org/10.1016/j.jpeds.2005.01.055

Stuart, M. E., \& Ebbeck, V. (1995). The influence of perceived social approval on moral development in youth sport. Pediatric Exercise Science, 7(3), 270-280. https://doi.org/10.1123/pes.7.3.270 
Swain, A., \& Jones, G. (1995). Effects of goal-setting interventions on selected basketball skills: A single-subject design. Research Quarterly for Exercise and Sport, 66(1), 51-63. https://doi.org/10.1080/02701367.1995.10607655

Tabachnick, B., \& Fidell, L. (2013). Using Multivariate Statistics (Sixth). Pearson.

Taliaferro, L. A., Eisenberg, M. E., Johnson, K. E., Nelson, T. F., \& Neumark-Sztainer, D. (2011). Sport participation during adolescence and suicide ideation and attempts. International Journal of Adolescent Medicine and Health, 23(1), 3-10.

Tavares, D., \& Freire, T. (2016). Flow experience, attentional control, and emotion regulation: Contributions for a positive development in adolescents. Revista PSICOLOGIA, 30(2), 77-94. https://doi.org/10.17575/rpsicol.v30i2.1119

Tenenbaum, G., \& Eklund, R. (2007). Handbook of Sport Psychology. John Wiley \& Sons.

Youngblade, L. M., \& Theokas, C. (2006). The multiple contexts of youth development: Implications for theory, research, and practice. Applied Developmental Science, $10(2), 58-60$.

Tomporowski, Phillip D., Davis, C. L., Miller, P. H., \& Naglieri, J. A. (2008). Exercise and children's intelligence, cognition, and academic achievement. Educational Psychology Review, 20(2), 111-131.

Tzetzis, G., Votsis, E., \& Kourtessis, T. (2008). The effect of different corrective feedback methods on the outcome and self confidence of young athletes. Journal of Sports Science \& Medicine, 7(3), 371-378.

Ullrich-French, S., \& Cole, A. N. (2018). Exploring participant characteristics in an assessment of changes in psychosocial outcomes in a physical activity-based 
positive youth development programme for girls. International Journal of Sport and Exercise Psychology, 16(5), 535-554.

https://doi.org/10.1080/1612197X.2016.1275740

Ullrich-French, S., \& Smith, A. L. (2006). Perceptions of relationships with parents and peers in youth sport: Independent and combined prediction of motivational outcomes. Psychology of Sport and Exercise, 7(2), 193-214. https://doi.org/10.1016/j.psychsport.2005.08.006

Ullrich-French, S., Smith, A. L., \& Cox, A. E. (2011). Attachment relationships and physical activity motivation of college students. Psychology \& Health, 26(8), 1063-1080. https://doi.org/10.1080/08870446.2010.530123

van de Pol, P. K. C., Kavussanu, M., \& Ring, C. (2012). Goal orientations, perceived motivational climate, and motivational outcomes in football: A comparison between training and competition contexts. Psychology of Sport and Exercise, 13(4), 491-499. https://doi.org/10.1016/j.psychsport.2011.12.002

Vazou, S., Ntoumanis, N., \& Duda, J. L. (2006). Predicting young athletes' motivational indices as a function of their perceptions of the coach- and peer-created climate. Psychology of Sport and Exercise, 7(2), 215-233. https://doi.org/10.1016/j.psychsport.2005.08.007

Vella, S. A., Oades, L. G., \& Crowe, T. P. (2013). The relationship between coach leadership, the coach-athlete relationship, team success, and the positive developmental experiences of adolescent soccer players. Physical Education \& Sport Pedagogy, 18(5), 549-561. https://doi.org/10.1080/17408989.2012.726976 
Vicente-Rodriguez, G., Ara, I., Perez-Gomez, J., Dorado, C., \& Calbet, J. a. L. (2005). Muscular development and physical activity as major determinants of femoral bone mass acquisition during growth. British Journal of Sports Medicine, 39(9), 611-616. https://doi.org/10.1136/bjsm.2004.014431

Vierimaa, M., Bruner, M. W., \& Côté, J. (2018). Positive youth development and observed athlete behavior in recreational sport. PLOS ONE, 13(1), e0191936. https://doi.org/10.1371/journal.pone.0191936

Vink, K., Raudsepp, L., \& Kais, K. (2015). Intrinsic motivation and individual deliberate practice are reciprocally related: Evidence from a longitudinal study of adolescent team sport athletes. Psychology of Sport and Exercise, 16, 1-6.

https://doi.org/10.1016/j.psychsport.2014.08.012

Vital Signs. (2019). Vital Signs: Sports and Belonging. https://communityfoundations.ca/wpcontent/uploads/2019/04/Vital_Signs_Sport_and_Belonging.pdf

Wadey, R., Clark, S., \& McCullough, D. (2013). Coaches' perceptions of athletes' stressrelated growth following sport injury. Psychology of Sport and Exercise, 14(2), $125-135$.

Wang, J., \& Wang, X. (2012). Structural Equation Modeling: Methods and Applications (First edition). John Wiley \& Sons Ltd.

Warburton, D. E. R., Nicol, C. W., \& Bredin, S. S. D. (2006). Health benefits of physical activity: The evidence. CMAJ: Canadian Medical Association Journal, 174(6), 801-809. https://doi.org/10.1503/cmaj.051351 
Watkins, M. W. (2005). Determining parallel analysis criteria. Journal of Modern Applied Statistical Methods, 5(2), 344-346. https://doi.org/10.22237/jmasm/1162354020

Watkins, M. W. (2018). Exploratory factor analysis: A guide to best practice. Journal of Black Psychology, 44(3), 219-246. https://doi.org/10.1177/0095798418771807

Watson, J. C., Connole, I., \& Kadushin, P. (2011). Developing young athletes: A sport psychology based approach to coaching youth sports. Journal of Sport Psychology in Action, 2(2), 113-122. https://doi.org/10.1080/21520704.2011.586452

Weinberg, R., Yukelson, D., Burton, D., \& Weigand, D. (2000). Perceived goal setting practices of Olympic athletes: An exploratory investigation. Sport Psychologist, 14, 279-295. https://doi.org/10.1123/tsp.14.3.279

Weiss, M. R., \& Duncan, S. C. (1992). The relationship between physical competence and peer acceptance in the context of children's sports participation. Journal of Sport \& Exercise Psychology, 14(2), 177-191.

Weiss, M. R., Kimmel, L. A., \& Smith, A. L. (2001). Determinants of sport commitment among junior tennis players: Enjoyment as a mediating variable. Pediatric Exercise Science, 13(2), 131-144. https://doi.org/10.1123/pes.13.2.131

Weiss, M. R., Smith, A. L., \& Theeboom, M. (1996). “That's what friends are for”: Children's and teenagers' perceptions of peer relationships in the sport domain. Journal of Sport and Exercise Psychology, 18(4), 347-379. https://doi.org/10.1123/jsep.18.4.347 
Weston, R., \& Gore Jr, P. A. (2006). A brief guide to structural equation modeling. The counseling psychologist, 34(5), 719-751.

Worthington, R. L., \& Whittaker, T. A. (2006). Scale development research: A content analysis and recommendations for best practices. The Counseling Psychologist, 34(6), 806-838. https://doi.org/10.1177/0011000006288127.

Wrosch, C., Scheier, M. F., \& Miller, G. E. (2003). Adaptive self-regulation of unattainable goals: Goal disengagement, goal reengagement, and subjective wellbeing. Personality and Social Psychology Bulletin, 29(12), 1494-1508.

Zee, M., \& Roorda, D. L. (2018). Student-teacher relationships in elementary school: The unique role of shyness, anxiety, and emotional problems. Learning and Individual Differences, 67, 156-166. https://doi.org/10.1016/j.lindif.2018.08.006.

Zhang, Y., \& Wildemuth, B. M. (2009). Qualitative analysis of content. Applications of social research methods to questions in information and library science, 308, 319.

Zimmerman, S. M., Phelps, E., \& Lerner, R. M. (2008). Positive and negative developmental trajectories in U.S. adolescents: Where the positive youth development perspective meets the deficit model. Research in Human Development, 5(3), 153-165. https://doi.org/10.1080/15427600802274001. 
Appendix A

\section{List of Preliminary ESSY Items}

\section{Goal Setting}

1. I had specific goals

2. I set long term goals

3. I set short term goals

4. I feel like the goals I set were challenging

5. I feel like the goals I set were achievable

6. I regularly reviewed my goals

7. I had specific plans/steps to achieve my goals

8. I told others (e.g., coaches, parents, teammates) about my goals

9. I have asked others for help to achieve my goals

10. Most of my goals were based on performance outcomes

11. I feel like I have achieved many of my goals

12. Most of my goals are based on skill development

\section{Adversity}

1. I have been cut from a team I wanted to make

2. I have (been part of a team that) lost a championship game

3. I have (been part of a team that) lost an important game

4. I have struggled to learn a new skill

5. I have played on a team where I have not gotten along with some people

6. I have disliked a coach

7. I have missed an important shot

8. I have suffered an injury that caused me to take time away from sports 
9. I felt like I made sacrifices to help my team

10. I have made a mistake that cost me or my team

11. I have felt nervous before a big game

12. I regularly felt nervous before I played

13. I have gotten so upset during a game that it affected my performance

14. I have gotten angry during a game

\section{Skill Development}

1. I feel like I had the opportunity to develop new skills

2. I found it easy to learn new skills

3. There was a lot of focus on skill development

5. I have worked hard to develop/improve a specific skill

6. I feel like I have developed a wide range of physical skills

7. I feel like I am good at sports

8. I had a lot of help from others to improve skills

9. I put a lot of effort into developing skills 
Appendix B

\section{Interview Protocol}

\section{Informed Consent}

1. Review consent and have participant sign. If under the age of 18, parent must provide consent.

\section{Interview Questions}

2. Background Information:
a. How old are you?
b. What sports did you play as a child/adolescent?
c. Did you play at a competitive or recreational level?
d. How long have you or did you play sports?
e. How did you start playing sports?

3. Tell me about your experiences playing sports.
a. When you think about playing sports, what comes to mind?
b. Did you enjoy playing sports?
c. Did you have a favourite sport?
d. What was your favourite part (or parts) of playing sports?
e. Are there ways in which you feel you benefited from playing sports?
f. If you played both competitive and recreational, were the experiences different? How did your experiences differ?

4. Did you have experiences in sports that you think really helped your development?
a. Can you describe your experiences with goal setting?
b. What are your feelings about facing adversity in sport?
c. Can you describe your experiences with skill development?

5. What role did your parents play in your sport experience?

6. How do you think your coaches affected your experience?

a. Can you describe a positive experience you had with a coach?

b. Can you describe a negative experience you had with a coach?

7. How do you think your teammates affected your experience? 
a. Can you describe a positive experience you had with a teammate?

b. Can you describe a negative experience you had with a teammate?

8. Is there anything else about your thoughts or experiences in sports that you would like to share?

\section{Additional Information}

1. Can you give me a pseudonym to use?

2. Would you like to receive a summary of the findings?

Debriefing - verbal and written debrief. 


\section{Appendix C}

\section{Participant Consent Form}

Title: Adolescent Experiences in Organized Sports

Date of Ethics Clearance: February 27 ${ }^{\text {th }}, 2018$

Ethics Clearance of the Collection of Data Expires: February $27^{\text {th }}, 2019$

I , choose to participate in a study on

experiences in organized sports. This study aims to understand the types of experiences adolescents have when playing organized sports. You will be asked questions designed to get you to reflect on the different types of experiences you had while playing organized sports in adolescence.

The researcher for this study is Kate Hill MacEachern in the Department of Psychology of Carleton University. She is working under the supervision of Anne Bowker, $\mathrm{PhD}$, in the Department of Psychology of Carleton University.

\section{Contact:}

Kate Hill MacEachern

Email: kate.hillmaceachern@,carleton.ca
Anne Bowker, $\mathrm{PhD}$

Anne.Bowker@carleton.ca

This study involves one, approximately, 60 minutes interview. With your consent, interviews will be audio-recorded. Once the recording has been transcribed, the audio recording will be destroyed.

This project will ask you about your experiences while playing organized sports. Some experiences can be negative and, as such, you may feel some discomfort in discussing these issues. Precautions will be taken to protect your identity and responses will be kept anonymous. In addition, you may request that certain responses not be included in the final project. Should you experience any discomfort during the interview, you will be provided with contact information for counseling services available to you.

You have the right to end your participation in the study at any time, for any reason. Any information you have provided will be destroyed immediately, should you choose to withdraw.

All research data, including audio-recordings and any notes will be encrypted. Any hard copies of data (such as handwritten notes or USB keys) will be kept in a locked cabinet at Carleton University. Research data will only be accessible by the researcher and the research supervisor.

Once the project is complete, all research data will be kept for five years and potentially used for other research projects on this same topic. At the end of five years, all research data will be securely destroyed.

If you would like a summary of the findings once the project is complete, you are invited to contact the researcher to request an electronic copy, which will be provided to you. 
The ethics protocol for this project was reviewed by the Carleton University Research Ethics Board, which provided clearance to carry out the research. If you have any ethical concerns with the study, please contact Dr. Andy Adler, Chair, Carleton University Ethics Board-B (by phone at 6136-520-2600 ext.4085 or via email at ethics@carleton.ca).

Do you agree to participate in this study?

Yes

No

Signature of participant:

Date:

Do you agree to be audio recorded?

Yes

No 


\section{Appendix D}

\section{Parent Consent Form}

Title: Adolescent Experiences in Organized Sports

Date of Ethics Clearance: February $27^{\text {th }}, 2018$

Ethics Clearance of the Collection of Data Expires: February $27^{\text {th }}, 2019$

I

, give permission for my child

to participate in a study about experiences

in organized sports. This study aims to understand the types of experiences adolescents have when playing organized sports. Your child will be asked questions about their time playing organized sports and the types if experiences they have had.

The researcher for this study is Kate Hill MacEachern in the Department of Psychology of Carleton University. She is working under the supervision of Anne Bowker, $\mathrm{PhD}$, in the Department of Psychology of Carleton University.

\section{Contact:}

Kate Hill MacEachern

Anne Bowker, $\mathrm{PhD}$

Email: kate.hillmaceachern@,carleton.ca

Anne.Bowker@carleton.ca

This study involves one, approximately, 60 minutes interview. With your consent, interviews will be audio-recorded. Once the recording has been transcribed, the audio recording will be destroyed.

This project will ask your child about the different types of experiences they have had while playing organized sports. These include such things as goal setting and skill development. However, some experiences may be negative and, as such, your child may feel some discomfort when discussing these issues. Precautions will be taken to protect your child's identity and responses will be kept anonymous. In addition, your child may request that certain responses not be included in the final project. Should your child experience any discomfort during the interview, she/he will be provided with contact information for counseling services and/or other resources will be made available to them.

Your child has the right to end his/her participation in the study at any time, for any reason. Any information they have provided will be destroyed immediately, should they choose to withdraw.

All research data, including audio-recordings and any notes will be encrypted. Any hard copies of data (such as handwritten notes or USB keys) will be kept in a locked cabinet at Carleton University. Research data will only be accessible by the researcher and the research supervisor. In cases where there is perceived to be serious risk to the participant (e.g. abuse, self-harm), the researcher has a duty to report this and cannot guarantee confidentiality.

Once the project is complete, all research data will be kept for five years and potentially used for other research projects on this same topic. At the end of five years, all research data will be securely destroyed. 
If you would like a summary of the findings once the project is complete, you are invited to contact the researcher to request an electronic copy, which will then be provided to you.

The ethics protocol for this project was reviewed by the Carleton University Research Ethics Board, which provided clearance to carry out the research. If you have any ethical concerns with the study, please contact Dr. Andy Adler, Chair, Carleton University Ethics Board-B (by phone at 6136-520-2600 ext. 4085 or via email at ethics@carleton.ca).

Do you agree to allow your child to participate in this study?

Yes

No

Signature of parent:

Date:

Do you agree for your child to be audio recorded?

Yes

No 
Appendix E

\section{Final Interview Protocol}

\section{Informed Consent}

1. Review consent and have participant sign. If under the age of 18, parent must provide consent. Will also ask for verbal consent.

Do you consent to participate in this study?

Do you consent to be audio-recorded.

*TURN ON audio recorder.

\section{Interview Questions}

2. Background Information:
a. How old are you?
b. Birthplace?
c. What sports did you play as a child/adolescent?
d. Did you play at a competitive or recreational level?
e. How long have you or did you play sports?
f. How did you start playing sports?

3. Tell me about your experiences playing sports.

a. When you think about playing sports, what comes to mind?

b. Did you enjoy playing sports?

c. Did you have a favourite sport?

i. What made it your favourite?

d. What was your favourite part (or parts) of playing sports?

e. Are there ways in which you feel you benefited from playing sports?

f. If you played both competitive and recreational, were the experiences different? How did your experiences differ?

4. Did you have experiences in sports that you think really helped your development?

a. Did/does sport take up a significant amount of time? Do you ever feel that it takes away from other areas of your life? Has sport interfered with personal commitments or experiences? 
b. Do you ever get nervous playing sports? Has playing sports helped you deal with nerves? Develop coping strategies?

c. Can you describe your experiences with goal setting?

i. Did you set goals?

ii. Did you achieve goals in sport?

d. What are your feelings about facing adversity in sport?

e. Can you describe your experiences with skill development?

i. Did you have the opportunity to work on weak skills during your organized practices? (e.g., wrong hand, wrong foot, crossovers)

ii. Do you feel like you have mastered skills?

iii. Did you work on skills during your own time (not during organized practices or games)

5. What role did your parents play in your sport experience?

6. How do you think your coaches affected your experience?

a. Can you describe a positive experience you had with a coach?

b. Can you describe a negative experience you had with a coach?

7. How do you think your teammates affected your experience?

a. Can you describe a positive experience you had with a teammate?

b. Can you describe a negative experience you had with a teammate?

8. Would you consider sport to be an important part of your life?

9. Is being an athlete part of your identity?

10. Is there anything else about your thoughts or experiences in sports that you would like to share?

\section{*turn of audio recorder}

\section{Additional Information (not recorded)}

1. Can you give me a pseudonym to use?

2. Would you like to receive a summary of the findings?

Debriefing - verbal and written debrief. 


\section{Appendix F}

\section{Content Validation Questionnaire (Example)}

Definition of Construct:

\begin{tabular}{|l|l|l|lll|llll|l|}
\hline & Item Measuring & Relevance & Clarity & $\begin{array}{l}\text { Suggestion or } \\
\text { alternate wording }\end{array}$ \\
\hline 1 & & 1 & 2 & 3 & 1 & 2 & 3 & \\
\hline 2 & & 1 & 2 & 3 & 1 & 2 & 3 & \\
\hline 3 & & 1 & 2 & 3 & 1 & 2 & 3 & \\
\hline 4 & & 1 & 2 & 3 & 1 & 2 & 3 & \\
\hline
\end{tabular}

Rating scale:

Relevance: $1=$ not very relevant $\quad 2=$ moderately relevant $\quad 3=$ very relevant

Clarity: $1=$ not very clear $\quad 2=$ moderately clear $\quad 3=$ very clear

1. Are there any aspects of the construct missing from the definition?

2. Are there any aspects of the construct that I failed to include in the items presented?

3. Are there any problems with redundancy in the items?

4. Are there any controversial or problem items?

5. Please provide any additional comments. 
Appendix G

\section{SONA Recruitment Notice}

Study Name: Adolescent Experiences in Organized Sports

Description: This study is looking at adolescent experiences in organized sports and the role that these experiences, along with relationships with coaches and teammates, play in development.

Eligibility Requirements: Participants must be 18 or 19 years old and be playing organized sports (community level or varsity level sports) or have played organized sports in the past 12 months.

Risks: There are no anticipated psychological risks associated with participation. There will be no more physical risk than is consistent with sitting in front of a computer for a one hour period.

Duration and Locale: 60 minutes online.

Compensation: You will receive $0.5 \%$ towards your course (PSYC 1001, 1002, 2001, 2002 or NEUR 2001, 2002).

Researchers: Kate Hill MacEachern (Principal Investigator); Anne Bowker, PhD (Faculty Sponsor)

Email: kate.hillmaceachern@carleton.ca Email: Anne.Bowker@carleton.ca

If you have any ethical concerns with the study, please contact Dr. Bernadette Campbell, Chair, Carleton University Research Ethics Board-B (by phone at 613-520-2600 ext. 4085 or via email at ethics@,carleton.ca).

This study has received clearance by the Carleton University Research Ethics Board B (Clearance \#109554). 
Appendix $\mathrm{H}$

\section{SONA Participant Consent Form}

Title: Adolescent Experiences in Organized Sports

Date of Ethics Clearance: October 4, 2018

Ethics Clearance of the Collection of Data Expires: October 31, 2019

Ethics Clearance: CUREB-B Clearance \# 109554

Purpose of Study: This study aims to understand the role that sport experiences play in positive youth development outcomes for adolescents who participate in organized sports. In addition, this study will look at the relationships between adolescents and their coaches and teammates to better understand the role of these relationships in the sport environment.

The researcher for this study is Kate Hill MacEachern in the Department of Psychology of Carleton University. She is working under the supervision of Anne Bowker, $\mathrm{PhD}$, in the Department of Psychology of Carleton University.

\section{Contact:}

Kate Hill MacEachern

Email: kate.hillmaceachern@,carleton.ca
Anne Bowker, PhD

Anne.Bowker@carleton.ca

Study Description: This study involves a series of questionnaires that will take approximately 60 minutes to complete. The questionnaire will ask you about specific experiences you might have had playing sports and how you feel about playing sports. The questionnaires will also ask you about your experience with coaches and teammates and the attitudes they have towards the sport environment. Finally, you will be asked questions about yourself and how you feel about your abilities in a variety of domains.

Compensation: For your participation in this study, you will receive $0.5 \%$ credits towards your course (PSYC 1001, 1002, 2001, 2002 or NEUR 2001, 2002).

Study Risks: Although it is not anticipated that any questions will cause discomfort, reflecting on yourself can sometimes be uncomfortable. Should you feel discomfort, you may contact the following resource: Carleton University Health and Counseling Services at 613-520-6674

Participation in this study is completely voluntary. Please note that you may choose not to answer questions or end your participation in the study at any time, for any reason without penalty. A withdraw button will be available on each page of the online survey. Please note that you may withdraw from the study at any point up until credit is granted and credit will be granted within 24 hours of the completion of the study.

All research data will be encrypted. Any hard copies of data (such as handwritten notes or USB keys) will be kept in a locked cabinet at Carleton University. Research data will only be accessible by the researcher and the research supervisor. No identifying information will be kept with the data itself to ensure the data remains anonymous. 
Once the project is complete, all anonymized research data will be kept indefinitely and potentially used for other research projects on this same topic. The results of the study may be used in future publications and presentations.

If you would like a summary of the findings once the project is complete, you are invited to contact the researcher to request an electronic copy, which will be provided to you.

The ethics protocol for this project was reviewed by the Carleton University Research Ethics Board, which provided clearance to carry out the research. If you have any ethical concerns with the study, please contact Dr. Bernadette Campbell, Chair, Carleton University Research Ethics Board-B (by phone at 613-520-2600 ext. 4085 or via email at ethics@carleton.ca).

Do you agree to participate in this study?

By clicking yes, you are consenting to participate.

Yes No 
Appendix I

\section{Invitation to participate}

Dear Parent/Guardian,

My name is Kate Hill MacEachern and I am a PhD candidate in the Department of Psychology at Carleton University. I am working on a research project under the supervision of Dr. Anne Bowker entitled Adolescent Experiences in Organized Sports. The purpose of this project is to gain a better understanding of adolescents' experiences in community level or high school organized sports and their role in positive youth development.

Study Requirements: If your child is currently participating in an organized sport or has participated in an organized sport in the past 12 months, I am hoping that you will give permission for your child to participate in this study. This includes team sports, such as hockey, basketball, volleyball, and soccer, etc., and individual sports, such as golf, tennis, dance and martial arts, etc.

Study Description: This study involves a series of questionnaires that will take approximately 60 minutes to complete. First, the questionnaire will ask your child about their experiences playing sports and some of the different things they may have experienced, along with general feelings about playing sports. The questionnaires will then ask about your child's experiences with coaches and teammates. Finally, your child will be asked questions about themselves and how they feel about their abilities in a variety of contexts (e.g. at school or in the community).

Study Risks: I don't anticipate any risks associated with participating in this study. However, participants will be asked to reflect on themselves and that can sometimes cause discomfort. For this reason, resources are made available on the debrief form, should any participant feel a sense of discomfort.

Participation is completely voluntary and has absolutely no impact on student grades.

The ethics protocol for this project has been reviewed by Carleton University Research Ethics Board, which provided clearance to carry out this research (CUREB-B Clearance \#109554). If you have any ethical concerns about this study, contact: Dr. Bernadette Campbell, Chair, Carleton University Research Ethics Board-B (by phone at 613-520-2600 ext. 4085 or via email at ethics@,carleton.ca).

This research has also been approved by the (SCHOOL BOARD) and by the principal of your child's school.

If you consent to your child participating in this study, please complete the parent consent form provided with this letter. Your consideration is very much appreciated. If you have any questions or concerns you would like to discuss, you may contact me at kate.hillmaceachern@,carleton.ca.

Sincerely,

Kate Hill MacEachern 
Appendix J

\section{Consent Form}

Title: Adolescent Experiences in Organized Sports

Date of Ethics Clearance: TBD

Ethics Clearance of the Collection of Data Expires: TBD

Ethics Clearance: CUREB-B Clearance \#109554

I , choose to participate in a study on

experiences in organized sports.

Purpose of Study: This study aims to understand the role that sport experiences play in positive youth development outcomes for adolescents. In addition, this study will look at the relationships between adolescents and their coaches and teammates to better understand how relationships influence experiences and outcomes.

The researcher for this study is Kate Hill MacEachern in the Department of Psychology of Carleton University. She is working under the supervision of Anne Bowker, $\mathrm{PhD}$, in the Department of Psychology of Carleton University.

\section{Contact:}

Kate Hill MacEachern

Email: kate.hillmaceachern@,carleton.ca
Anne Bowker, $\mathrm{PhD}$

Anne.Bowker@carleton.ca

Study Description: This study involves a series of questionnaires that will take approximately 60 minutes to complete. First the questionnaire will ask you about your experiences playing sports and some of the different things you may have experienced, along with your general feelings about playing sports. The questionnaires will then ask you about your experiences with coaches and teammates. Finally, you will be asked questions about yourself and how you feel about your abilities in a variety of contexts (e.g. at school or in your community). Please note that participation is completely voluntary and will not affect student grades in any way.

Compensation: There is no compensation for participating in this study.

Study Risks: It is not anticipated that any questions will cause discomfort. However, answering questions about yourself can sometimes cause discomfort. Should you feel any discomfort, you will be provided with contact information for counseling services available to you.

You have the right to end your participation in the study at any time, for any reason. Any information you have provided will be destroyed immediately, should you choose to withdraw. Participants can withdraw from the study at any point during the study and up to the point that the questionnaire is handed in to the researcher. After this point, questionnaires will not be identifiable.

All research data, including any notes will be encrypted. Any hard copies of data (such as handwritten notes or USB keys) will be kept in a locked cabinet at Carleton University. Research 
data will only be accessible by the researcher and the research supervisor. No identifying information will be kept with the data itself to ensure the data remains anonymous.

Once the project is complete, all anonymized research data will be destroyed. The results of the study may be used in future publications and presentations.

If you would like a summary of the findings once the project is complete, you are invited to contact the researcher to request an electronic copy, which will be provided to you.

The ethics protocol for this project was reviewed by the Carleton University Research Ethics Board, which provided clearance to carry out the research. If you have any ethical concerns with the study, please contact Dr. Bernadette Campbell, Chair, Carleton University Research Ethics Board-B (by phone at 613-520-2600 ext.4085 or via email at ethics@carleton.ca).

Do you agree to participate in this study?

Yes No

Date: 
Appendix K

\section{Debriefing Form}

\section{What are we trying to learn with this research?}

The purpose of this research is to gain a better understanding of the experiences adolescence have in organized sports and how those experiences might promote positive developmental outcomes. Specifically, this study aims to better understand how perceptions of coaches and teammates together with experiences such as goal setting, adversity, and skill development, lead to positive youth development outcomes of competence, confidence, connection, caring, and character. Through this research, we hope to get a better understanding of how and why participation in organized sports is related to positive youth development.

\section{Why is this important to scientists or the general public?}

It is important to understand the types of experiences adolescents are having in sports and what factors lead to a more positive experience so that programs can be appropriately structured to foster positive youth development. This could include the way coaching programs are designed or how sports organizations are structured.

\section{What are our hypotheses and predictions?}

We are expecting that more positive experiences in sports will lead to more positive outcomes. We expect that these experiences, such as getting along with teammates, personal achievements, goal setting, overcoming difficulties (e.g. being cut from a team), and positive relationships with coaches will lead to higher competence, confidence, connection, caring, and character (the 5 Cs of positive youth development).

\section{Where can I learn more?}

To learn more, you can review the following literature:

Holt, N., Neely, K., Slater, L., Camiré, M., Côté, J., Fraser-Thomas, J., ... \& Tamminen, K. (2017). A ground theory of positive youth development through sport based on results from a qualitative meta-study. International Review of Sport and Exercise Psychology, $10,1-49$.

\section{What if I have questions later?}

If you have questions about this study, please feel free to contact to the primary researcher:

Kate Hill MacEachern, MA

kate.hillmaceachern@carleton.ca

If you have concerns or feel distress after responding to any of the questions in the study, please contact any of the following resources:

Distress Centre of Ottawa and Region at: 613-238-3311 (http://www.dcottawa.on.ca). 
If you have any ethical concerns with this study, please contact Dr. Bernadette Campbell, Chair, Carleton University Research Ethics Board-B (by phone at 613-520-2600 ext. 4085 or via email at ethics@,carleton.ca).

Thank you for participating!!

This research was cleared by Carleton University Research Board B Clearance \#109554 
Appendix L

\section{Background Information Questionnaire}

\section{Background Information}

1. Age:

2. Gender: Male Female

Other Prefer not to specify

3. What is the highest level of education obtained by your mother?
a. Some high school
b. High school graduate
c. Some college/university
d. College graduate
e. University degree
f. Some postgraduate
g. Postgraduate degree

4. Would you consider where you live to be urban, suburban, or rural?
a. Urban
b. Suburban
c. Rural

The following questions ask about your participation in community level organized sports. Community level organized sports take place with a league or organization. There are organized times for practices 


\section{and games/matches/competitions. For these questions, do not include} high school sports (you will be asked about these next).

1. How many years have you been playing an organized sport?

2. Please indicate all of the community level organized sports you have played:

Hockey

Swimming

Ringette

Soccer

Rugby

Ultimate Frisbee

Football

Martial Arts

Volleyball

Horseback Riding
Water Polo

Curling

Gymnastics

Cheerleading

Cricket

Figure Skating

Softball/Fastball

Roller Derby

Skiing

Dance
Basketball

Baseball

Track and Field

Rowing

Field Hockey

Badminton

Lacrosse

Wrestling

Other:

3. What organized sport(s) have you played in the past 12 months?

Hockey

Swimming

Ringette

Soccer

Rugby

Ultimate Frisbee

Football

Martial Arts
Water Polo

Curling

Gymnastics

Cheerleading

Cricket

Figure Skating

Softball/Fastball

Roller Derby

Skiing
Basketball

Baseball

Track and Field

Rowing

Field Hockey

Badminton

Lacrosse

Wrestling 
Volleyball Dance

Horseback Riding

Other (please

specify):

4. Are you currently playing an organized sport(s)?

YES NO

a. If Yes, what?

5. In the past 12 months, how many hours per week do you typically commit to playing organized sports? You can include organized training sessions and any work you do on your own time in the training category.

Games/Matches/Competitions ___ hrs/week

Practices __ hrs/week

Training _ _ h hrs/week

6. In the sport (or sports) you are currently participating in, do you play at a recreational (e.g., house league, non-competitive) or competitive (e.g., rep) level (if not currently in an organized sport, select the sport you have most recently played)?

Recreational/house league

Competitive/rep

7. From when you first started playing organized sports, how many years have you played on recreational and/or competitive teams?

a. Recreational___ years

b. Competitive___ years 
The following questions ask about your high school sport participation. Please only include high school sports that involved try-outs and competitions against other schools. Do not include intramural or gym class sports.

8. Are you currently a member of a high school sports team?

$$
\text { YES NO }
$$

If yes, what?

a. How many years have you played on this high school team?

9. Have you played any other high school sports in the past 12 months? YES NO

a. If yes, what?

10. How many hours per week do you commit to playing your high school sport? You can include organized training sessions and any work you do on your own time in the training category.

Games/Matches/Competitions hrs/week

Practices hrs/week

Training hrs/week 
11. Do you participate in any other extracurricular activities in the community or at school? (select all that apply)
a. Music (e.g., play an instrument, play in the school band)
b. Art
c. Drama
d. Volunteering
e. Youth group (e.g., Boy Scouts/Girl Guides)
f. Church group

When answering the following sets of questions, please think about the organized sport you are currently participating in. This can be a high school team or community level sport. If you are not currently participating in an organized sport, please think about the sport you have most recently participated in (e.g., if it is the winter and you played soccer in the summer, think about soccer). If you are participating in more than one sport, please think about the one that you would consider your favourite.

Please indicate the organized sport you will be thinking about when answering the following questions:

Is this sport high school or community level? (please circle)

$$
\text { High School Community }
$$

Would you consider this sport recreational (e.g., house, non-competitive) or competitive? (please circle) 


\section{Recreational/house league Competitive}

Is this sport your favourite sport? (please circle)

Yes No 


\section{Appendix M}

\section{Experience in Sport Scale for Youth}

When answering the following questions, think about your experiences playing organized sports and how closely the items describe you and your experiences.

$\begin{array}{ccccc}\text { Never } & \text { Rarely } & \text { Occasionally } & \text { Frequently } & \text { Always } \\ 1 & 2 & 3 & 4 & 5\end{array}$

I set my own goals.

I have achieved goals.

I set goals with my group/team.

I have worked hard to achieve a goal.

I set long term goals.

I set short term goals.

I have specific plans/steps to achieve my goals.

I have told others about my goals.

I regularly reviewed my goals.

I have faced challenges (e.g., cut from a

team, suffered an injury, had to sit out games, etc.)

$\begin{array}{lllllll}\text { I have lost an important game/competition. } & 1 & 2 & 3 & 4 & 5\end{array}$

I have struggled to learn a new skill.

I have played on a team where I have not gotten along with some people.

I have made a mistake that cost me or my team. $\begin{array}{lllll}1 & 2 & 3 & 4 & 5\end{array}$

$\begin{array}{lllll}1 & 2 & 3 & 4 & 5\end{array}$

$\begin{array}{lllll}1 & 2 & 3 & 4 & 5\end{array}$

$\begin{array}{lllll}1 & 2 & 3 & 4 & 5\end{array}$

$\begin{array}{lllll}1 & 2 & 3 & 4 & 5\end{array}$

$\begin{array}{lllll}1 & 2 & 3 & 4 & 5\end{array}$

$\begin{array}{lllll}1 & 2 & 3 & 4 & 5\end{array}$

$\begin{array}{lllll}1 & 2 & 3 & 4 & 5\end{array}$

$\begin{array}{lllll}1 & 2 & 3 & 4 & 5\end{array}$

$\begin{array}{lllll}1 & 2 & 3 & 4 & 5\end{array}$

$\begin{array}{lllll}1 & 2 & 3 & 4 & 5\end{array}$

$\begin{array}{lllll}1 & 2 & 3 & 4 & 5\end{array}$

$\begin{array}{lllll}1 & 2 & 3 & 4 & 5\end{array}$ 
$\begin{array}{llllllll}\text { I have had the opportunity to develop new } & 1 & 2 & 3 & 4 & 5\end{array}$ skills.

$\begin{array}{lllllll}\text { I have worked hard to develop a specific } & 1 & 2 & 3 & 4 & 5\end{array}$ skill.

$\begin{array}{llllllll}\text { I have had the opportunity to develop a } & 1 & 2 & 3 & 4 & 5\end{array}$ wide range of physical skills.

$\begin{array}{lllllll}\text { I had a lot of help from others to improve } & 1 & 2 & 3 & 4 & 5\end{array}$ skills.

$\begin{array}{lllllll}\text { I have worked on skills on my own time. } & 1 & 2 & 3 & 4 & 5\end{array}$ 
Appendix N

Peer Motivational Climate in Youth Sport Questionnaire - PeerMCYSQ

$1=$ strongly disagree

$7=$ strongly agree

On this team, most athletes...

1. Help each other improve.

2. Encourage each other to outplay their teammates.

3. Offer to help their teammates develop new skills.

4. Care more about the opinion of the most able teammates.

5. Make their teammates feel valued.

6. Work together to improve the skills they don't do well.

7. Make negative comments that put their teammates down.

8. Try to do better than their teammates.

9. Criticize their teammates when they make mistakes.

10. Teach their teammates new things.

11. Encourage their teammates to try their hardest

12. Look pleased when they do better than their teammates.

13. Make their teammates feel accepted.

14. Want to be with the most able teammates.

15. Praise their teammates to try their hardest.

16. Complain when the team doesn't win.

17. Are pleased when their teammates try hard.

18. Care about everyone's opinion. 
19. Set an example on giving forth maximum effort.

20. Laugh at their teammates when they make mistakes.

21. Encourage their teammates to keep trying after they make a mistake. 
Appendix $\mathrm{O}$

Coach Climate Questionnaire - MCSYS

Here are some statements about what your current team is like. Please read each one and circle the number that is most correct for you and your team. If there is more than one coach on your team, the questions are about the coach that you spend most of your time with.

$\begin{array}{lcccc}1 & 2 & 3 & 4 & 5 \\ \text { Not at all true } & & \text { Somewhat true } & & \text { Very true }\end{array}$

1. Winning games/competitions is the most important thing for the coach.
1
2
3
4
5

2. The coach makes players feel good when they improve a skill.

$\begin{array}{lllll}1 & 2 & 3 & 4 & 5\end{array}$

3. The coach spends less time with the players who weren't as good.

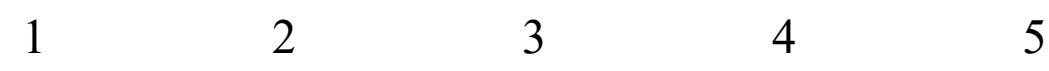

4. The coach encourages us to learn new skills.

$\begin{array}{lllll}1 & 2 & 3 & 4 & 5\end{array}$

5. The coach told us which players on the team were the best.
1
2
3
4
5

6. The coach told players to help each other get better.
1
2
3
4
5

7. The coach told us that trying our best was the most important thing. 
8. The coach pays most attention to the best players.

$\begin{array}{llllll}1 & 2 & 3 & 4 & 5\end{array}$

9. The coach said that teammates should help each other improve their skills.
1
2
3
4
5

10. Players were taken out of games/competitions if they made a mistake.

$\begin{array}{llllll}1 & 2 & 3 & 4 & 5\end{array}$

11. The coach said that all of us were important to the team's success.

$\begin{array}{llllll}1 & 2 & 3 & 4 & 5\end{array}$

12. The coach told us to try to be better than our teammates.

$\begin{array}{llllll}1 & 2 & 3 & 4 & 5\end{array}$


Appendix $\mathrm{P}$

\title{
PYD Short Form for Older Adolescents (Geldhof et al., 2014)
}

\author{
PYD Short Form: Older Adolescents (34 items) \\ PYD Very Short Form items indicated in red (17 items)
}

\begin{tabular}{|c|c|c|c|c|c|c|c|c|}
\hline \multicolumn{9}{|c|}{ 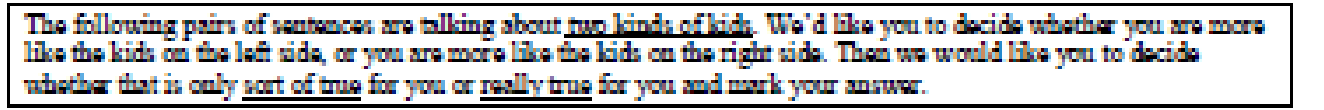 } \\
\hline \multicolumn{9}{|c|}{ FILL IN ONLY ONE CIRCLF FOR EACH PAIR OF SENTENCES. } \\
\hline \begin{tabular}{|r|r|} 
& $R$ \\
& $T$ \\
& fo \\
\end{tabular} & $\begin{array}{l}\text { Really } \\
\text { True } \\
\text { for } m e\end{array}$ & $\begin{array}{l}\text { Sort of } \\
\text { True } \\
\text { ior me }\end{array}$ & & & & & $\begin{array}{l}\text { Sort of } \\
\text { True } \\
\text { for me }\end{array}$ & $\begin{array}{l}\text { Really } \\
\text { Trae } \\
\text { for } m e x\end{array}$ \\
\hline \multirow[t]{2}{*}{ (a) } & 0 & o & $\begin{array}{l}\text { Seme kids would nather } \\
\text { play cutdoers in their } \\
\text { pare time }\end{array}$ & BUT & Ot & $\begin{array}{l}\text { ar kids would rather } \\
\text { ch T.V. }\end{array}$ & 0 & o \\
\hline & $\begin{array}{l}\text { Really } \\
\text { True } \\
\text { for we } \\
{[4]}\end{array}$ & $\begin{array}{c}\text { Sort of } \\
\text { True } \\
\text { for me } \\
{[3]}\end{array}$ & & & & $\begin{array}{l}\text { Sort of } \\
\text { True } \\
\text { for me } \\
{[2]}\end{array}$ & \begin{tabular}{l|c} 
Reslly \\
True \\
for me \\
11
\end{tabular} \\
\hline 1. harrol & 0 & 0 & \multicolumn{2}{|l|}{ 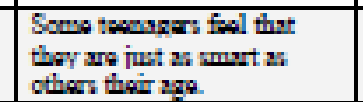 } & BUT & $\begin{array}{l}\text { Other teensages aren't wo } \\
\text { wre and wouder if ther are } \\
\text { as anst. }\end{array}$ & 0 & 0 \\
\hline 2. hartll & o & $\overline{0}$ & \multicolumn{2}{|l|}{$\begin{array}{l}\text { Sown tecesgas burv a lot } \\
\text { of frieuds. }\end{array}$} & BUT & $\begin{array}{l}\text { Other toensgers don't thave } \\
\text { very mavy frieds. }\end{array}$ & 0 & o \\
\hline 3. hartl2 & o & 0 & \multicolumn{2}{|l|}{$\begin{array}{l}\text { Souve teowages thint they } \\
\text { could do woll at juat about } \\
\text { any now zthletic activity. }\end{array}$} & BUT & $\begin{array}{l}\text { Other toensigers are afraid } \\
\text { tery might pot do well at a } \\
\text { Dow athletic activity. }\end{array}$ & 0 & 0 \\
\hline 4. hart19 & 0 & 0 & \multicolumn{2}{|l|}{$\begin{array}{l}\text { Scuve teeengar do vary } \\
\text { well at thair class work. }\end{array}$} & BUT & $\begin{array}{l}\text { Other trensagen don tdo } \\
\text { very woll at thair class } \\
\text { work. }\end{array}$ & 8 & 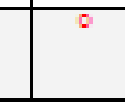 \\
\hline 5. hart21 & $\overline{0}$ & $\overline{0}$ & \multicolumn{2}{|l|}{ 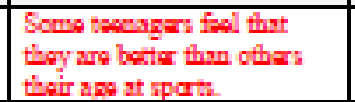 } & BUT & $\begin{array}{l}\text { Other toensgers don't foel } \\
\text { toy camplay as wall. }\end{array}$ & $\overline{0}$ & o \\
\hline 6. hart27 & 0 & 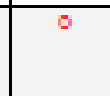 & 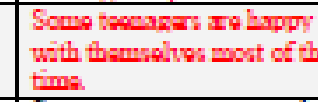 & & BUT & 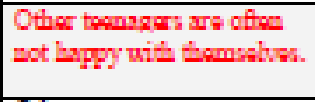 & 0 & 0 \\
\hline 7. hare29 & 0 & 0 & $\begin{array}{l}\text { Sowse treosgas re populs } \\
\text { with others thair ans. }\end{array}$ & & BUT & $\begin{array}{l}\text { Other toensigen are not } \\
\text { very populyr. }\end{array}$ & 0 & 0 \\
\hline 8. harkI & 0 & 0 & $\begin{array}{l}\text { Scowe teenagar fint that } \\
\text { thoy are good looking. }\end{array}$ & & BUT & $\begin{array}{l}\text { Cther toenagers think fart } \\
\text { they are not very good } \\
\text { looking. }\end{array}$ & 0 & 0 \\
\hline $\begin{array}{c}\text { 9. horrit } \\
\text { reverse } \\
\text { code }\end{array}$ & 8 & $\overline{0}$ & $\begin{array}{l}\text { Sowo tocongers do fings } \\
\text { they know they houldw't } \\
\text { do. }\end{array}$ & & BuT & $\begin{array}{l}\text { Other toenagers hardly } \\
\text { over do things they loow } \\
\text { ther shoulin't do. } \\
\end{array}$ & $\overline{0}$ & $\overline{0}$ \\
\hline 10. hart40 & 0 & 0 & $\begin{array}{l}\text { Sows tecengars really like } \\
\text { their locks. }\end{array}$ & & BUT & $\begin{array}{l}\text { Other trensegrs with thoy } \\
\text { lockod difforest. }\end{array}$ & 0 & 0 \\
\hline 11. bart43 & 0 & 0 & $\begin{array}{l}\text { Sowo teenagas wanlly ac } \\
\text { the uny thoy know they ar } \\
\text { surpored to. }\end{array}$ & & BUT & $\begin{array}{l}\text { Other toenegers offen den't } \\
\text { act the way they are } \\
\text { sagored to. }\end{array}$ & 0 & $\circ$ \\
\hline 12. bart45 & 0 & 0 & $\begin{array}{l}\text { Sowe treosars are very } \\
\text { happy being the way they } \\
\text { are. }\end{array}$ & & BUT & $\begin{array}{l}\text { Other toensags with they } \\
\text { were different. }\end{array}$ & 0 & 0 \\
\hline
\end{tabular}


How much do you agree or disagree with the following?

\begin{tabular}{|c|l|c|c|c|}
\hline $\begin{array}{c}\text { Strongly } \\
\text { disagree } \\
(1)\end{array}$ & $\begin{array}{l}\text { Disagree } \\
(2)\end{array}$ & $\begin{array}{c}\text { Not } \\
\text { sure } \\
(3)\end{array}$ & $\begin{array}{c}\text { Agree } \\
(4)\end{array}$ & $\begin{array}{c}\text { Strongly } \\
\text { Agree } \\
(5)\end{array}$ \\
\hline
\end{tabular}

13. All in all, I am glad I am me.

$\begin{array}{lllll}1 & 2 & 3 & 4 & 5\end{array}$

14. When I am an adult, I'm sure I will have a good life.

$\begin{array}{lllll}1 & 2 & 3 & 4 & 5\end{array}$

How important is each of the following to you in your life?

\begin{tabular}{|c|l|c|c|c|}
\hline Not & Somewh & No & Quite & Extremel \\
Importa & at & $\mathrm{t}$ & Importa & $\mathrm{y}$ \\
$\mathrm{nt}$ & importan & sur & $\mathrm{nt}$ & Importan \\
$(1)$ & $\mathrm{t}(2)$ & $\mathrm{e}$ & $(4)$ & $\mathrm{t}(5)$ \\
\hline
\end{tabular}

15. Helping to make the world a better place to life in.

$\begin{array}{lllll}1 & 2 & 3 & 4 & 5\end{array}$

16. Giving time and money to make life better for other people.

$\begin{array}{lllll}1 & 2 & 3 & 4 & 5\end{array}$

17. Doing what I believe is right even if my friends make fun of me.

$\begin{array}{lllll}1 & 2 & 3 & 4 & 5\end{array}$

18. Accepting responsibility for my actions when I make a mistake.

$\begin{array}{lllll}1 & 2 & 3 & 4 & 5\end{array}$

Think about the people who know you well. How do you think they would rate you on each of these? 


\begin{tabular}{|c|l|c|c|c|}
\hline $\begin{array}{c}\text { Not at } \\
\text { all like } \\
\text { me }\end{array}$ & A little & $\begin{array}{c}\text { Somewhat } \\
\text { like me }\end{array}$ & $\begin{array}{c}\text { Quite } \\
\text { like }\end{array}$ & $\begin{array}{c}\text { Very } \\
\text { much }\end{array}$ \\
$(1)$ & me & $(3)$ & me(4) & like me \\
& $(2)$ & & & $(5)$ \\
\hline
\end{tabular}

19. Knowing a lot about people of other races. $\quad \begin{array}{llllll}1 & 2 & 3 & 4 & 5\end{array}$

20. Enjoying being with people who are of a different race than I am.

$\begin{array}{lllll}1 & 2 & 3 & 4 & 5\end{array}$

How well do each of these statements describe you?

\begin{tabular}{|c|l|l|l|c|}
\hline $\begin{array}{c}\text { Not well } \\
(1)\end{array}$ & $(2)$ & $(3)$ & $(4)$ & $\begin{array}{c}\text { Very } \\
\text { well (5) }\end{array}$ \\
\hline
\end{tabular}

21. When I see someone being taken advantage of, I want to help them.

$\begin{array}{lllll}1 & 2 & 3 & 4 & 5\end{array}$

22. It bothers me when bad things happen to any person.

$\begin{array}{lllll}1 & 2 & 3 & 4 & 5\end{array}$

23. I feel sorry for other people who don't have what I have.

$\begin{array}{lllll}1 & 2 & 3 & 4 & 5\end{array}$

24. When I see someone being picked on, I feel sorry for them.

$\begin{array}{lllll}1 & 2 & 3 & 4 & 5\end{array}$

25. It makes me sad to see a person who doesn't have friends.

$\begin{array}{lllll}1 & 2 & 3 & 4 & 5\end{array}$

26. When I see another person who is hurt or upset, I feel sorry for them.

$\begin{array}{lllll}1 & 2 & 3 & 4 & 5\end{array}$


How much do you agree or disagree with the following?

\begin{tabular}{|c|l|c|c|c|}
\hline $\begin{array}{c}\text { Strongly } \\
\text { disagree } \\
(1)\end{array}$ & $\begin{array}{l}\text { Disagree } \\
(2)\end{array}$ & $\begin{array}{c}\text { Not } \\
\text { sure } \\
(3)\end{array}$ & $\begin{array}{c}\text { Agree } \\
(4)\end{array}$ & $\begin{array}{c}\text { Strongly } \\
\text { Agree } \\
(5)\end{array}$ \\
\hline
\end{tabular}

27. I get a lot of encouragement at my school. $\quad \begin{array}{lllllll}1 & 2 & 3 & 4 & 5\end{array}$

28. Teachers at school push me to be the best I can be. $\quad \begin{array}{lllll}1 & 2 & 3 & 4\end{array}$ 5

29. I have lots of good conversations with my parents. $\begin{array}{lllll}1 & 2 & 3 & 4\end{array}$ 5

30. In my family I feel useful and important. 5

$\begin{array}{llll}1 & 2 & 3 & 4\end{array}$

31. Adults in my town or city make me feel important. $\quad \begin{array}{lllll}1 & 2 & 3 & 4\end{array}$ 5

32. Adilts in my town or city listen to what I have to say. $\begin{array}{llllll}1 & 2 & 3 & 4\end{array}$ 5

How true is each of these statements for you?

\begin{tabular}{|c|c|c|c|c|}
\hline $\begin{array}{c}\text { Alway } \\
\text { s True } \\
\text { (1) }\end{array}$ & $\begin{array}{l}\text { Usuall } \\
\text { y True } \\
(2)\end{array}$ & $\begin{array}{l}\text { Sometime } \\
\text { s } \\
\text { True (3) }\end{array}$ & $\begin{array}{c}\text { Seldo } \\
\text { m True } \\
(4)\end{array}$ & $\begin{array}{c}\text { Almos } \\
\text { t never } \\
\text { true or } \\
\text { never } \\
\text { true } \\
(5)\end{array}$ \\
\hline
\end{tabular}

33. I feel my friends are good friends. $\quad \begin{array}{llllll}1 & 2 & 3 & 4 & 5\end{array}$

34. My friends care about me. $\quad \begin{array}{llllll}2 & 2 & 3 & 4 & 5\end{array}$


